\title{
Population Studies Of The Native Eastern Oyster, Crassostrea Virginica, (Gmelin, 1791) In The James River, Virginia, Usa
}

Roger L. Mann

Virginia Institute of Marine Science

Melissa Southworth

Virginia Institute of Marine Science

Juliana M. Harding

Virginia Institute of Marine Science

James A. Wesson

Follow this and additional works at: https://scholarworks.wm.edu/vimsarticles

Part of the Marine Biology Commons

\section{Recommended Citation}

Mann, Roger L.; Southworth, Melissa; Harding, Juliana M.; and Wesson, James A., "Population Studies Of The Native Eastern Oyster, Crassostrea Virginica, (Gmelin, 1791) In The James River, Virginia, Usa" (2009). VIMS Articles. 405.

https://scholarworks.wm.edu/vimsarticles/405

This Article is brought to you for free and open access by the Virginia Institute of Marine Science at W\&M ScholarWorks. It has been accepted for inclusion in VIMS Articles by an authorized administrator of W\&M ScholarWorks. For more information, please contact scholarworks@wm.edu. 


\title{
POPULATION STUDIES OF THE NATIVE EASTERN OYSTER, CRASSOSTREA VIRGINICA, (GMELIN, 1791) IN THE JAMES RIVER, VIRGINIA, USA
}

\author{
ROGER MANN, ${ }^{1 *}$ MELISSA SOUTHWORTH, ${ }^{1}$ JULIANA M. HARDING ${ }^{1}$ \\ AND JAMES A. WESSON ${ }^{2}$ \\ ${ }^{1}$ Department of Fisheries Science, Virginia Institute of Marine Science, College of William and Mary, \\ P.O. Box 1346, Gloucester Point, Virginia 23062; ${ }^{2}$ Virginia Marine Resources Commission, \\ 2600 Washington Ave, Newport News, Virginia 23607
}

\begin{abstract}
We describe oyster population trends in the James River, VA from 1993 through 2006 using quantitative fishery independent survey data collected using a stratified random design. The 23 reefs contained in the study area cover a total of 2.41 $\times 10^{7} \mathrm{~m}^{2}$ and vary in individual size from $1.26 \times 10^{4} \mathrm{~m}^{2}$ to $4.98 \times 10^{6} \mathrm{~m}^{2}$. There is a marked pattern in density of oysters among the reefs: during the study period a small group of reefs comprising $5.4 \%$ of the total area consistently contained between 25.7 and $55.5 \%$ by number and 35.8 and $54.8 \%$ by biomass of the total oyster population. The highest density reefs exhibit, with very few exceptions, mean densities well in excess of 200 oysters $\mathrm{m}^{-2}$, typically between 300 and $500 \mathrm{~m}^{-2}$, with a single maximum value of 773 oysters $\mathrm{m}^{-2}$ in 2002 coincident with the highest annual recruitment observed during the study period. Recruitment events were usually followed by very high mortality with very small percentages of the population reaching ages $\geq 3 \mathrm{y}$ of age. A strong stockrecruit relationship is absent; rather population demographics appear to be dominated by periodic high recruitment events. Biomass maxima tended to lag one to two years after recruitment maxima. Standing stock for the total system varied between 1.07 $\times 10^{8} \mathrm{~g}$ and $3.31 \times 10^{8} \mathrm{~g}(107$ and 331 metric tonnes) in 2003 and 2005 , respectively as the 2002 recruits grew and suffered mortality. Age-at-length relationships were estimated from demographics: using a July 1 birth date and a November 1 survey date giving lengths of $37.3 \mathrm{~mm}$ at $0.33 \mathrm{y}, 58.9 \mathrm{~mm}$ at $1.33 \mathrm{y}, 80.5 \mathrm{~mm}$ at $2.33 \mathrm{y}, 102.1 \mathrm{~mm}$ at $3.33 \mathrm{y}$ and $123.7 \mathrm{~mm}$ at $4.33 \mathrm{y}$ Length demographics were recast as age demographics to estimate annual proportional mortality. Mean proportional mortality values for age 1 oysters range from a low of $0.2-0.4$ to a high in excess of 0.7 . Age 2 mean proportional mortality values range from a low of 0.41 to a high exceeding 0.75 . The proportional mortality for age 3 and 4 y olds generally exceeded mean values of 0.6 with highest values approaching 0.95 . In all cases, these values exceeded mortality estimates calculated using traditional box count methods by a considerable margin. The ability to accurately estimate age specific mortality allows the construction of shell (habitat) budgets for the individual reef systems. Shell half-life loss rate estimates in the most productive reefs is between 2 and $3 \mathrm{y}$, and the population is maintained by the continual and extraordinary recruitment in the face of high mortality - the latter driven by disease (predominantly Perkinsus marinus) epizootics. The shell resource, even on the most productive reefs, is modest, equivalent to little more than a monolayer several centimeters thick. Individual reefs demonstrate remarkable stability as either high shell density + high population density associations (high:high) or low shell density + low population density associations (low:low), even in the face of temporal population and demographic fluctuations associated with disease related mortality. The probability of manipulating either shell and/or live oyster density to effect the transition of a low:low reef to a high:high reef is considered bleak in the face of extant recruitment and mortality patterns. The primary impediment to population expansion or rebuilding is high and uncontrolled mortality rather than a lack of recruitment. Given the large numbers of oysters in low salinity refugia that have the ability to continually contribute to the larval pool, active selection against disease susceptible oysters on a system wide basis is unlikely.
\end{abstract}

KEY WORDS: Oyster, Eastern oyster, Crassostrea virginica, population demographics, mortality, recruitment, James River, Virginia

\section{INTRODUCTION}

The Eastern oyster, Crassostrea virginica (Gmelin, 1791), has long played an important ecological role in the Chesapeake Bay and its tributaries. As the Bay emerged approximately 10,000 y ago with rising sea level, oysters invaded this ephemeral geological feature and formed a mosaic of reef structures that would become keystone features in both the habitat and trophic system of the Bay community (Hargis 1999). Chesapeake Bay oysters were harvested by native Americans prior to colonial settlement (1607) and had become a central component of the regional fishery economy by 1840 . The need for oyster resource management in the Chesapeake Bay was recognized in the late 19th century (Ingersoll 1881, Brooks 1891, Moore 1910). Under the direction of Lt. J.B. Baylor, a survey of bottom areas in Virginia where oysters occurred naturally was completed in 1896 (Baylor 1896) and was later updated by Haven et al.

*Corresponding author. E-mail: rmann@vims.edu
(1981). These areas, known as the Baylor Survey Grounds or Public Oyster Grounds of Virginia, are currently under the management of the Virginia Marine Resources Commission (VMRC). With the continuing decline of Virginia's oyster population and its current precarious state (Hargis \& Haven 1988, 1995, 1999), native oyster management efforts since the early 1990s have increasingly focused on population rebuilding. These efforts have taken many forms including construction of intertidal oyster reefs (Mann et al. 1996, Wesson et al. 1999, Bartol \& Mann 1997, Bartol \& Mann 1999a, Bartol \& Mann1999b, Luckenbach et al. 1999, Mann 2000), broodstock enhancement (Southworth \& Mann 1998), shell plants or the placement of thin veneers of clean shell on the bottom (Southworth et al. 2000a) or a combination of any of the above. To date these efforts have met with limited success and the future for improvement is bleak (Mann \& Powell 2007).

In a scenario where essential habitat (Benaka 1999) is not limiting, rebuilding of a fishery resource is typically approached as an exercise in optimizing recruitment while limiting exploitation 
(fishing mortality). Oysters are unusual in that they create their own habitat. Thus, rebuilding populations is a matter of understanding the dynamics of both the population and the habitat in concert (Powell et al. 2006, Powell \& Klinck 2007, Mann \& Powell 2007). Within the Chesapeake Bay, the abundance and biomass of most extant oyster populations has been decimated by the combined impacts of exploitation, disease, and habitat loss. The James River, Virginia oyster habitat is a microcosm of the Chesapeake Bay in general in that it encompasses a salinity gradient with lower salinity locations subject to spring freshet events and higher salinity locations characterized by disease epizootics in low river flow (high salinity) years (Andrews 1984, 1988, Burreson \& Ragone-Calvo 1996). The Burwell Bay oyster population in the James River (Fig. 1) has exhibited relative stability in both abundance and biomass over decadal time scales (Haven et al. 1981, Haven \& Whitcomb 1983, Mann \& Evans 1998). This region is partially insulated from disease by low seasonal salinities and also sustains a modest commercial fishery. As such, a basic understanding of the population and habitat dynamics of the oyster population from the mouth of the Warwick River to approximately Mulberry Point, including the Burwell Bay system (Fig. 1), is a valuable model for consideration in defining both the limitations and opportunities, if any exist, for rebuilding of the entire Chesapeake Bay oyster population.

Fishery independent estimates of oyster abundance are essential for estimation of standing stock and fundamental to the design and evaluation of resource management and rebuilding protocols as well as establishment of commercial fishery catch quotas. Both patent tongs and dredges are commonly used to examine oyster populations. However, only the former provide good quantitative estimates (Chai et al. 1992, Powell et al. 2002, Mann et al. 2004). Dredges provide semiquantitative data, have been used with consistency over extended periods (decades) in Virginia, and describe population trends. Absolute quantification of dredge data is difficult in that dredges accumulate organisms as they move over the bottom, may not sample with constancy throughout a single dredge haul, and may fill before completion of the haul thereby providing biased sampling (Powell et al. 2002, Mann et al. 2004).

Patent tongs provide quantitative data as per tong estimates of oysters by size class and shell by volume (Mann et al. 2004). A collaborative effort between the Virginia Institute of Marine Science (VIMS) and VMRC began annual stock assessment surveys of Virginia's Public Oyster Grounds using hydraulic patent tongs in 1993. Initial surveys were focused primarily in tributaries where there was commercial activity (Mann \& Evans 1998, 2004), with other tributaries and areas of interest being added in later years based on restoration efforts (Southworth et al. 2000a). The James River historically provided seed (submarket sized) oysters for grow-out in other rivers as well as oysters for market (Haven et al. 1981) and remains as the only river of note in Virginia that has supported and continues to support a commercial public fishery. Modest harvests are

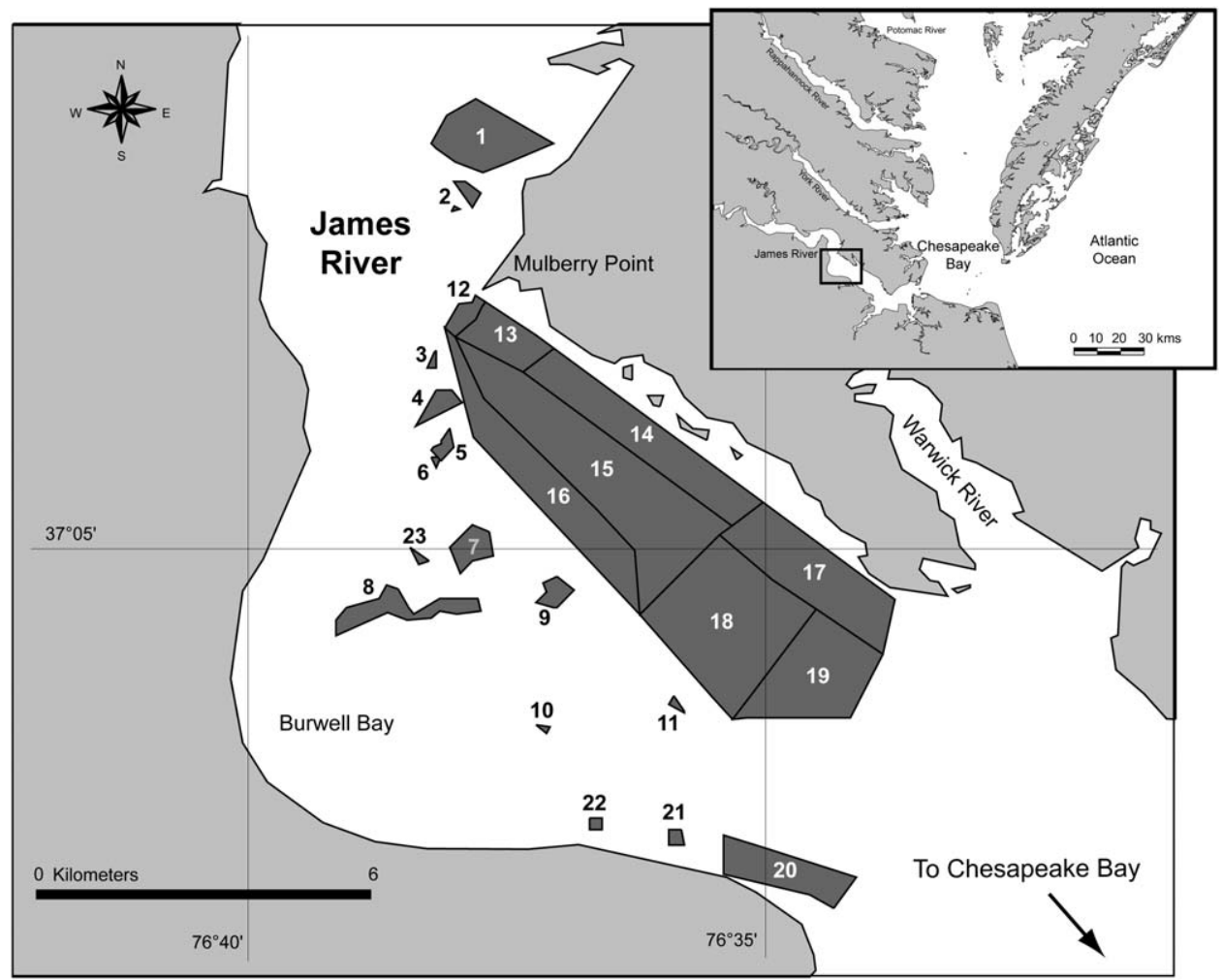

Figure 1. Map showing the locations of the 23 reefs sampled during the patent tong survey in the James River, VA on an annual basis 1993 to 2006 . (1) Upper Deep Water Shoal, (2) Lower Deep Water Shoal, (3) Upper Horsehead, (4) Middle Horsehead, (5) Lower Horsehead, (6) Moon Rock, (7) V-Rock, (8) Point of Shoal, (9) Cross Rock, (10) Shanty Rock, (11) Dry Lumps, (12) Mulberry Point, (13) Swash, (14) Upper Jail Island, (15) Swash and Mud Slough, (16) Offshore Swash, (17) Lower Jail Island, (18) Offshore Jail Island, (19) Wreck Shoal, (20) Day's Point, (21) Hotel Rock, (22) Snyder's Rock, and (23) Triangle Rock. The numbering system will be used throughout the text and figures. 
periodically taken from other rivers, but these are both inconsistent and small in volume compared with the James River harvests. The circulation of the river is well documented, from the contributions of Pritchard $(1952,1953)$ as well as more recent three-dimensional models (Hamrick 1992, Shen \& Kuo 1999, Shen et al. 1999). The relationship of circulation to recruitment processes has been extensively examined (Pritchard 1952, 1953, Wood \& Hargis 1971, Haven \& Fritz 1985, Mann 1988, Ruzecki \& Hargis 1989, Mann \& Evans 1998, Shen et al. 1999) with a collective agreement that the river system is self-recruiting with assistance from both gyre and frontal features. We consider the James River oyster population as a single stock with modest larval losses to emigration, but effectively no immigration of larvae from other sub estuaries of the Chesapeake Bay.

We describe oyster population trends in the James River from the mouth of the Warwick River to just above Mulberry Point from 1993 through 2006; the relationships between individual growth, stock size, mortality, recruitment, and habitat; and relate these observations to inter and intra-annual meteorological events, disease epizootics and fishery practices over the study time period.

\section{MATERIALS AND METHODS}

Oysters for the current study were collected during the fall (October through November) from natural oyster reefs in the James River (Fig. 1) from 1993 through 2006. A quantitative sampling program was used at all reefs using a stratified random grid with documented oyster reefs (bars) forming the strata. A list of reefs sampled is given in the legend of Figure 1. Nineteen reefs were sampled in 1993, and twenty-three were sampled from 1994 through 2006. In most cases, these reefs adhere to the locations and names used by Baylor and subsequently resurveyed by Haven et al. (1981) in the late 1970s, and described in Mann \& Evans (1998).

\section{Temperature and Salinity Data}

Water temperature data were collected on a weekly basis from June through October at eight sites (reefs 1, 4, 8, 11, 13, 19, 20 and 21, Fig. 1) throughout the study period. From 19932004, water samples were collected approximately $0.5 \mathrm{~m}$ off the bottom and temperature was measured with an alcohol thermometer within 5 min of water sample collection. Beginning in 2005, water temperature was measured with a hand-held digital probe (YSI 85) suspended $0.5 \mathrm{~m}$ from the bottom. Data collected within one week were consistently with a range of $1^{\circ} \mathrm{C}$ and were averaged across all sites. We use temperature data from the York River (VIMS Ferry Pier data, Gloucester Point VA, Lat $37^{\circ} 14^{\prime} 47^{\prime \prime}$, Long $76^{\circ} 30^{\prime} 23^{\prime \prime}$ ) averaged on a weekly basis as a surrogate in the months (October through May) where data are lacking from the James River. Comprehensive in situ salinity data from the James River are lacking for the study period. It has been shown that river flow is a good surrogate for salinity (Mann \& Evans 1998). Using the salinity flow relationship from Mann and Evans (1998), we converted river flow data from the United States Geological Survey (http:// nwis.waterdata.usgs.gov) to estimate monthly salinities at reefs 1 and 19 (Fig. 1) during the study period. We note an error in the published relationship in Mann and Evans (1998) in that the units should be cubic feet per second, not cubic feet per minute.

\section{Oyster Field Collections}

Oysters were collected from the 43-ft long VMRC vessel J.B. Baylor with a hydraulic patent tong. The open dimensions of the tong were such that it sampled one square meter of bottom. Upon retrieval of each sample (=patent tong grab), oysters were counted and measured ( $\mathrm{mm}$ ), and the volume of shell material (L) recorded. The recorded dimension on each oyster was the longest from the hinge to the shell growth margin. This is correctly termed shell height, although commonly described as shell length in most literature. We adopt the common convention and refer to shell length (SL) in the subsequent text. A count of the number of oysters per tong was made in all years sampled (1993 through 2006). Prior to 1998 a representative subsample $(n>100)$ of oysters was pooled across individual samples for a given reef, measured, and classified into $5 \mathrm{~mm}$ size bins. From 1998 to 2003, for each sample, all oysters were measured and classified into 5-mm size bins. Beginning in 2003, for each sample, individual oyster lengths were recorded to the nearest $\mathrm{mm}$. Since 1998, samples with $>20 \mathrm{~L}$ of shell have been halved to facilitate processing. The resulting counts and length frequency distributions for each subsample were doubled to estimate density and size distribution on a per $\mathrm{m}^{-2}$ basis when subsampling was necessary. The procedures of Bros and Cowell (1987) were used to assure adequacy of sampling within each strata (reef). All articulated valves of dead oysters, commonly termed boxes (and termed boxes throughout the remainder of this document), were similarly counted and measured.

Mean oyster density (number $\mathrm{m}^{-2}$ ) was calculated for each oyster reef by averaging the number of oysters collected from all samples on a reef within a year and is on a reef-specific basis for the years 1993-2006. Shell volume $\left(\mathrm{L} \mathrm{m}^{-2}\right)$ is reported for the period 1998 to 2006 coincident with the measurement of all boxes. For the period 2002 to 2006, shell was additionally categorized as brown shell, shell that lies above the sediment water interface, and black shell that was exhumed during the collection process.

\section{Biomass Estimation}

Data from a size range (30-139 mm shell length) of live oysters $(n=73)$ collected from Swash (reef 13, Fig. 1) in November $2004(n=24), 2005(n=24)$ and $2006(n=25)$ were used to estimate the relationship between oyster shell length $(\mathrm{mm})$ and biomass or dry tissue weight $(\mathrm{g})$. After the oysters were measured to the nearest $\mathrm{mm}$, the tissue was removed and dried to constant weight (DW, g) at $80^{\circ} \mathrm{C}(72 \mathrm{~h})$. Wet shell weights (WSW, g) were collected from the same 73 oysters used in biomass determinations (see above) after the tissue had been removed and before the shells had air-dried. The relationship between SL and WSW was described.

Biomass calculations were made for each $5 \mathrm{~mm}$ size class for each reef using the mid point of each reef specific, $5 \mathrm{~mm}$ size class as SL in the fitted SL-DW equation and are reported for the period 1998 to 2006, coincident with the period of measurement of all oysters.

\section{Age Structure and Mortality}

Demographic plots were prepared for each year (1998 to 2006) for each reef for both live oysters and boxes using 5-mm bins. Distinct year classes of live oysters that could be followed for a minimum of three years were rare in these plots. The 
period of recruitment to the benthos (also commonly termed spatfall) in the James River results in a broad shell length range within each year class such that interannual junctions are not distinct with a 5-mm size bin. Data for 2003 to 2006 were, as mentioned earlier, available in 1-mm size bins. All data for these years for reefs with densities exceeding 100 oysters $\mathrm{m}^{-2}$ were aggregated on a single size frequency plot with 2-mm bins. The individual cohorts (not year classes, there being one or more cohorts in a single year class) were identified by the method of Bhattacharya (1967). The range and modal length of each cohort was identified by counting cohorts and relating the cohort settlement dates to long term recruitment patterns developed from annual spatfall reports for the James River over the study period (Southworth et al. 1999, Southworth et al. 2000 b, Southworth et al. 2001, Southworth et al. 2002, Southworth et al. 2003, Southworth et al. 2004, Southworth et al. 2005, Southworth et al. 2006, Southworth et al. 2007, available at: http://www.vims.edu/mollusc/publications/mepubamr.htm). The cohorts were thus assigned to years and a linear age-at-length relationship fit to the data $(y=m x+b)$. A linear model is appropriate to describe the early years in an oyster's growth trajectory given the life expectancy of an oyster (10-20 y in undisturbed populations, Powell \& Cummings 1985), their plastic morphology, and lack of adherence to isodiametric form. The standard adoption of an age-at-length plot using the von Bertalanffy (1938) model is not considered appropriate for the current data set.

Using the linear age-at-length relationship, the demographic plots were recast as graphs of year classes for each year and reef for the period 1998 to 2006. Where live cohorts could be followed for more than two successive years, the number of individuals per $\mathrm{m}^{2}$ was recorded in successive year classes. Survivorship and mortality were thus estimated by the following relationships as a proportion with values ranging from $0-1.0$ :

$$
\begin{gathered}
\text { Survivorship }=\# \operatorname{Live}_{(\mathrm{t}+1)} / \# \operatorname{Live}_{(\mathrm{t})} \\
\text { Mortality }=\# \operatorname{Live}_{(\mathrm{t})}-\# \operatorname{Live}_{(\mathrm{t}+1)} / \# \operatorname{Live}_{(\mathrm{t})}
\end{gathered}
$$

Where: \#Live $(t)$ equals the number of live oysters at time $t(t$, units of $1 \mathrm{y}$ ). A possible error inherent to this approach is assignment of oysters to the wrong year class (too old or too young) from the age-at-length relationship. The error cascades through the demographic, and in some instances \#Live $(\mathrm{t}+1)$ \#Live $_{(\mathrm{t})}$, resulting in nonsense negative mortality values in the simple proportion estimator. However, the incorrect assignment to year class may not be the only source of error in the proportion estimator. Under-counting of small length, and thus age, classes can give similar errors. The possibility exists that both spat and spat boxes are underestimated by this sampling method, the latter being physically separated during collection. One of the challenges in the James River, where recruitment can occur as late as September (Southworth et al. 2002, Southworth \& Mann 2004, Southworth et al. 2006), is to count spat in October-November patent tong surveys.

Box-length demographics were converted to box-age demographics employing the assumption that all boxes represent mortality in the preceding year to categorize the boxes into the same age classes as the live oysters. Thus, live oysters with lengths $\mathrm{x}$ through $\mathrm{y}$ and boxes with lengths $\mathrm{x}$ through $\mathrm{y}$ are assumed to represent the same year class and are only counted once. If December is the end of the growing season and the surveys are in the preceding October-November, then all boxes represent mortality in that calendar year with the bulk of mortality being in the warmer months (predation, especially on the smaller individuals) and in the late summer (disease). This assumption is central to the approach that all boxes can be used in mortality estimation. The longevity of undisturbed hinges in articulated valves has not been critically examined, and there is no requirement to estimate the "age" of new boxes as proposed by Volstad et al. (2008). We appreciate the possibility that employing spat box densities may underestimate mortality in that length range because articulated spat boxes are fragile (they disarticulate quickly) and predation related mortality does not leave an intact box in very small oysters.

If boxes are assigned to year classes and only counted once then mortality can be estimated by a second relationship as follows, again expressed as a proportion with values ranging from $0-1.0$ :

$$
\text { Mortality }=\#_{B^{\prime}}(\mathrm{t}) /\left[\operatorname{Box}_{(\mathrm{t})}+\text { Hive }_{(\mathrm{t})}\right]
$$

The two mortality estimators are related by use of the \#Live ( $t$ ) value. A comparison of data from both estimators is presented.

\section{Disease Status}

The prevalence and intensity of Perkinsus marinus (Dermo) and Haplosporidium nelsoni (MSX) from 1993 to 2005 at selected locations in the study area is reported by Carnegie \& Burreson (2006). Data for 2006 were obtained from Carnegie (VIMS, EEAH, personal communication, unpublished data). Disease data are presented herein as weighted prevalence (WP) for comparison with mortality data. Weighted prevalence is calculated based on the following formula (Burreson et al. 1988):

$$
\mathrm{WP}=[(0.5 \times \mathrm{R})+(1 \times \mathrm{L})+(3 \times \mathrm{M})+(5 \times \mathrm{H})] / \mathrm{n}
$$

Where $\mathrm{R}, \mathrm{L}, \mathrm{M}$, and $\mathrm{H}$ are the number of oysters with rare, light, medium and heavy infection intensity respectively and $n$ is the total number of oysters tested.

\section{Data Analyses}

Significance levels for all tests were established at alpha $=$ 0.05 a priori. When appropriate, Fisher's parametric posthoc multiple comparison test was used.

The relationship between the presence of live oysters $\left(\mathrm{O}_{\text {live }}\right.$, tongs with live oyster density $\mathrm{m}^{-2}>0$ ) and the presence of oyster shell $\left(\mathrm{S}_{\mathrm{v}}\right.$, tongs with total shell volume $\left.\mathrm{m}^{-2}>0\right)$ was described using a linear relationship:

$$
\begin{aligned}
\mathrm{O}_{\text {live }} & =-7.4975+0.9764^{*} \mathrm{~S}_{\mathrm{v}}, \mathrm{R}^{2}=0.995, \\
n & =2757 \text { data pairs value }
\end{aligned}
$$

We consider the negative intercept to describe a prerequisite of shell habitat before any oysters can be present. Since shell volume is a good predictor of oyster density and shell is not evenly distributed within these habitats, all subsequent ANOVA and ANCOVA analyses do not include patent tongs where values of zero were recorded for shell volume (total, brown, black) or density of live oysters.

Trends in total shell volume (1998 to 2006) were evaluated using a two-factor ANOVA (year $\times$ reef). These data did not satisfy assumptions of homogeneity of variance or normality regardless of the transformation (logarithm, natural logarithm, 


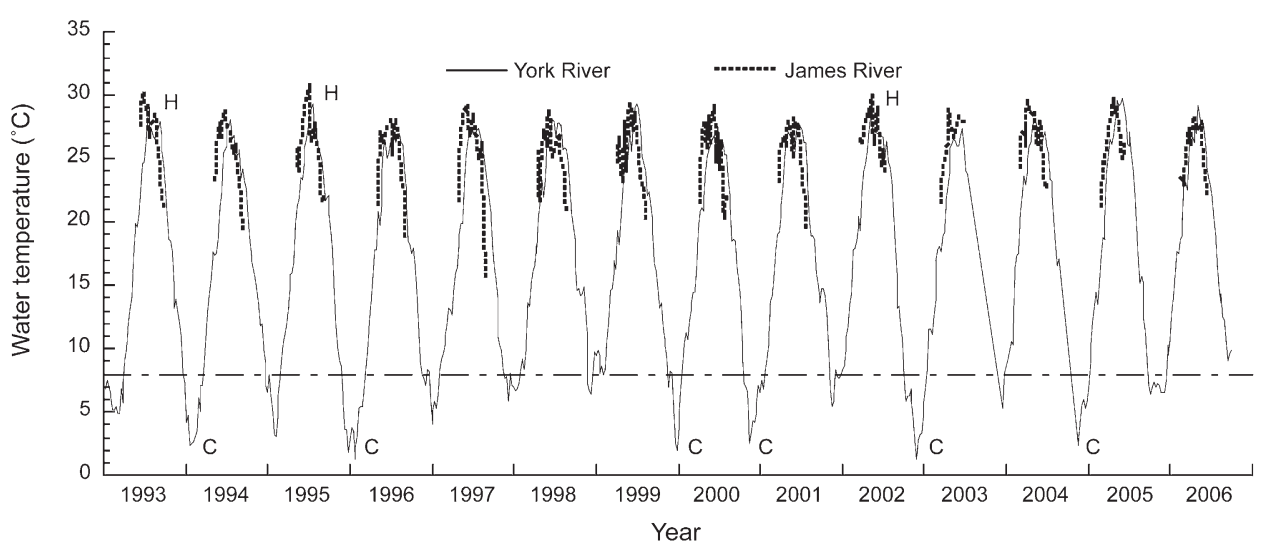

Figure 2. Average weekly bottom temperature in the York River (solid line) and James River (dotted line) 1993 to $2006.8^{\circ} \mathrm{C}$ (dashed horizontal line) is the threshold temperature below which shell growth of $C$. virginica in the James River ceases (Mann \& Evans 2004). C indicates exceptionally cold winters (minima below $3^{\circ} \mathrm{C}$ ) and $\mathrm{H}$ indicates exceptionally warm summers (maxima near $30^{\circ} \mathrm{C}$ ) as discussed in the text.

reciprocal, square root) given the potential range of shell volume values that are typically observed $(0.01$ to $>15 \mathrm{~L})$.

Trends in the proportion of brown shell volume $\left(\mathrm{L} \mathrm{m}^{-2}\right)$ with regard to total shell volume $\left(\mathrm{L} \mathrm{m}^{-2}\right)$ during 2002 to 2006 were evaluated using a two factor ANOVA (reef $\times$ year). These data did not satisfy assumptions of normality or homogeneity of variance regardless of the transformation ((logarithm, natural logarithm, reciprocal, square root) given the observed range of shell volume values $(0.01$ to $>15 \mathrm{~L})$.

Total shell volume ( $\mathrm{L} \mathrm{m}^{-2} ; 1998$ to 2006) was used as a covariate for two 2-factor ANCOVA analyses (reef $\times$ year) that were performed on the density and biomass data. Neither density nor biomass data satisfied assumptions of homogeneity of variance or normality. A 2-factor ANCOVA was also performed using brown shell volume ( $\left.\mathrm{L} \mathrm{m}^{-2} ; 2002-2006\right)$.

\section{RESULTS}

\section{Temperature and Salinity Data}

Annual variation in average weekly bottom water temperature for the York and James Rivers during 1993 through 2006 is illustrated in Figure 2. As previously mentioned, data for the James River were only available for warmer months (May to October). Temperatures for both rivers follow the same sea- sonal trends, although the James appears to warm marginally earlier in the spring and cool earlier in the fall. Nonetheless we suggest that York River data can be used as a surrogate where James River data are unavailable for the purposes of examining interannual differences. Winter minima in the York River vary between 1 and $6^{\circ} \mathrm{C}$ with notable cold winters in 1993 to 1994 , 1995 to 1996, 1999 to 2000, 2000 to 2001, 2002 to 2003 and 2004 to 2005 where minima were below $3{ }^{\circ} \mathrm{C}$ (Fig. 2). By contrast the water temperatures in the winters of 1997 to 1998,1998 to 1999 , 2001 to 2002, 2003 to 2004, and 2005 to 2006 remained at or above $6^{\circ} \mathrm{C}$. Summer maxima vary between $30^{\circ} \mathrm{C}$ for the highest values in 1993, 1995 and 2002, and lower values of approximately $28^{\circ} \mathrm{C}$ in 1996, 2001, and 2006 (Fig. 2).

Annual variation in salinity for the James River from 1993 through 2006, estimated from daily river flow at Richmond using the method of Mann \& Evans (1998), is illustrated in Figure 3. The James River drains a watershed area of approximately 27,000 $\mathrm{km}^{2}$ (Chesapeake Bay Program; http:// www.chesapeakebay.net) with yearly average stream flow ranging from 4,400-21,500 $\mathrm{ft}^{3} / \mathrm{sec}$ (US Geological Survey data; http://nwis.waterdata.usgs.gov). At the upstream station (Fig. 1, reef 1,) annual minima occur in February to March with estimated salinities as low as 2 ppt. Annual maxima at reef 1 typically occur in mid to late summer and vary between $6 \mathrm{ppt}$ (1996) and 13 ppt (1999 and 2002). At the downstream station

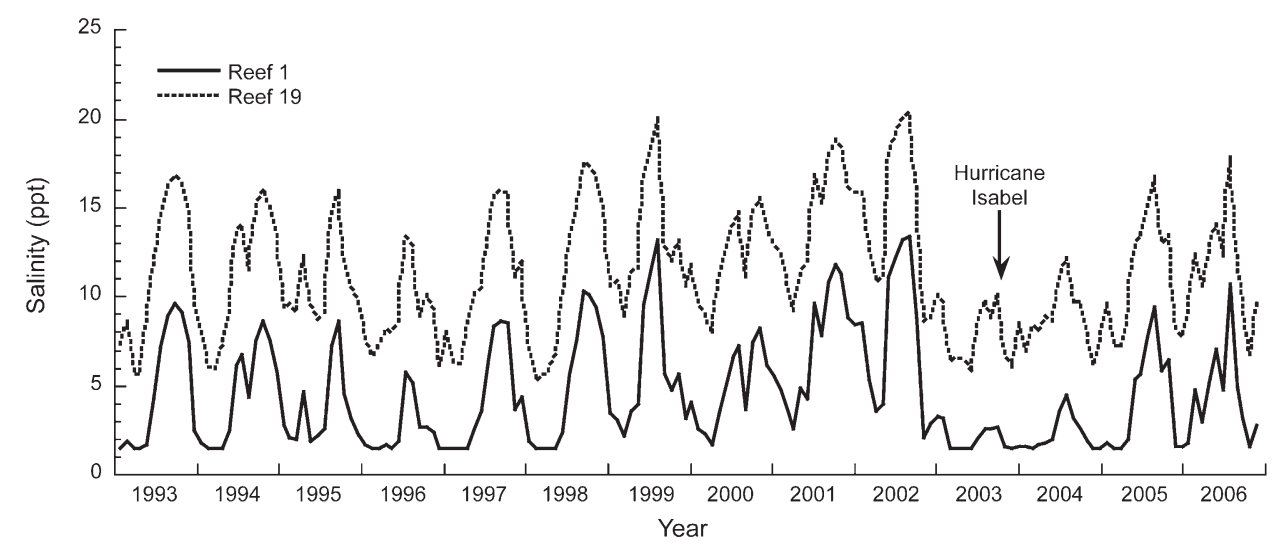

Figure 3. Salinity at reefs 1 (solid line) and 19 (dotted line) estimated from flow data at Richmond (http://waterdata.usgs.gov/nwis/sw) as per Mann \& Evans (1998). Estimate is at a depth of $3 \mathrm{~m}$ for both reefs. 


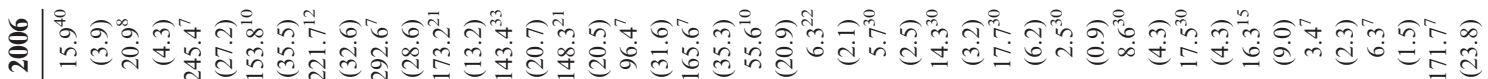

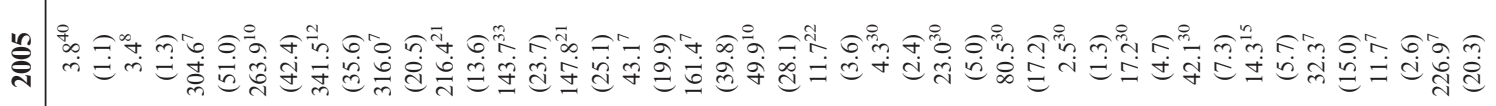

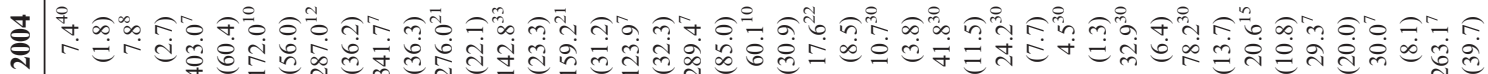

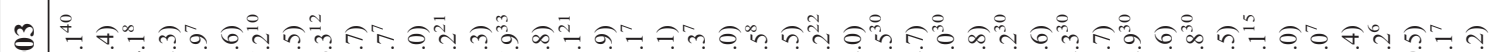

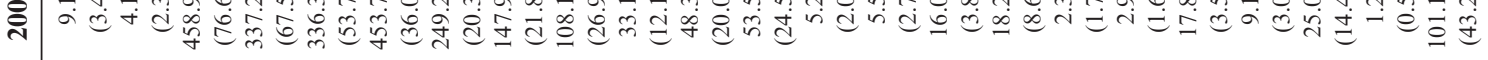

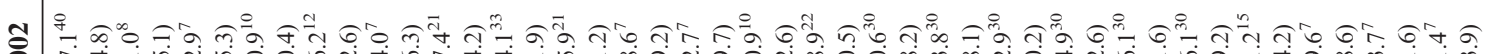

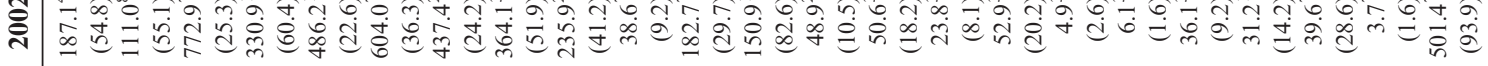

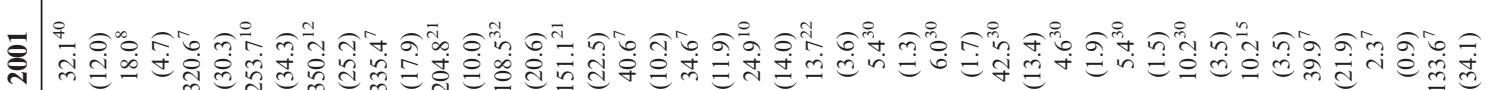

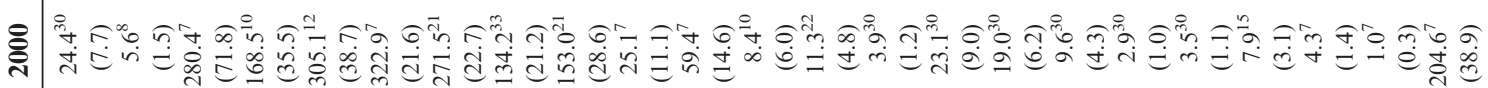

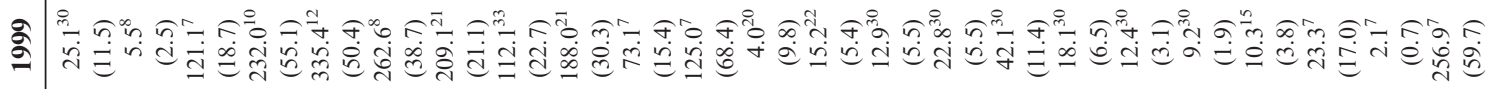

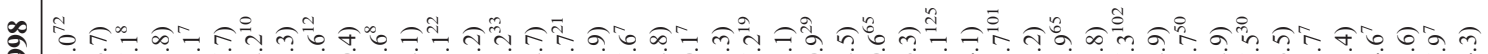

mं

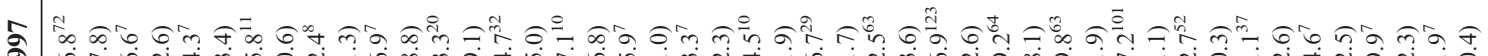
פ

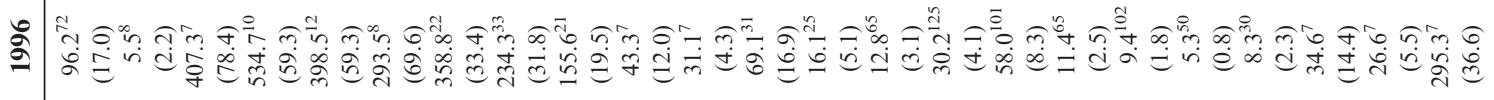

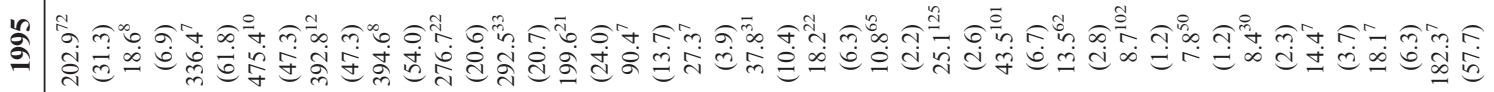

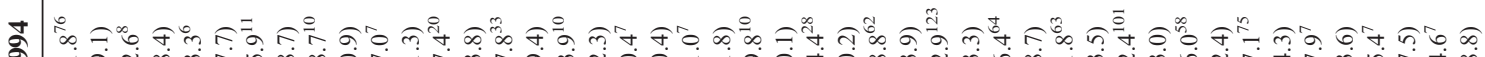
ま में

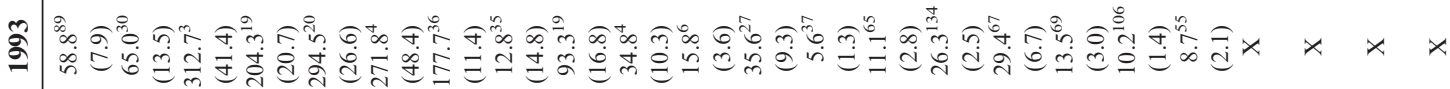

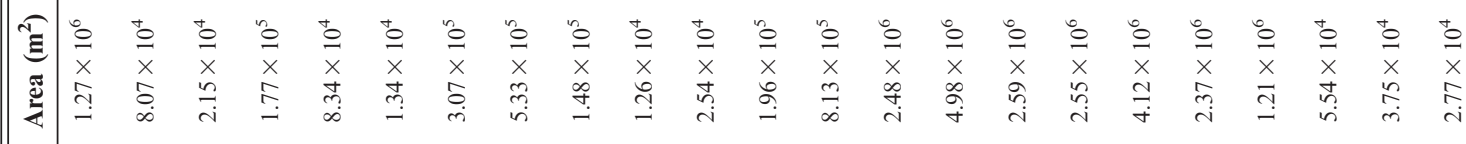

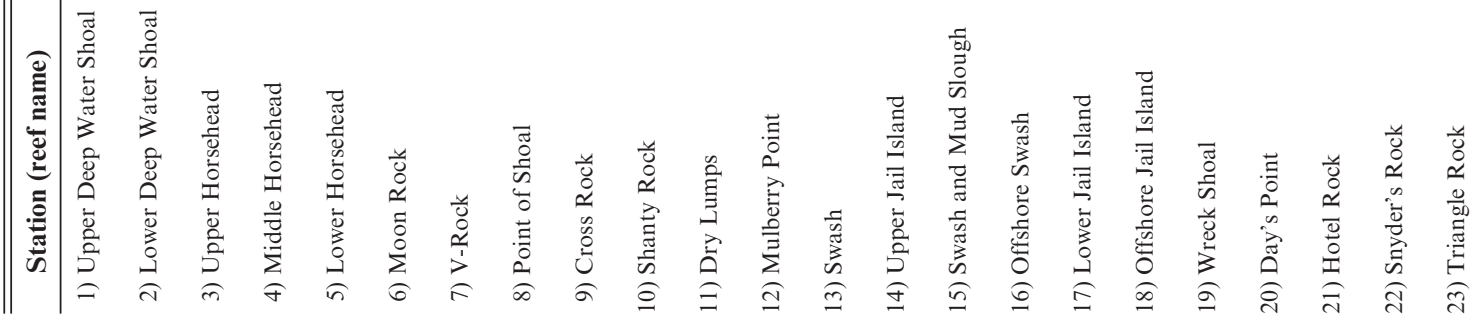


(Fig. 1, reef 19) late winter minima vary between 5 ppt (1998, 2003, and 2005) and 9 ppt (1995, 1999, and 2001) with one extremely low flow year in 2002 when winter minima did not fall below 11 ppt. Summer maxima at reef 19 ranges between 10 (2003) and 20 ppt (1999 and 2002). The 1993 to 1998 period is characterized by these typical seasonal oscillations.

Beginning in summer 1998 a period of higher than average salinities was sustained until spring of 2003. During this time period, salinities at reef 19 , the most downriver site, were always greater than 8-9 ppt. Mid summer to early fall salinities in the 16-20 ppt range were consistently observed in 1998, 1999, 2001, and 2002. By contrast the 2003 to 2004 period was characterized by low salinities that remained below 9 ppt for all but two months in 2002 and three months in 2003. In particular, the 2003 river flow resulted in salinity values that were several ppt lower than average for the majority of the oyster spawning season (June through September, Southworth et al. 2004). The impacts of the lower salinity conditions were exacerbated by Hurricane Isabel's track across the region on September 18, 2003 with concurrent heavy rainfall. The years of 2005 and 2006 marked a return to more typical annual seasonal oscillations.

\section{Description of the Oyster Resource: Density and Biomass}

Changes in reef-specific oyster density for the period 1993 to 2006 are summarized in Table 1. The area of the individual reefs (sampling strata) varied by almost two orders of magnitude. In Burwell's Bay (Fig. 1), the river channel is bordered by a series of moderate sized reefs (reef numbers 1-11, and 20-23), and a suite of reefs within a continuous swath on the northeastern side of Burwell Bay (reef numbers 12-19 inclusive). The former, range in individual size from $1.26 \times 10^{4} \mathrm{~m}^{2}$ to $5.33 \times 10^{5} \mathrm{~m}^{2}$. The latter are larger, ranging from $8.15 \times 10^{5}$ to $4.98 \times 10^{6} \mathrm{~m}^{2}$.

Reefs can be generally described in groups, based on density of live oysters (Table 5, ANCOVA see later). Reefs 3 through 6 exhibit, with very few exceptions, mean densities well in excess of 200 oysters $\mathrm{m}^{-2}$, typically between 300 and 500 oysters $\mathrm{m}^{-2}$, and reaching maximum values of 773 oysters $\mathrm{m}^{-2}$ at reef 3 in 2002 (Table 1). Reefs 4, 7 and 23 are characterized by mean densities in the range of 100-350 oysters $\mathrm{m}^{-2}$ with notable peaks at reef 4 in $1996\left(535\right.$ oysters $\left.\mathrm{m}^{-2}\right)$, reef 7 in $2002\left(437\right.$ oysters $\mathrm{m}^{-2}$ ) and reef 23 in $2002\left(501\right.$ oysters $\mathrm{m}^{-2}$ ). Densities at reefs 8 and 9 are typically in the 100-200 oysters $\mathrm{m}^{-2}$ range with higher values observed in 1994 and 2002 at reef 8 (293 and 364 oysters $\mathrm{m}^{-2}$, respectively), and 1998 and 2002 at reef 9 (210 and 236 oysters $\mathrm{m}^{-2}$, respectively). With only six exceptions (reef 1 in 1995 and 2002, reef 2 in 2002, and reef 10 in 1994, 1998, and 2004), the remaining reef and year combinations all exhibit mean live oyster densities of $<100 \mathrm{~m}^{-2}$, with many values $<20 \mathrm{~m}^{-2}$.

The observed trends in density reflect both recruitment and mortality events. Gradually increasing mean densities were observed in the 1993 to 1996 period, assisted by good recruitment in 1995 and 1996. A trend of generally decreasing density followed through 1998 with variable densities being observed until 2001. A large recruitment event was observed throughout the river in 2002 (Table 5 see later, ANCOVA year $2002>$ all others, see also Southworth et al. 2003), producing the highest densities recorded during the study period at many reefs examined. A decrease in density occurred in 2003 through 2006, with 2006 reef specific values often being the lowest observed in the study period.
Uniformity or patchiness in oyster distribution within the targeted reefs can be examined through variance to mean ratios (Fig. 4). Variance to mean ratios $>1$ indicate aggregation or patchiness, values equal to 1 describe a random distribution, and values $<1$ describe a uniformly distributed resource. The observed variance to mean ratios for live oyster densities from the James River are typically between 10 and 100 (Fig. 4A,) indicating a patchy distribution of the living oyster resource throughout the sites examined. A limited number of reef/year combinations display even higher variance to mean ratios, for all reefs and years. This pattern appears to be independent of oyster density, total shell volume, and brown shell volume (Fig. 4A, 4B and 4C respectively, Table 5 see later). The observed variance to mean ratios of $10-100$ for live oyster density estimates in the James River are comparable to those reported for hard clam (Mercenaria mercenaria, Fegley 2001) and aplacophoran (Scheltema 1985) populations.

The relationship between oyster shell length $(\mathrm{mm})$ and oyster biomass (dry tissue weight g) for 73 oysters ranging from 30-139 $\mathrm{mm}$ shell length was described using a power equation:
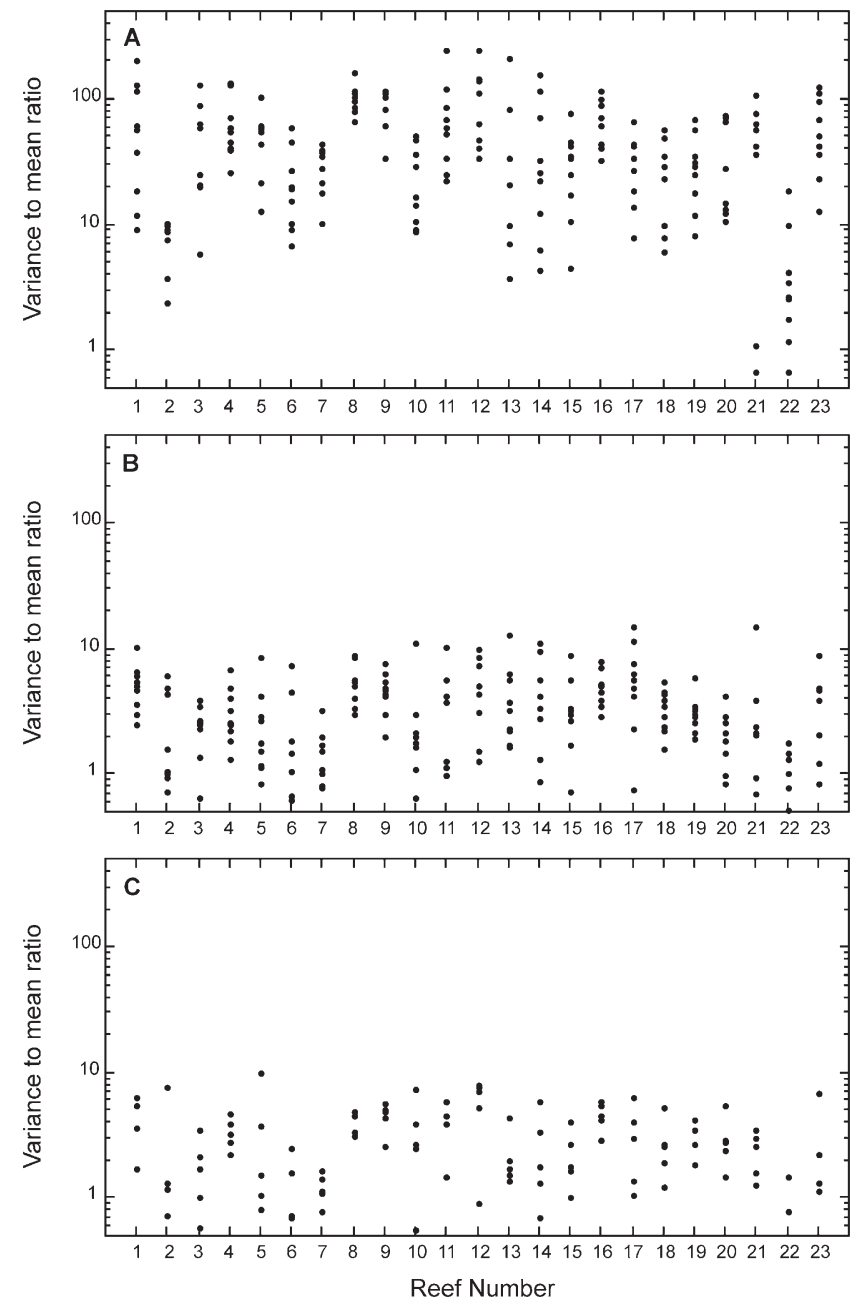

Figure 4. Variance to mean ratios of oyster density (A; 1998 to 2006), total shell volume (B; 1998 to 2006) and brown shell volume (C; 2002 to 2006) for the 23 reefs in the James River. 
TABLE 2.

Average oyster biomass (g dry tissue weight) in the James River from 1998 to 2006. Data are presented as average biomass of oysters $\mathrm{m}^{-2}$ (standard error of the mean appear in parenthesis and $n$ values appear as a superscript for each mean value).

The numbers for the individual reefs correspond to those in Figure 1.

\begin{tabular}{|c|c|c|c|c|c|c|c|c|c|c|}
\hline Station (reef name) & Area $\left(m^{2}\right)$ & 1998 & 1999 & 2000 & 2001 & 2002 & 2003 & 2004 & 2005 & 2006 \\
\hline \multirow[t]{2}{*}{ 1) Upper Deep Water Shoal } & $1.27 \times 10^{6}$ & $14.0^{72}$ & $10.8^{30}$ & $14.8^{30}$ & $12.3^{40}$ & $18.1^{40}$ & $1.4^{40}$ & $1.6^{40}$ & $0.9^{40}$ & $4.1^{40}$ \\
\hline & & $(3.1)$ & $(5.2)$ & $(5.1)$ & $(4.9)$ & $(5.8)$ & $(0.5)$ & $(0.4)$ & $(0.4)$ & $(1.1)$ \\
\hline \multirow[t]{2}{*}{ 2) Lower Deep Water Shoal } & $8.07 \times 10^{4}$ & $2.5^{8}$ & $1.7^{8}$ & $2.8^{8}$ & $2.3^{8}$ & $8.3^{8}$ & $0.8^{8}$ & $1.9^{8}$ & $1.2^{8}$ & $7.1^{8}$ \\
\hline & & $(1.0)$ & $(0.8)$ & $(1.0)$ & $(0.8)$ & $(4.4)$ & $(0.5)$ & $(0.6)$ & $(0.5)$ & (1.8) \\
\hline \multirow[t]{2}{*}{ 3) Upper Horsehead } & $2.15 \times 10^{4}$ & $103.9^{7}$ & $49.5^{7}$ & $97.9^{7}$ & $159.3^{7}$ & $73.3^{7}$ & $79.1^{7}$ & $124.8^{7}$ & $195.1^{7}$ & $134.0^{7}$ \\
\hline & & $(8.7)$ & $(11.3)$ & $(27.5)$ & $(9.8)$ & $(6.9)$ & $(16.6)$ & $(26.3)$ & $(35.6)$ & $(14.9)$ \\
\hline \multirow[t]{2}{*}{ 4) Middle Horsehead } & $1.77 \times 10^{5}$ & $123.7^{10}$ & $67.9^{10}$ & $54.0^{10}$ & $98.4^{10}$ & $43.3^{10}$ & $58.6^{10}$ & $59.1^{10}$ & $152.1^{10}$ & $92.1^{10}$ \\
\hline & & $(12.6)$ & $(15.8)$ & $(11.9)$ & $(19.9)$ & $(9.0)$ & (11.6) & $(19.1)$ & $(27.7)$ & $(23.8)$ \\
\hline \multirow[t]{2}{*}{ 5) Lower Horsehead } & $8.34 \times 10^{4}$ & $122.9^{12}$ & $82.6^{12}$ & $92.6^{12}$ & $125.0^{12}$ & $72.3^{12}$ & $58.7^{12}$ & $87.7^{12}$ & $175.3^{12}$ & $138.6^{12}$ \\
\hline & & $(16.1)$ & (10.4) & $(11.0)$ & $(7.6)$ & (3.9) & $(8.4)$ & $(10.9)$ & $(16.5)$ & $(20.5)$ \\
\hline \multirow[t]{2}{*}{ 6) Moon Rock } & $1.34 \times 10^{4}$ & $117.1^{8}$ & $83.8^{8}$ & $121.4^{7}$ & $128.2^{7}$ & $66.9^{7}$ & $74.3^{7}$ & $123.3^{7}$ & $172.9^{7}$ & $181.1^{7}$ \\
\hline & & $(22.8)$ & (13.9) & (4.9) & (8.6) & $(5.0)$ & $(6.7)$ & $(18.0)$ & (11.9) & (19.6) \\
\hline \multirow[t]{2}{*}{ 7) V-Rock } & $3.07 \times 10^{5}$ & $92.4^{22}$ & $51.3^{21}$ & $78.2^{21}$ & $76.5^{21}$ & $46.0^{21}$ & $50.7^{21}$ & $94.0^{21}$ & $115.3^{21}$ & $86.7^{21}$ \\
\hline & & $(6.5)$ & $(5.0)$ & $(5.8)$ & $(5.3)$ & $(2.8)$ & $(3.7)$ & (7.6) & (8.4) & (8.6) \\
\hline \multirow[t]{2}{*}{ 8) Point of Shoal } & $5.33 \times 10^{5}$ & $60.5^{33}$ & $37.8^{33}$ & $47.9^{33}$ & $47.1^{32}$ & $29.6^{33}$ & $32.2^{33}$ & $57.7^{33}$ & $78.5^{33}$ & $79.6^{33}$ \\
\hline & & (8.6) & (7.9) & $(7.7)$ & $(8.8)$ & $(4.3)$ & $(4.7)$ & $(9.2)$ & $(12.0)$ & (11.1) \\
\hline \multirow[t]{2}{*}{ 9) Cross Rock } & $1.48 \times 10^{5}$ & $38.7^{21}$ & $34.8^{21}$ & $37.8^{21}$ & $46.2^{21}$ & $24.0^{21}$ & $20.8^{21}$ & $34.9^{21}$ & $55.2^{21}$ & $54.0^{21}$ \\
\hline & & $(6.1)$ & $(5.8)$ & $(6.6)$ & $(7.1)$ & $(4.0)$ & $(4.8)$ & $\left(8.3^{6}\right)$ & (10.4) & $(7.8)$ \\
\hline \multirow{2}{*}{ 10) Shanty Rock } & $1.26 \times 10^{4}$ & $18.5^{7}$ & $10.7^{7}$ & $5.9^{7}$ & $6.3^{7}$ & $4.3^{7}$ & $8.6^{7}$ & $11.0^{7}$ & $11.1^{7}$ & $31.8^{7}$ \\
\hline & & $(5.4)$ & $(2.2)$ & (2.6) & (1.6) & (1.4) & $(3.1)$ & (3.3) & $(4.7)$ & (10.6) \\
\hline \multirow{2}{*}{ 11) Dry Lumps } & $2.54 \times 10^{4}$ & $6.0^{7}$ & $14.3^{7}$ & $11.9^{7}$ & $4.6^{7}$ & $25.5^{7}$ & $11.1^{7}$ & $36.9^{7}$ & $36.8^{7}$ & $40.1^{7}$ \\
\hline & & $(2.8)$ & $(8.4)$ & $(3.3)$ & (1.8) & $(4.5)$ & $(4.3)$ & (14.6) & $(9.1)$ & $(8.0)$ \\
\hline \multirow[t]{2}{*}{ 12) Mulberry Point } & $1.96 \times 10^{5}$ & $21.2^{19}$ & $8.4^{20}$ & $4.0^{10}$ & $10.7^{10}$ & $11.8^{10}$ & $8.3^{8}$ & $17.9^{10}$ & $27.2^{10}$ & $38.1^{10}$ \\
\hline & & $(7.3)$ & $(5.1)$ & $(2.7)$ & $(6.2)$ & $(6.6)$ & $(3.9)$ & $(8.9)$ & (16.4) & $(15.0)$ \\
\hline \multirow[t]{2}{*}{ 13) Swash } & $8.13 \times 10^{5}$ & $9.4^{29}$ & $6.7^{22}$ & $4.5^{22}$ & $3.4^{22}$ & $3.6^{22}$ & $0.8^{22}$ & $5.3^{22}$ & $6.4^{22}$ & $3.6^{22}$ \\
\hline & & $(5.5)$ & $(2.4)$ & $(1.8)$ & $(1.0)$ & $(1.0)$ & $(0.3)$ & $(2.6)$ & $(2.1)$ & $(1.2)$ \\
\hline \multirow[t]{2}{*}{ 14) Upper Jail Island } & $2.48 \times 10^{6}$ & $10.5^{65}$ & $4.8^{30}$ & $1.4^{30}$ & $1.3^{30}$ & $3.0^{30}$ & $1.0^{30}$ & $2.9^{30}$ & $2.3^{30}$ & $2.4^{30}$ \\
\hline & & $(3.5)$ & $(2.2)$ & $(0.5)$ & $(0.3)$ & $(0.9)$ & $(0.5)$ & $(1.0)$ & $(1.3)$ & $(1.0)$ \\
\hline \multirow[t]{2}{*}{ 15) Swash and Mud Slough } & $4.98 \times 10^{6}$ & $14.3^{125}$ & $7.4^{30}$ & $6.8^{30}$ & $1.5^{30}$ & $2.5^{30}$ & $3.7^{30}$ & $10.5^{30}$ & $10.5^{30}$ & $5.9^{30}$ \\
\hline & & $(1.3)$ & $(1.9)$ & $(2.7)$ & $(0.5)$ & $(0.9)$ & $(0.9)$ & $(3.0)$ & $(2.1)$ & $(1.5)$ \\
\hline \multirow[t]{2}{*}{ 16) Offshore Swash } & $2.59 \times 10^{6}$ & $11.2^{101}$ & $7.2^{30}$ & $5.2^{30}$ & $10.9^{30}$ & $4.6^{30}$ & $3.4^{30}$ & $3.9^{30}$ & $23.1^{30}$ & $6.9^{30}$ \\
\hline & & $(2.0)$ & $(1.8)$ & $(1.7)$ & (3.6) & $(1.9)$ & $(1.7)$ & $(1.5)$ & $(6.1)$ & $(2.7)$ \\
\hline \multirow[t]{2}{*}{ 17) Lower Jail Island } & $2.55 \times 10^{6}$ & $5.0^{65}$ & $3.4^{30}$ & $3.1^{30}$ & $1.1^{30}$ & $0.6^{30}$ & $0.7^{30}$ & $0.5^{30}$ & $1.7^{30}$ & $0.7^{30}$ \\
\hline & & $(1.0)$ & $(1.2)$ & $(1.2)$ & $(0.5)$ & $(0.3)$ & $(0.5)$ & $(0.2)$ & $(0.9)$ & $(0.3)$ \\
\hline \multirow[t]{2}{*}{ 18) Offshore Jail Island } & $4.12 \times 10^{6}$ & $2.8^{102}$ & $2.3^{30}$ & $0.9^{30}$ & $1.3^{30}$ & $1.0^{30}$ & $0.9^{30}$ & $2.2^{30}$ & $5.1^{30}$ & $2.7^{30}$ \\
\hline & & $(0.4)$ & $(0.5)$ & $(0.3)$ & $(0.4)$ & $(0.3)$ & $(0.4)$ & $(0.5)$ & $(1.4)$ & (1.4) \\
\hline \multirow[t]{2}{*}{ 19) Wreck Shoal } & $2.37 \times 10^{6}$ & $1.4^{50}$ & $1.5^{30}$ & $0.9^{30}$ & $1.8^{30}$ & $2.5^{30}$ & $4.1^{30}$ & $9.5^{30}$ & $12.4^{30}$ & $6.1^{30}$ \\
\hline & & $(0.2)$ & $(0.4)$ & $(0.3)$ & $(0.7)$ & $(0.7)$ & $(0.9)$ & $(2.4)$ & $(2.3)$ & $(1.5)$ \\
\hline \multirow[t]{2}{*}{ 20) Day’s Point } & $1.21 \times 10^{6}$ & $2.4^{30}$ & $2.3^{15}$ & $1.9^{15}$ & $1.5^{15}$ & $2.4^{15}$ & $2.5^{15}$ & $4.8^{15}$ & $5.4^{15}$ & $7.6^{15}$ \\
\hline & & $(0.6)$ & $(0.9)$ & $(0.7)$ & $(0.4)$ & $(1.2)$ & $(0.8)$ & $(3.0)$ & $(2.4)$ & $(4.5)$ \\
\hline 21) Hotel Rock & $5.54 \times 10^{4}$ & $6.8^{7}$ & $5.2^{7}$ & $1.1^{7}$ & $3.1^{7}$ & $2.9^{7}$ & $5.4^{7}$ & $6.4^{7}$ & $17.4^{7}$ & $1.7^{7}$ \\
\hline & & $(3.0)$ & (3.3) & $(0.4)$ & $(1.9)$ & $(2.0)$ & $(3.1)$ & $(3.9)$ & $(9.0)$ & $(1.1)$ \\
\hline 22) Snyder's Rock & $3.75 \times 10^{4}$ & $6.9^{7}$ & $0.9^{7}$ & $0.4^{7}$ & $0.3^{7}$ & $0.7^{7}$ & $0.2^{6}$ & $1.0^{7}$ & $2.6^{7}$ & $3.3^{7}$ \\
\hline & & $(2.8)$ & $(0.3)$ & $(0.2)$ & $(0.1)$ & $(0.3)$ & $(0.1)$ & $(0.3)$ & $(0.5)$ & $(0.9)$ \\
\hline 23) Triangle Rock & $2.77 \times 10^{4}$ & $80.5^{7}$ & $79.3^{7}$ & $79.9^{7}$ & $54.8^{7}$ & $54.0^{7}$ & $20.0^{7}$ & $109.0^{7}$ & $159.3^{7}$ & $137.7^{7}$ \\
\hline & & $(23.5)$ & $(16.8)$ & $(14.8)$ & $(13.6)$ & $(8.4)$ & $(8.1)$ & $(16.1)$ & $(15.8)$ & $(26.8)$ \\
\hline
\end{tabular}

$$
\begin{aligned}
\operatorname{Biomass}(\mathrm{g}) & =0.0000712^{*} \text { Shell Length }(\mathrm{mm})^{2.15} ; \\
\mathrm{R}^{2} & =0.80, n=73
\end{aligned}
$$

Year and reef specific demographic data were used to generate a description of reef specific oyster biomass ( $g$ dry tissue) for the period 1998 to 2006 as summarized in Table 2. The grouping of reefs previously described for density (Table 5 see later, described above) also applies for biomass and brown shell volume (Table 5 see later, ANCOVA, Fisher's test). Note the similarity in grouping of the reefs with respect to both density and biomass.

Reefs 3 through 6 and reef 23 exhibit, with only two exceptions, mean biomass values in excess of $50 \mathrm{~g} \mathrm{~m}^{-2}$ and at least one value in excess of $100 \mathrm{~g} \mathrm{~m}^{-2}$ (Table 2). All reefs in this group were in the range $155-195 \mathrm{~g} \mathrm{~m}^{-2}$ in 2005 , with the $195 \mathrm{~g} \mathrm{~m}^{-2}$ value at reef 3 being the highest recorded in the study period. Note that the highest recruitment year (2002, see Table 1) occurred during the lowest biomass year when the latter varied between $43.3 \mathrm{~g} \mathrm{~m}^{-2}$ (the lowest value for the study period for this group) and $73.3 \mathrm{~g} \mathrm{~m}^{-2}$. High biomass records in this group during 2003 through 2006 reflect oyster survival and growth from the 2002 recruitment event. Reefs 7 and 8 are characterized by mean biomass values between 29.6 and $115.3 \mathrm{~g} \mathrm{~m}^{-2}$. Notably the lowest value occurred at reef 8 in 2002 despite a very high density $\left(364 \mathrm{~m}^{-2}\right)$. This recruitment event in the presence of few adults (Table 1) resulted in the highest biomass records for these 
reefs in the 2004 through 2006 period. With only nine exceptions (reef 10 in 2006, reef 11 in 2002 and 2004-2006, reef 12 in 1998 and 2005 to 2006, and reef 16 in 2005), the remaining reef and year combinations all exhibit mean biomass values of $<20 \mathrm{~g} \mathrm{~m}^{-2}$

\section{Description of the Shell Resource: Density}

Live oysters and dead shell material provide settlement substrate for new recruits and complex habitat for reef associated organisms. Shell material observed in the surveys and quantified on a per $\mathrm{L}$ basis for each sample $\left(\mathrm{m}^{2}\right)$ range in size from intact oyster shells to shell fragments ("hash").

The recorded reef and year specific values of total shell volume (L, brown combined with black) and brown shell volume for the study period are given in Table 3 (1998 to 2006) and Table 4 (2002 to 2006) respectively in units of $\mathrm{L} \mathrm{m}^{-2}$. Brown shell volume is an integrated estimator of recent mortality over a limited number of years. The time frame for decay or disappearance of brown shell may be on the order of years (Powell et al. 2006). Black shell results from burial of brown shell at some juncture in this process, and the decay or disappearance rate may then change. A gradation in total shell volume with reef was evident (Table 5, ANOVA). Reefs 5 and 6 were consistently in excess of $21 \mathrm{~L} \mathrm{~m}^{-2}$, in both cases recording one value in excess of $30 \mathrm{~L} \mathrm{~m}^{-2}$. This was significantly higher than reefs $3,4,7,8$, and 23 (Table 5) that were typically between 17 and $23 \mathrm{~L} \mathrm{~m}^{-2}$ with a limited number of values as low as $10.1 \mathrm{~L} \mathrm{~m}^{-2}$ (reef 23 in 2003) and 12.6 $\mathrm{L} \mathrm{m}^{-2}$ (reef 3 in 1999). Reefs 9, 10, and 11 were consistently

TABLE 3.

Average total volume (liters) of oyster shell in the James River from 1998 to 2006. Presented as shell volume $\mathbf{m}^{-2}$ (standard error of the mean appear in parenthesis and $n$ values appear as a superscript for each mean value). The numbers for the individual reefs correspond to those in Figure 1.

\begin{tabular}{|c|c|c|c|c|c|c|c|c|c|c|}
\hline Station (reef name) & Area $\left(\mathrm{m}^{2}\right)$ & 1998 & 1999 & 2000 & 2001 & 2002 & 2003 & 2004 & 2005 & 2006 \\
\hline \multirow[t]{2}{*}{ 1) Upper Deep Water Shoal } & $1.27 \times 10^{6}$ & $7.6^{72}$ & $6.5^{30}$ & $7.6^{30}$ & $6.4^{40}$ & $7.0^{40}$ & $4.1^{40}$ & $6.0^{40}$ & $2.8^{40}$ & $4.4^{40}$ \\
\hline & & $(1.1)$ & $(2.0)$ & $(1.7)$ & $(1.7)$ & $(2.0)$ & $(1.1)$ & $(1.1)$ & $(0.7)$ & $(1.0)$ \\
\hline \multirow[t]{2}{*}{ 2) Lower Deep Water Shoal } & $8.07 \times 10^{4}$ & $7.5^{8}$ & $5.4^{8}$ & $6.5^{8}$ & $4.4^{8}$ & $4.0^{8}$ & $4.6^{8}$ & $5.6^{8}$ & $3.0^{8}$ & $4.4^{8}$ \\
\hline & & $(1.4)$ & $(1.8)$ & $(1.3)$ & $(1.5)$ & $(2.5)$ & $(1.1)$ & $(1.1)$ & $(0.6)$ & $(0.9)$ \\
\hline \multirow[t]{2}{*}{ 3) Upper Horsehead } & $2.15 \times 10^{4}$ & $19.6^{7}$ & $12.6^{7}$ & $19.9^{7}$ & $28.6^{7}$ & $32.1^{7}$ & $26.9^{7}$ & $23.4^{7}$ & $22.4^{7}$ & $19.1^{7}$ \\
\hline & & $(2.8)$ & $(2.2)$ & $(3.2)$ & $(3.0)$ & $(2.5)$ & $(3.1)$ & $(2.9)$ & $(3.5)$ & $(1.3)$ \\
\hline \multirow[t]{2}{*}{ 4) Middle Horsehead } & $1.77 \times 10^{5}$ & $25.5^{10}$ & $20.1^{10}$ & $17.8^{10}$ & $23.0^{10}$ & $26.3^{10}$ & $21.9^{10}$ & $17.7^{10}$ & $21.2^{10}$ & $16.2^{10}$ \\
\hline & & (1.8) & $(3.7)$ & $(2.9)$ & $(3.1)$ & $(3.8)$ & $(3.2)$ & $(3.2)$ & $(3.1)$ & $(3.5)$ \\
\hline \multirow[t]{2}{*}{ 5) Lower Horsehead } & $8.34 \times 10^{4}$ & $25.8^{12}$ & $26.8^{12}$ & $22.4^{12}$ & $26.8^{12}$ & $32.7^{12}$ & $24.1^{12}$ & $21.4^{12}$ & $26.1^{12}$ & $22.2^{12}$ \\
\hline & & $(2.4)$ & $(3.5)$ & $(1.5)$ & $(1.4)$ & $(2.0)$ & $(2.3)$ & $(1.4)$ & $(2.0)$ & $(2.8)$ \\
\hline \multirow[t]{2}{*}{ 6) Moon Rock } & $1.34 \times 10^{4}$ & $22.3^{8}$ & $24.5^{8}$ & $27.6^{7}$ & $30.0^{7}$ & $32.9^{7}$ & $28.9^{7}$ & $22.9^{7}$ & $26.0^{7}$ & $28.0^{7}$ \\
\hline & & $(4.5)$ & $(3.7)$ & $(1.6)$ & $(0.8)$ & $(2.6)$ & $(2.1)$ & (1.4) & $(0.9)$ & $(2.7)$ \\
\hline \multirow[t]{2}{*}{ 7) V-Rock } & $3.07 \times 10^{5}$ & $23.4^{22}$ & $16.9^{21}$ & $25.5^{21}$ & $21.4^{21}$ & $27.7^{21}$ & $22.0^{21}$ & $20.6^{21}$ & $21.6^{21}$ & $18.0^{21}$ \\
\hline & & $(1.5)$ & $(1.6)$ & $(1.4)$ & $(1.0)$ & $(1.4)$ & $(1.1)$ & $(0.9)$ & $(0.9)$ & $(1.2)$ \\
\hline \multirow[t]{2}{*}{ 8) Point of Shoal } & $5.33 \times 10^{5}$ & $17.3^{33}$ & $15.6^{33}$ & $19.2^{33}$ & $16.0^{32}$ & $19.2^{33}$ & $19.7^{33}$ & $13.7^{33}$ & $17.4^{33}$ & $17.0^{33}$ \\
\hline & & $(2.0)$ & $(2.6)$ & $(2.7)$ & $(2.3)$ & $(2.4)$ & $(2.0)$ & $(1.7)$ & $(2.0)$ & (1.9) \\
\hline \multirow[t]{2}{*}{ 9) Cross Rock } & $1.48 \times 10^{5}$ & $15.9^{21}$ & $17.3^{21}$ & $15.5^{21}$ & $16.9^{21}$ & $19.2^{21}$ & $11.8^{21}$ & $11.8^{21}$ & $16.4^{21}$ & $16.3^{21}$ \\
\hline & & $(2.0)$ & $(2.3)$ & $(2.5)$ & $(2.2)$ & $(2.8)$ & $(2.0)$ & $(1.8)$ & $(1.7)$ & (1.9) \\
\hline \multirow{2}{*}{ 10) Shanty Rock } & $1.26 \times 10^{4}$ & $13.4^{7}$ & $14.7^{7}$ & $9.3^{7}$ & $12.4^{7}$ & $17.4^{7}$ & $10.1^{7}$ & $7.1^{7}$ & $8.0^{7}$ & $11.7^{7}$ \\
\hline & & $(3.0)$ & $(2.9)$ & (3.8) & $(2.7)$ & $(6.0)$ & $(3.1)$ & (1.4) & (2.6) & $(3.5)$ \\
\hline \multirow[t]{2}{*}{ 11) Dry Lumps } & $2.54 \times 10^{4}$ & $8.0^{7}$ & $14.7^{7}$ & $15.9^{7}$ & $11.9^{7}$ & $19.9^{7}$ & $10.2^{7}$ & $15.1^{7}$ & $12.2^{7}$ & $18.6^{7}$ \\
\hline & & $(2.1)$ & $(5.2)$ & $(1.7)$ & $(2.3)$ & $(0.7)$ & $(2.5)$ & $(4.3)$ & $(2.5)$ & $(1.7)$ \\
\hline \multirow[t]{2}{*}{ 12) Mulberry Point } & $1.96 \times 10^{5}$ & $7.1^{19}$ & $5.7^{20}$ & $2.2^{10}$ & $5.9^{10}$ & $7.1^{10}$ & $7.5^{8}$ & $6.8^{10}$ & $6.1^{10}$ & $7.8^{10}$ \\
\hline & & (1.6) & $(1.5)$ & $(1.0)$ & $(2.2)$ & $(3.8)$ & $(3.0)$ & $(2.6)$ & $(2.4)$ & $(2.9)$ \\
\hline \multirow[t]{2}{*}{ 13) Swash } & $8.13 \times 10^{5}$ & $4.1^{29}$ & $3.4^{22}$ & $2.8^{22}$ & $2.5^{22}$ & $1.9^{22}$ & $0.8^{22}$ & $2.9^{22}$ & $2.9^{22}$ & $1.3^{22}$ \\
\hline & & $(1.4)$ & $(1.1)$ & $(1.1)$ & $(0.8)$ & $(0.5)$ & $(0.3)$ & $(1.0)$ & $(0.9)$ & $(0.5)$ \\
\hline \multirow[t]{2}{*}{ 14) Upper Jail Island } & $2.48 \times 10^{6}$ & $4.1^{65}$ & $5.3^{30}$ & $1.0^{30}$ & $1.8^{30}$ & $4.0^{30}$ & $1.4^{30}$ & $2.2^{30}$ & $1.3^{30}$ & $1.4^{30}$ \\
\hline & & $(0.9)$ & $(1.1)$ & $(0.3)$ & $(0.4)$ & $(1.4)$ & $(0.7)$ & $(0.7)$ & $(0.5)$ & $(0.5)$ \\
\hline \multirow[t]{2}{*}{ 15) Swash and Mud Slough } & $4.98 \times 10^{6}$ & $7.3^{125}$ & $5.1^{30}$ & $3.3^{30}$ & $1.2^{30}$ & $2.8^{30}$ & $3.9^{30}$ & $5.4^{30}$ & $5.2^{30}$ & $2.4^{30}$ \\
\hline & & $(0.5)$ & $(0.9)$ & $(1.4)$ & $(0.3)$ & $(0.9)$ & $(0.8)$ & $(1.2)$ & $(0.9)$ & $(0.5)$ \\
\hline \multirow[t]{2}{*}{ 16) Offshore Swash } & $2.59 \times 10^{6}$ & $6.2^{101}$ & $5.2^{30}$ & $4.0^{30}$ & $7.3^{30}$ & $3.6^{30}$ & $2.9^{30}$ & $2.5^{30}$ & $8.5^{30}$ & $2.7^{30}$ \\
\hline & & $(0.8)$ & $(1.1)$ & $(1.1)$ & $(2.1)$ & $(1.2)$ & $(1.1)$ & $(0.8)$ & $(1.4)$ & $(0.8)$ \\
\hline \multirow[t]{2}{*}{ 17) Lower Jail Island } & $2.55 \times 10^{6}$ & $5.2^{65}$ & $8.3^{30}$ & $3.6^{30}$ & $2.8^{30}$ & $1.3^{30}$ & $0.7^{30}$ & $1.0^{30}$ & $1.0^{30}$ & $1.1^{30}$ \\
\hline & & $(0.8)$ & $(2.1)$ & $(1.4)$ & $(0.9)$ & $(0.6)$ & $(0.5)$ & $(0.3)$ & $(0.5)$ & $(0.3)$ \\
\hline \multirow[t]{2}{*}{ 18) Offshore Jail Island } & $4.12 \times 10^{6}$ & $5.3^{102}$ & $4.6^{30}$ & $1.6^{30}$ & $3.6^{30}$ & $5.7^{30}$ & $2.7^{30}$ & $3.2^{30}$ & $4.0^{30}$ & $2.1^{30}$ \\
\hline & & $(0.5)$ & $(0.7)$ & $(0.5)$ & $(0.9)$ & $(1.3)$ & $(0.7)$ & $(0.6)$ & $(0.7)$ & $(0.7)$ \\
\hline \multirow[t]{2}{*}{ 19) Wreck Shoal } & $2.37 \times 10^{6}$ & $6.1^{50}$ & $16.0^{30}$ & $4.6^{30}$ & $8.9^{30}$ & $9.0^{30}$ & $9.0^{30}$ & $9.0^{30}$ & $6.7^{30}$ & $7.5^{30}$ \\
\hline & & $(0.6)$ & $(1.9)$ & $(1.0)$ & $(1.1)$ & $(1.3)$ & $(1.1)$ & $(1.1)$ & $(0.9)$ & $(1.2)$ \\
\hline \multirow[t]{2}{*}{ 20) Day’s Point } & $1.21 \times 10^{6}$ & $4.4^{30}$ & $4.7^{15}$ & $4.6^{15}$ & $5.5^{15}$ & $8.6^{15}$ & $6.8^{15}$ & $4.6^{15}$ & $4.1^{15}$ & $5.9^{15}$ \\
\hline & & $(0.8)$ & $(1.3)$ & $(1.3)$ & $(1.3)$ & $(2.3)$ & $(1.6)$ & $(1.2)$ & $(1.6)$ & (1.7) \\
\hline 21) Hotel Rock & $5.54 \times 10^{4}$ & $6.5^{7}$ & $6.5^{7}$ & $4.9^{7}$ & $7.9^{7}$ & $6.7^{7}$ & $5.6^{7}$ & $4.6^{7}$ & $7.0^{7}$ & $2.0^{7}$ \\
\hline & & $(2.7)$ & $(4.1)$ & $(1.4)$ & $(3.2)$ & $(3.6)$ & $(2.3)$ & $(2.2)$ & $(2.0)$ & $(1.3)$ \\
\hline 22) Snyder's Rock & $3.75 \times 10^{4}$ & $9.0^{7}$ & $5.0^{7}$ & $3.3^{7}$ & $3.3^{7}$ & $1.5^{7}$ & $2.6^{6}$ & $2.3^{7}$ & $3.5^{7}$ & $2.0^{7}$ \\
\hline & & $(1.1)$ & $(1.0)$ & $(0.4)$ & $(0.8)$ & $(0.5)$ & $(0.5)$ & $(0.6)$ & $(0.9)$ & $(0.4)$ \\
\hline 23) Triangle Rock & $2.77 \times 10^{4}$ & $19.9^{7}$ & $22.3^{7}$ & $25.4^{7}$ & $18.0^{7}$ & $28.3^{7}$ & $10.1^{7}$ & $26.6^{7}$ & $26.6^{7}$ & $26.1^{7}$ \\
\hline & & $(4.9)$ & $(4.0)$ & $(4.1)$ & $(4.3)$ & $(2.9)$ & $(3.9)$ & $(1.8)$ & $(0.6)$ & $(2.1)$ \\
\hline
\end{tabular}


TABLE 4.

Average volume (liters) of brown oyster shell in the James River from 2002 to 2006 . Presented as shell volume $\mathrm{m}^{-2}$ (standard error of the mean appear in parenthesis and $n$ values appear as a superscript for each mean value). The numbers for the individual reefs correspond to those in Figure 1.

\begin{tabular}{|c|c|c|c|c|c|c|}
\hline Station (Reef Name) & Area $\left(\mathrm{m}^{2}\right)$ & 2002 & 2003 & 2004 & 2005 & 2006 \\
\hline \multirow[t]{2}{*}{ 1) Upper Deep Water Shoal } & $1.27 \times 10^{6}$ & $6.0^{40}$ & $3.2^{40}$ & $4.7^{40}$ & $1.6^{40}$ & $3.0^{40}$ \\
\hline & & $(0.3)$ & $(0.1)$ & $(0.1)$ & $(0.1)$ & $(0.1)$ \\
\hline \multirow[t]{2}{*}{ 2) Lower Deep Water Shoal } & $8.07 \times 10^{4}$ & $3.3^{8}$ & $3.1^{8}$ & $3.6^{8}$ & $1.2^{8}$ & $3.4^{8}$ \\
\hline & & $(0.8)$ & $(0.3)$ & $(0.3)$ & $(0.1)$ & $(0.1)$ \\
\hline \multirow[t]{2}{*}{ 3) Upper Horsehead } & $2.15 \times 10^{4}$ & $30.3^{7}$ & $24.0^{7}$ & $21.1^{7}$ & $19.9^{7}$ & $18.4^{7}$ \\
\hline & & $(0.8)$ & $(0.9)$ & $(1.0)$ & $(1.2)$ & $(0.5)$ \\
\hline \multirow[t]{2}{*}{ 4) Middle Horsehead } & $1.77 \times 10^{5}$ & $20.7^{10}$ & $17.6^{10}$ & $14.7^{10}$ & $17.7^{10}$ & $14.8^{10}$ \\
\hline & & $(1.2)$ & $(0.8)$ & $(0.9)$ & $(0.9)$ & $(1.0)$ \\
\hline \multirow[t]{2}{*}{ 5) Lower Horsehead } & $8.34 \times 10^{4}$ & $29.2^{12}$ & $23.4^{12}$ & $17.6^{12}$ & $20.4^{12}$ & $20.6^{12}$ \\
\hline & & $(0.4)$ & $(1.3)$ & $(0.4)$ & $(0.5)$ & $(0.7)$ \\
\hline \multirow[t]{2}{*}{ 6) Moon Rock } & $1.34 \times 10^{4}$ & $27.1^{7}$ & $22.0^{7}$ & $18.9^{7}$ & $21.1^{7}$ & $25.7^{7}$ \\
\hline & & $(0.6)$ & $(0.8)$ & $(0.5)$ & $(0.4)$ & $(1.1)$ \\
\hline \multirow[t]{2}{*}{ 7) V-Rock } & $3.07 \times 10^{5}$ & $23.3^{21}$ & $18.6^{21}$ & $17.7^{21}$ & $17.1^{21}$ & $17.0^{21}$ \\
\hline & & $(0.3)$ & $(0.2)$ & $(0.2)$ & $(0.2)$ & $(0.2)$ \\
\hline \multirow[t]{2}{*}{ 8) Point of Shoal } & $5.33 \times 10^{5}$ & $17.3^{33}$ & $15.6^{33}$ & $11.7^{33}$ & $14.0^{33}$ & $16.0^{33}$ \\
\hline & & $(0.4)$ & $(0.3)$ & $(0.3)$ & $(0.3)$ & $(0.3)$ \\
\hline \multirow[t]{2}{*}{ 9) Cross Rock } & $1.48 \times 10^{5}$ & $15.0^{21}$ & $9.3^{21}$ & $9.3^{21}$ & $11.8^{21}$ & $14.6^{21}$ \\
\hline & & $(0.5)$ & $(0.4)$ & $(0.4)$ & $(0.4)$ & $(0.4)$ \\
\hline \multirow[t]{2}{*}{ 10) Shanty Rock } & $1.26 \times 10^{4}$ & $7.9^{7}$ & $6.4^{7}$ & $4.7^{7}$ & $3.3^{7}$ & $10.0^{7}$ \\
\hline & & $(1.2)$ & $(0.9)$ & $(0.4)$ & $(0.5)$ & $(1.2)$ \\
\hline \multirow[t]{2}{*}{ 11) Dry Lumps } & $2.54 \times 10^{4}$ & $19.9^{7}$ & $7.2^{7}$ & $13.4^{7}$ & $10.5^{7}$ & $16.1^{7}$ \\
\hline & & $(0.3)$ & $(0.8)$ & $(1.5)$ & (1.0) & $(0.7)$ \\
\hline \multirow[t]{2}{*}{ 12) Mulberry Point } & $1.96 \times 10^{5}$ & $5.9^{10}$ & $6.5^{8}$ & $5.0^{10}$ & $5.2^{10}$ & $7.2^{10}$ \\
\hline & & $(1.0)$ & $(0.9)$ & $(0.6)$ & $(0.7)$ & $(0.9)$ \\
\hline \multirow[t]{2}{*}{ 13) Swash } & $8.13 \times 10^{5}$ & $1.7^{22}$ & $0.7^{22}$ & $1.9^{22}$ & $2.3^{22}$ & $1.1^{22}$ \\
\hline & & $(0.1)$ & $(0.1)$ & $(0.1)$ & $(0.2)$ & $(0.1)$ \\
\hline \multirow[t]{2}{*}{ 14) Upper Jail Island } & $2.48 \times 10^{6}$ & $2.4^{30}$ & $0.6^{30}$ & $1.2^{30}$ & $0.7^{30}$ & $0.9^{30}$ \\
\hline & & $(0.1)$ & $(0.1)$ & $(0.1)$ & $(0.0)$ & $(0.1)$ \\
\hline \multirow[t]{2}{*}{ 15) Swash and Mud Slough } & $4.98 \times 10^{6}$ & $1.9^{30}$ & $1.7^{30}$ & $3.0^{30}$ & $3.0^{30}$ & $1.9^{30}$ \\
\hline & & $(0.1)$ & $(0.1)$ & $(0.1)$ & $(0.1)$ & $(0.1)$ \\
\hline \multirow[t]{2}{*}{ 16) Offshore Swash } & $2.59 \times 10^{6}$ & $3.1^{30}$ & $2.0^{30}$ & $1.7^{30}$ & $4.9^{30}$ & $2.2^{30}$ \\
\hline & & $(0.2)$ & $(0.1)$ & $(0.1)$ & $(0.2)$ & $(0.1)$ \\
\hline \multirow[t]{2}{*}{ 17) Lower Jail Island } & $2.55 \times 10^{6}$ & $0.6^{30}$ & $0.4^{30}$ & $0.5^{30}$ & $0.8^{30}$ & $0.7^{30}$ \\
\hline & & $(0.1)$ & $(0.1)$ & $(0.0)$ & $(0.1)$ & $(0.1)$ \\
\hline \multirow[t]{2}{*}{ 18) Offshore Jail Island } & $4.12 \times 10^{6}$ & $2.1^{30}$ & $0.9^{30}$ & $1.7^{30}$ & $2.0^{30}$ & $1.1^{30}$ \\
\hline & & $(0.1)$ & $(0.1)$ & $(0.1)$ & $(0.1)$ & $(0.1)$ \\
\hline \multirow[t]{2}{*}{ 19) Wreck Shoal } & $2.37 \times 10^{6}$ & $3.8^{30}$ & $5.2^{30}$ & $4.7^{30}$ & $3.5^{30}$ & $4.4^{30}$ \\
\hline & & $(0.1)$ & $(0.1)$ & $(0.2)$ & $(0.1)$ & $(0.1)$ \\
\hline \multirow[t]{2}{*}{ 20) Day's Point } & $1.21 \times 10^{6}$ & $3.0^{15}$ & $3.0^{15}$ & $1.9^{15}$ & $2.1^{15}$ & $3.9^{15}$ \\
\hline & & $(0.3)$ & $(0.2)$ & $(0.2)$ & $(0.2)$ & $(0.4)$ \\
\hline \multirow[t]{2}{*}{ 21) Hotel Rock } & $5.54 \times 10^{4}$ & $2.7^{7}$ & $2.6^{7}$ & $2.1^{7}$ & $4.3^{7}$ & $1.0^{7}$ \\
\hline & & $(0.6)$ & $(0.5)$ & $(0.5)$ & (0.6) & $(0.3)$ \\
\hline \multirow[t]{2}{*}{ 22) Snyder's Rock } & $3.75 \times 10^{4}$ & $0.9^{7}$ & $1.1^{6}$ & $1.9^{7}$ & $2.4^{7}$ & $1.6^{7}$ \\
\hline & & $(0.1)$ & $(0.1)$ & $(0.2)$ & $(0.3)$ & $(0.1)$ \\
\hline 23) Triangle Rock & $2.77 \times 10^{4}$ & $24.9^{7}$ & $7.9^{7}$ & $20.0^{20}$ & $19.7^{7}$ & $23.7^{7}$ \\
\hline & & $(1.1)$ & $(1.1)$ & $(0.7)$ & $(0.4)$ & $(0.8)$ \\
\hline
\end{tabular}

between 7.1 and $19.2 \mathrm{~L} \mathrm{~m}^{-2}$ with higher values to $19.9 \mathrm{~L} \mathrm{~m}^{-2}$ in 2002 and 2006. Remaining reefs were consistently less than $9 \mathrm{~L}$ $\mathrm{m}^{-2}$ with one exception, reef 19 in 1999 at $16.0 \mathrm{~L} \mathrm{~m}^{-2}$.

Brown shell values at reefs with the highest densities of total shell approached the total values indicating that little of the total shell was buried (Table 4, Fig. 5). At lower shell values the proportion of brown shell generally decreased which is evident in a comparison of brown shell to total shell ratio at reefs 3-8, 11-13 and 23 with a second suite of reefs 1, 2, 9 and 14-17, and a lower value suite consisting of reefs 10 and 19, and then 18-21 (Table 5,
ANOVA). When data are examined by year, 2006 exceeded all others in terms of proportion of brown shell to total shell. This may in part be associated with mortality of 4-y-old oysters originating in the 2002 recruitment (Table 1, Table 5). The death of even a modest number of these larger oysters contributes disproportionately to the brown shell reservoir because of the individual large size of a 4-y-old oyster.

The variance to mean ratios observed for both total shell volume (L, 1998 to 2006, Fig. 4B) and brown shell volume (L, 2002 to 2006, Fig. 4C) are between 1 and 10 . These relatively low 
TABLE 5.

Summary of statistics performed on the data from the James River. Significance levels were $P=\mathbf{0 . 0 5}$. Reef numbers correspond to those in Figure 1.

\begin{tabular}{|c|c|c|c|c|c|c|c|}
\hline Test & Response & Factor & Covariate & df & F/T Value & $P$ Value & $\begin{array}{l}\text { Multiple comparison } \\
\text { results (Fisher's test) }\end{array}$ \\
\hline \multirow[t]{3}{*}{ ANCOVA } & Oyster density & Reef & & 22 & 38.45 & $<0.001$ & $\begin{array}{l}\text { Reefs } 3,5,6>4,7,23>8 \\
9>\text { all others }\end{array}$ \\
\hline & & Year & & 8 & 72.10 & $<0.001$ & $2002>$ all others \\
\hline & & & Total volume & & 49.11 & $<0.001$ & \\
\hline \multirow[t]{3}{*}{ ANCOVA } & Oyster biomass & Reef & & 22 & 68.11 & $<0.001$ & $\begin{array}{l}\text { Reefs } 3,5,6>4,23>7 \\
\quad 8>9>\text { all others }\end{array}$ \\
\hline & & Year & & 4 & 109.56 & $<0.001$ & $\begin{array}{l}2005,2006>\text { all others: } \\
2005,2006>2000,2001 \\
2004>1998,1999,2003\end{array}$ \\
\hline & & & Total volume & & 47.20 & $<0.001$ & \\
\hline \multirow[t]{3}{*}{ ANCOVA } & Oyster density & Reef & & 22 & 6.17 & $<0.001$ & $\begin{array}{c}\text { Reefs } 3,6>4,5,7,23>8,9, \\
10,11,12>\text { all others }\end{array}$ \\
\hline & & Year & & 4 & 79.17 & $<0.001$ & $2002>$ all others \\
\hline & & & Brown volume & & 38.66 & $<0.001$ & \\
\hline \multirow[t]{3}{*}{ ANCOVA } & Oyster biomass & Reef & & 22 & 15.05 & $<0.001$ & $\begin{array}{l}\text { Reefs } 3,6>4,5,23>7, \\
\quad 8>\text { all others }\end{array}$ \\
\hline & & Year & & 4 & 134.80 & $<0.001$ & $\begin{array}{l}2005,2006>\text { all others: } \\
2004>2003\end{array}$ \\
\hline & & & Brown volume & & 28.87 & $<0.001$ & \\
\hline ANOVA & $\begin{array}{l}\text { Proportion brown } \\
\text { shell:total }\end{array}$ & Reef & & 22 & 39.00 & $<0.001$ & 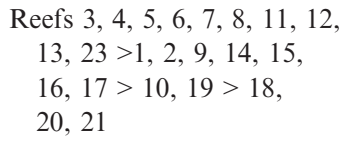 \\
\hline \multirow[t]{2}{*}{ Year } & & & & 4 & 12.92 & $<0.001$ & $\begin{array}{l}2006>\text { all others: } \\
2006>2002>2005\end{array}$ \\
\hline & & Reef X Year & & 88 & 2.68 & $<0.001$ & \\
\hline \multirow[t]{3}{*}{ ANOVA } & Total shell volume & Reef & & 22 & 114.41 & $<0.001$ & $\begin{array}{c}\text { Reefs } 5,6>3,4,7,8,23>1 \\
9,10,11,21>\text { all others }\end{array}$ \\
\hline & & Year & & 8 & 10.91 & $<0.001$ & $\begin{array}{l}2002>\text { all others: } 2002> \\
2000,2003>1998 \\
2004\end{array}$ \\
\hline & & Reef X Year & & 176 & 1.89 & $<0.001$ & \\
\hline
\end{tabular}

values indicate a somewhat random distribution in the shell resource with space and time when using either total shell or brown shell estimates as a barometer of habitat.

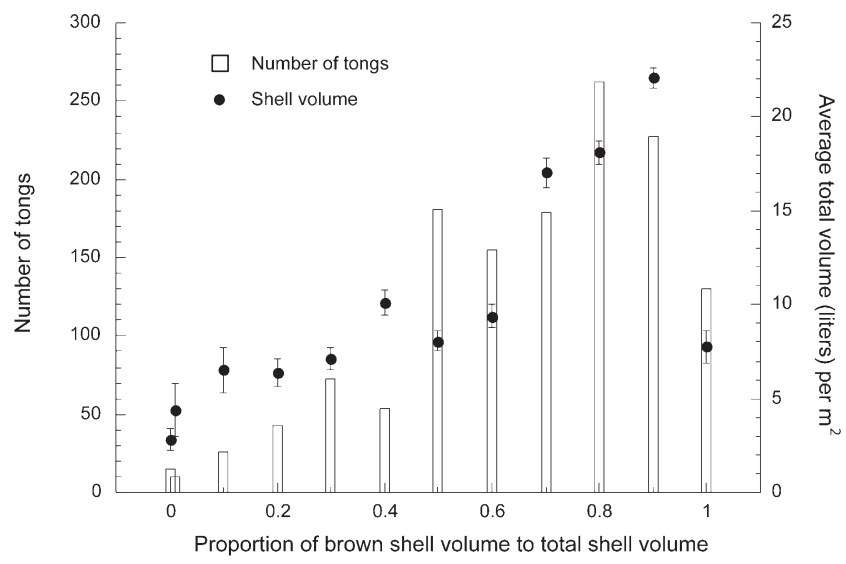

Figure 5. Proportion of brown shell volume (liters) to total shell volume (liters) compared with the number of tongs ( $\mathrm{Y} 1 \mathrm{axis}$ ) and the average total volume of shell meter-2 (Y2 axis).

\section{Relationship of Live Oyster Density, Biomass, and Shell} Volume Over Time

Both oyster density $\left(\mathrm{m}^{-2}\right)$ and biomass ( $\mathrm{g}$ dry tissue $\mathrm{m}^{-2}$ ) were significantly affected by year, reef, and total shell volume (Table 5, ANCOVA with total shell volume as a covariate) as well as brown shell volume (Table 5, ANCOVA with brown shell as a covariate). The total shell volume $\left(\mathrm{L} \mathrm{m}^{-2}\right)$ was significantly affected by both year (1998 to 2006) and reef (1-23, ANOVA, Table 5) and there was a significant interaction between year and reef (ANOVA, Table 5).

The previously described spatial variation in absolute density reflects consistency of recruitment with several years of postrecruit survival. Biomass per unit area values are less influenced by sporadic large numbers of early recruits, but the same patterns remain with reefs $3-9$ and 23 exhibiting highest biomass between 20 and $200 \mathrm{~g} \mathrm{~m}^{-2}$. These high density, high biomass per unit area populations occupy a very modest portion $(5.4 \%)$ of the total habitat area examined. Extensive areas support very few oysters in terms of either density or biomass, supporting the earlier suggestion of patchiness as indicated by the variance to mean ratios illustrated in Figure 4A. 
TABLE 6.

Total standing stock of oysters on the 23 reefs in the James River from 1993 through 2006. Data are presented as the total number of oysters for each reef/year combination with the sum for each year totaled across all 23 reefs in bold. " $X$ " indicates where data are not available for a particular year. The numbers for the individual reefs correspond to those in Figure 1. The bottom two rows give the percentage of the total for area and biomass for the two groups of reefs; high and medium standing stock reefs (reefs 3-9 and 23) and low standing stock reefs (all others) as discussed in the text.

\begin{tabular}{|c|c|c|c|c|c|c|c|}
\hline Station (reef name) & $\operatorname{Area}\left(m^{2}\right)$ & 1993 & 1994 & 1995 & 1996 & 1997 & 1998 \\
\hline 1) Upper Deep Water Shoal & $1.27 \times 10^{6}$ & $7.45 \times 10^{7}$ & $6.57 \times 10^{7}$ & $2.57 \times 10^{8}$ & $1.22 \times 10^{8}$ & $4.67 \times 10^{7}$ & $4.68 \times 10^{7}$ \\
\hline 2) Lower Deep Water Shoal & $8.07 \times 10^{4}$ & $5.24 \times 10^{6}$ & $1.82 \times 10^{6}$ & $1.50 \times 10^{6}$ & $4.44 \times 10^{5}$ & $5.30 \times 10^{5}$ & $5.75 \times 10^{5}$ \\
\hline 3) Upper Horsehead & $2.15 \times 10^{4}$ & $6.73 \times 10^{6}$ & $5.24 \times 10^{6}$ & $7.25 \times 10^{6}$ & $8.77 \times 10^{6}$ & $4.40 \times 10^{6}$ & $4.63 \times 10^{6}$ \\
\hline 4) Middle Horsehead & $1.77 \times 10^{5}$ & $3.61 \times 10^{7}$ & $4.17 \times 10^{7}$ & $8.40 \times 10^{7}$ & $9.45 \times 10^{7}$ & $5.58 \times 10^{7}$ & $5.52 \times 10^{7}$ \\
\hline 5) Lower Horsehead & $8.34 \times 10^{4}$ & $2.46 \times 10^{7}$ & $2.91 \times 10^{7}$ & $3.28 \times 10^{7}$ & $3.33 \times 10^{7}$ & $2.11 \times 10^{7}$ & $2.67 \times 10^{7}$ \\
\hline 6) Moon Rock & $1.34 \times 10^{4}$ & $3.63 \times 10^{6}$ & $5.57 \times 10^{6}$ & $5.27 \times 10^{6}$ & $3.92 \times 10^{6}$ & $2.50 \times 10^{6}$ & $3.23 \times 10^{6}$ \\
\hline 7) V-Rock & $3.07 \times 10^{5}$ & $5.46 \times 10^{7}$ & $6.68 \times 10^{7}$ & $8.50 \times 10^{7}$ & $1.10 \times 10^{8}$ & $8.09 \times 10^{7}$ & $1.05 \times 10^{8}$ \\
\hline 8) Point of Shoal & $5.33 \times 10^{5}$ & $6.82 \times 10^{6}$ & $1.05 \times 10^{8}$ & $1.56 \times 10^{8}$ & $1.25 \times 10^{8}$ & $1.04 \times 10^{8}$ & $8.81 \times 10^{7}$ \\
\hline 9) Cross Rock & $1.48 \times 10^{5}$ & $1.38 \times 10^{7}$ & $1.84 \times 10^{7}$ & $2.96 \times 10^{7}$ & $2.31 \times 10^{7}$ & $2.33 \times 10^{7}$ & $3.11 \times 10^{7}$ \\
\hline 10) Shanty Rock & $1.26 \times 10^{4}$ & $4.40 \times 10^{5}$ & $1.90 \times 10^{6}$ & $1.14 \times 10^{6}$ & $5.47 \times 10^{5}$ & $5.80 \times 10^{5}$ & $1.36 \times 10^{6}$ \\
\hline 11) Dry Lumps & $2.54 \times 10^{4}$ & $4.01 \times 10^{5}$ & $2.31 \times 10^{6}$ & $6.92 \times 10^{5}$ & $7.90 \times 10^{5}$ & $3.37 \times 10^{5}$ & $2.03 \times 10^{6}$ \\
\hline 12) Mulberry Point & $1.96 \times 10^{5}$ & $6.98 \times 10^{6}$ & $7.80 \times 10^{6}$ & $7.42 \times 10^{6}$ & $1.35 \times 10^{7}$ & $1.66 \times 10^{7}$ & $9.24 \times 10^{6}$ \\
\hline 13) Swash & $8.13 \times 10^{5}$ & $4.56 \times 10^{6}$ & $1.98 \times 10^{7}$ & $1.48 \times 10^{7}$ & $1.31 \times 10^{7}$ & $5.41 \times 10^{6}$ & $1.70 \times 10^{7}$ \\
\hline 14) Upper Jail Island & $2.48 \times 10^{6}$ & $2.75 \times 10^{7}$ & $4.65 \times 10^{7}$ & $2.68 \times 10^{7}$ & $3.18 \times 10^{7}$ & $3.10 \times 10^{7}$ & $5.09 \times 10^{7}$ \\
\hline 15) Swash and Mud Slough & $4.98 \times 10^{6}$ & $1.31 \times 10^{8}$ & $1.64 \times 10^{8}$ & $1.25 \times 10^{8}$ & $1.50 \times 10^{8}$ & $1.34 \times 10^{8}$ & $2.04 \times 10^{8}$ \\
\hline 16) Offshore Swash & $2.59 \times 10^{6}$ & $7.63 \times 10^{7}$ & $1.20 \times 10^{8}$ & $1.13 \times 10^{8}$ & $1.50 \times 10^{8}$ & $7.58 \times 10^{7}$ & $1.34 \times 10^{8}$ \\
\hline 17) Lower Jail Island & $2.55 \times 10^{6}$ & $3.44 \times 10^{7}$ & $3.00 \times 10^{7}$ & $3.44 \times 10^{7}$ & $2.91 \times 10^{7}$ & $2.50 \times 10^{7}$ & $4.81 \times 10^{7}$ \\
\hline 18) Offshore Jail Island & $4.12 \times 10^{6}$ & $4.20 \times 10^{7}$ & $9.22 \times 10^{7}$ & $3.59 \times 10^{7}$ & $3.87 \times 10^{7}$ & $2.96 \times 10^{7}$ & $7.11 \times 10^{7}$ \\
\hline 19) Wreck Shoal & $2.37 \times 10^{6}$ & $2.06 \times 10^{7}$ & $3.79 \times 10^{7}$ & $1.85 \times 10^{7}$ & $1.26 \times 10^{7}$ & $6.28 \times 10^{6}$ & $3.23 \times 10^{7}$ \\
\hline 20) Day's Point & $1.21 \times 10^{6}$ & $\mathrm{X}$ & $3.29 \times 10^{7}$ & $1.02 \times 10^{7}$ & $1.01 \times 10^{7}$ & $1.35 \times 10^{7}$ & $3.33 \times 10^{7}$ \\
\hline 21) Hotel Rock & $5.54 \times 10^{4}$ & $\mathrm{X}$ & $3.76 \times 10^{6}$ & $8.00 \times 10^{5}$ & $1.92 \times 10^{6}$ & $1.92 \times 10^{6}$ & $4.09 \times 10^{6}$ \\
\hline 22) Snyder's Rock & $3.75 \times 10^{4}$ & $\mathrm{X}$ & $1.33 \times 10^{6}$ & $6.80 \times 10^{5}$ & $9.96 \times 10^{5}$ & $7.44 \times 10^{5}$ & $1.30 \times 10^{6}$ \\
\hline 23) Triangle Rock & $2.77 \times 10^{4}$ & $\mathrm{X}$ & $4.28 \times 10^{6}$ & $5.05 \times 10^{6}$ & $8.17 \times 10^{6}$ & $5.59 \times 10^{6}$ & $5.48 \times 10^{6}$ \\
\hline TOTAL & $2.41 \times 10^{7}$ & $5.70 \times 10^{8}$ & $9.05 \times 10^{8}$ & $1.05 \times 10^{9}$ & $9.83 \times 10^{8}$ & $6.85 \times 10^{8}$ & $9.76 \times 10^{8}$ \\
\hline$\%$ of total (reefs 3-9 and 23) & 5.4 & 25.7 & 30.6 & 38.5 & 41.4 & 43.4 & 32.7 \\
\hline$\%$ of total (all others) & 94.6 & 74.3 & 69.4 & 61.5 & 58.6 & 56.6 & 67.3 \\
\hline
\end{tabular}

Oyster density and biomass do not track temporally as they do spatially because of changes in recruitment patterns over time. 2002 was an exceptional recruitment year with high density observed across the entire system and densities $>400$ oysters $\mathrm{m}^{-2}$ recorded at reefs $3,5,6,7$. and 23 . Only four other records of densities $>400$ oysters $\mathrm{m}^{-2}$ were recorded for all reefs for the entire fourteen-year period (Table 1).

Oyster density was significantly higher in 2002 than in all other years (Table 5, ANCOVA with either total shell or brown shell volume as covariate, $P<0.05)$ and oyster biomass was significantly higher in 2005 and 2006 than in all other years (Table 5, ANCOVA with either total shell or brown shell volume as covariate, $P<0.05) .2002$ had the highest oyster density compared with all other years, but was among the lowest in oyster biomass. High density, when coupled with low biomass, is indicative of a year of good recruitment, which was the case during 2002 (Southworth et al. 2003). The year 2002 was characterized by low river flows, which resulted in higher than normal salinities. Increasing recruitment (spatfall) and overall oyster density are often associated with higher salinity in the James River. An increase in biomass is expected in the 1-2 y after a year of good recruitment, as was the case between 1995 (high density) and 1996 (high biomass) and 1999 (high density) and 2001 (high biomass). This was, however, not the case between 2002 (high density) and 2003 (low biomass). Extended low salinities through the summer of 2003 and the added stress of fresh water from Hurricane Isabel in September 2003 (indicated on Fig. 3) resulted in a notable mortality as demonstrated by the large number of boxes observed during sampling at many of the stations, especially those in the upper reaches of the James River. Recruitment was sustained in 2004 and 2005 with modest mortality, resulting in the highest biomass values for the entire study period being recorded for 11 reefs in 2005 (reefs 3-7, 9, 16, 18, 19, 21, and 23) and 5 reefs in 2006 (reefs 8, 10-12, and 20). Mortality data is examined in detail in a later section of this report.

The proportion of brown shell to total shell volume within individual patent tong grabs (sampling unit) was significantly affected by both year and reef (ANOVA, Table 5) and there was a significant interaction between year and reef (ANOVA, Table 5). The highest brown shell volumes in the 2002-2006 period were observed in 2006 (Fisher's test, Table 5); these values were significantly higher than those observed in either 2002 or 2005 (Fisher's test, Table 5).

The relationship between oyster shell length $(\mathrm{mm})$ and oyster wet shell weight $(\mathrm{g})$ for 73 James River oysters was described using a power equation:

$$
\text { Wet shell weight } \begin{aligned}
(\mathrm{g}) & =0.002374^{*} \text { Shell length }(\mathrm{mm})^{2.21} ; \\
\mathrm{R}^{2} & =0.64
\end{aligned}
$$

This relationship was used to estimate the amount of live shell (g) observed in each patent tong on the basis of the available live oyster and box demographics. A volumetric conversion was 
Oyster Population Biology

\begin{tabular}{|c|c|c|c|c|c|c|c|}
\hline 1999 & 2000 & 2001 & 2002 & 2003 & 2004 & 2005 & 2006 \\
\hline $3.18 \times 10^{7}$ & $3.10 \times 10^{7}$ & $4.07 \times 10^{7}$ & $2.37 \times 10^{8}$ & $1.15 \times 10^{7}$ & $9.38 \times 10^{6}$ & $4.82 \times 10^{6}$ & $2.01 \times 10^{7}$ \\
\hline $4.44 \times 10^{5}$ & $4.54 \times 10^{5}$ & $1.45 \times 10^{6}$ & $8.95 \times 10^{6}$ & $3.33 \times 10^{5}$ & $6.29 \times 10^{5}$ & $2.74 \times 10^{5}$ & $1.69 \times 10^{6}$ \\
\hline $2.61 \times 10^{6}$ & $6.04 \times 10^{6}$ & $6.90 \times 10^{6}$ & $1.66 \times 10^{7}$ & $9.88 \times 10^{6}$ & $8.68 \times 10^{6}$ & $6.56 \times 10^{6}$ & $5.29 \times 10^{6}$ \\
\hline $4.10 \times 10^{7}$ & $2.98 \times 10^{7}$ & $4.48 \times 10^{7}$ & $5.85 \times 10^{7}$ & $5.96 \times 10^{7}$ & $3.04 \times 10^{7}$ & $4.66 \times 10^{7}$ & $2.72 \times 10^{7}$ \\
\hline $2.80 \times 10^{7}$ & $2.55 \times 10^{7}$ & $2.92 \times 10^{7}$ & $4.06 \times 10^{7}$ & $2.81 \times 10^{7}$ & $2.39 \times 10^{7}$ & $2.85 \times 10^{7}$ & $1.85 \times 10^{7}$ \\
\hline $3.51 \times 10^{6}$ & $4.31 \times 10^{6}$ & $4.48 \times 10^{6}$ & $8.07 \times 10^{6}$ & $6.06 \times 10^{6}$ & $4.56 \times 10^{6}$ & $4.22 \times 10^{6}$ & $3.91 \times 10^{6}$ \\
\hline $6.42 \times 10^{7}$ & $8.34 \times 10^{7}$ & $6.29 \times 10^{7}$ & $1.34 \times 10^{8}$ & $7.66 \times 10^{7}$ & $8.48 \times 10^{7}$ & $6.65 \times 10^{7}$ & $5.32 \times 10^{7}$ \\
\hline $5.97 \times 10^{7}$ & $7.15 \times 10^{7}$ & $5.79 \times 10^{7}$ & $1.94 \times 10^{8}$ & $7.88 \times 10^{7}$ & $7.61 \times 10^{7}$ & $7.66 \times 10^{7}$ & $7.64 \times 10^{7}$ \\
\hline $2.79 \times 10^{7}$ & $2.27 \times 10^{7}$ & $2.24 \times 10^{7}$ & $3.50 \times 10^{7}$ & $1.60 \times 10^{7}$ & $2.36 \times 10^{7}$ & $2.19 \times 10^{7}$ & $2.20 \times 10^{7}$ \\
\hline $9.24 \times 10^{5}$ & $3.18 \times 10^{5}$ & $5.13 \times 10^{5}$ & $4.87 \times 10^{5}$ & $4.19 \times 10^{5}$ & $1.57 \times 10^{6}$ & $5.45 \times 10^{5}$ & $1.22 \times 10^{6}$ \\
\hline $3.17 \times 10^{6}$ & $1.51 \times 10^{6}$ & $8.77 \times 10^{5}$ & $4.63 \times 10^{6}$ & $1.22 \times 10^{6}$ & $7.34 \times 10^{6}$ & $4.09 \times 10^{6}$ & $4.20 \times 10^{6}$ \\
\hline $7.84 \times 10^{5}$ & $1.65 \times 10^{6}$ & $4.88 \times 10^{6}$ & $2.96 \times 10^{7}$ & $1.05 \times 10^{7}$ & $1.18 \times 10^{7}$ & $9.78 \times 10^{6}$ & $1.09 \times 10^{7}$ \\
\hline $1.24 \times 10^{7}$ & $9.21 \times 10^{6}$ & $1.12 \times 10^{7}$ & $3.97 \times 10^{7}$ & $4.22 \times 10^{6}$ & $1.43 \times 10^{7}$ & $9.52 \times 10^{6}$ & $5.12 \times 10^{6}$ \\
\hline $3.19 \times 10^{7}$ & $9.65 \times 10^{6}$ & $1.34 \times 10^{7}$ & $1.25 \times 10^{8}$ & $1.37 \times 10^{7}$ & $2.65 \times 10^{7}$ & $1.06 \times 10^{7}$ & $1.41 \times 10^{7}$ \\
\hline $1.14 \times 10^{8}$ & $1.15 \times 10^{8}$ & $2.97 \times 10^{7}$ & $1.19 \times 10^{8}$ & $7.95 \times 10^{7}$ & $2.08 \times 10^{8}$ & $1.15 \times 10^{8}$ & $7.12 \times 10^{7}$ \\
\hline $1.09 \times 10^{8}$ & $4.94 \times 10^{7}$ & $1.10 \times 10^{8}$ & $1.37 \times 10^{8}$ & $4.73 \times 10^{7}$ & $6.28 \times 10^{7}$ & $2.09 \times 10^{8}$ & $4.59 \times 10^{7}$ \\
\hline $4.62 \times 10^{7}$ & $2.45 \times 10^{7}$ & $1.18 \times 10^{7}$ & $1.24 \times 10^{7}$ & $5.85 \times 10^{6}$ & $1.15 \times 10^{7}$ & $6.36 \times 10^{6}$ & $6.36 \times 10^{6}$ \\
\hline $5.12 \times 10^{7}$ & $1.21 \times 10^{7}$ & $2.22 \times 10^{7}$ & $2.51 \times 10^{7}$ & $1.21 \times 10^{7}$ & $1.35 \times 10^{8}$ & $7.08 \times 10^{7}$ & $3.54 \times 10^{7}$ \\
\hline $2.17 \times 10^{7}$ & $8.36 \times 10^{6}$ & $2.41 \times 10^{7}$ & $8.54 \times 10^{7}$ & $4.21 \times 10^{7}$ & $1.85 \times 10^{8}$ & $9.96 \times 10^{7}$ & $4.14 \times 10^{7}$ \\
\hline $1.25 \times 10^{7}$ & $9.55 \times 10^{6}$ & $1.24 \times 10^{7}$ & $3.79 \times 10^{7}$ & $1.10 \times 10^{7}$ & $2.50 \times 10^{7}$ & $1.74 \times 10^{7}$ & $1.98 \times 10^{7}$ \\
\hline $1.29 \times 10^{6}$ & $2.38 \times 10^{5}$ & $2.21 \times 10^{6}$ & $2.19 \times 10^{6}$ & $1.39 \times 10^{6}$ & $1.62 \times 10^{6}$ & $1.79 \times 10^{6}$ & $1.89 \times 10^{5}$ \\
\hline $8.03 \times 10^{4}$ & $3.75 \times 10^{4}$ & $8.57 \times 10^{4}$ & $1.39 \times 10^{5}$ & $4.37 \times 10^{4}$ & $1.12 \times 10^{6}$ & $4.38 \times 10^{5}$ & $2.36 \times 10^{5}$ \\
\hline $7.11 \times 10^{6}$ & $5.66 \times 10^{6}$ & $3.70 \times 10^{6}$ & $1.39 \times 10^{7}$ & $2.80 \times 10^{6}$ & $7.28 \times 10^{6}$ & $6.28 \times 10^{6}$ & $4.75 \times 10^{6}$ \\
\hline $6.71 \times 10^{8}$ & $5.22 \times 10^{8}$ & $5.18 \times 10^{8}$ & $1.37 \times 10^{9}$ & $5.19 \times 10^{8}$ & $9.61 \times 10^{8}$ & $8.17 \times 10^{8}$ & $4.89 \times 10^{8}$ \\
\hline 34.9 & 47.7 & 44.8 & 36.7 & 53.5 & 27.0 & 31.5 & 43.2 \\
\hline 65.1 & 52.3 & 55.2 & 63.3 & 46.5 & 73.0 & 68.5 & 56.8 \\
\hline
\end{tabular}

also estimated by weighing $1 \mathrm{~L}$ shell samples, including a range of shell types from whole shells to shell hash, collected from five reefs in the James River in November 2006.

$$
1 \mathrm{~L} \text { of wet James River shell }=587.3 \mathrm{~g} \pm 22.6 \mathrm{~g}
$$

At first this relationship may appear spurious in that the specific gravity is $<1.00$; however, it is important to note that when shell is collected en mass and volume is assessed in a bucket or similar vessel much of the volume is open space between the collected valves and fragments. The specific gravity of the sample will only approach that of calcium carbonate when the entire shell collection is crushed. Data collected here is for intact valves and thus represents a working field correction for shell volume (L) to shell wet weight $(\mathrm{g})$ conversions.

\section{Description of the Oyster Resource: Standing Stock and Total Biomass}

Oyster standing stock in numbers and biomass was estimated by multiplying average oyster densities and biomass per unit area (Tables 1 and 2) by the reef area. Table 6 shows year and reef specific as well as total (sum of all reefs) estimated oyster-standing stock for the period 1993 to 2006, with the exception of reefs 20 23 that are reported for 1994 to 2006. Table 7 gives corresponding oyster biomass for the 1998 to 2006 period. The total number of oysters in the surveyed area varied between $4.89 \times 10^{8}$ in 2006 and $1.37 \times 10^{9}$ in 2002 . Seven years had values in the range 4.89 $6.68 \times 10^{8}$. Recruitment events in 1994 to $1996,1998,2002,2004$ and 2005 increased the standing stock to levels in the range $8.17 \times 10^{8}$ to $1.37 \times 10^{9}$. The distribution of the population by area is a reflection of density in that reefs 3 through 9 and 23 inclusive have a total area of $1.42 \times 10^{6} \mathrm{~m}^{2}$, or $5.4 \%$ of the total area, yet they contain between 25.7 and $55.5 \%$ by number. Total biomass varied between $1.07 \times 10^{8} \mathrm{~g}$ and $3.31 \times 10^{8} \mathrm{~g}(107 \mathrm{and}$ 331 metric tonnes) in 2003 and 2005, respectively. Biomass decreased each year from $1998\left(2.92 \times 10^{8} \mathrm{~g}\right)$ through 2003 (292-107 metric tons). Note that the 2002 to 2003 decrease in biomass occurred despite the observed increase in density (Table 1) during the same period. As the 2002 y class survived and grew, a high biomass value was observed in 2005. Increasing mortality in this year class during 2006 resulted in a subsequent biomass decrease to $2.22 \times 10^{8} \mathrm{~g}$ (222 metric tons). As with the total numbers of oysters present, the biomass was concentrated in reefs 3-9 inclusive and 23 containing between 35.8 and $54.8 \%$ of the total oyster population biomass.

Observed decreases in density and biomass include natural mortality, commercial harvest and relocation of seed oysters as part of fishery management, by VMRC, for "grow out" and eventual harvest at a later date. Records of direct harvest and relocation are of interest to assess the relative roles of natural and fishery related mortality. VMRC records of harvest and movement are reported in bushels. Removal and relocation of seed is reflected in sequential year and reef specific population estimates in Tables 6 and 7. Year specific transfers of seed oysters for management (repletion) are described in Table 8 and 
MAnN ET AL.

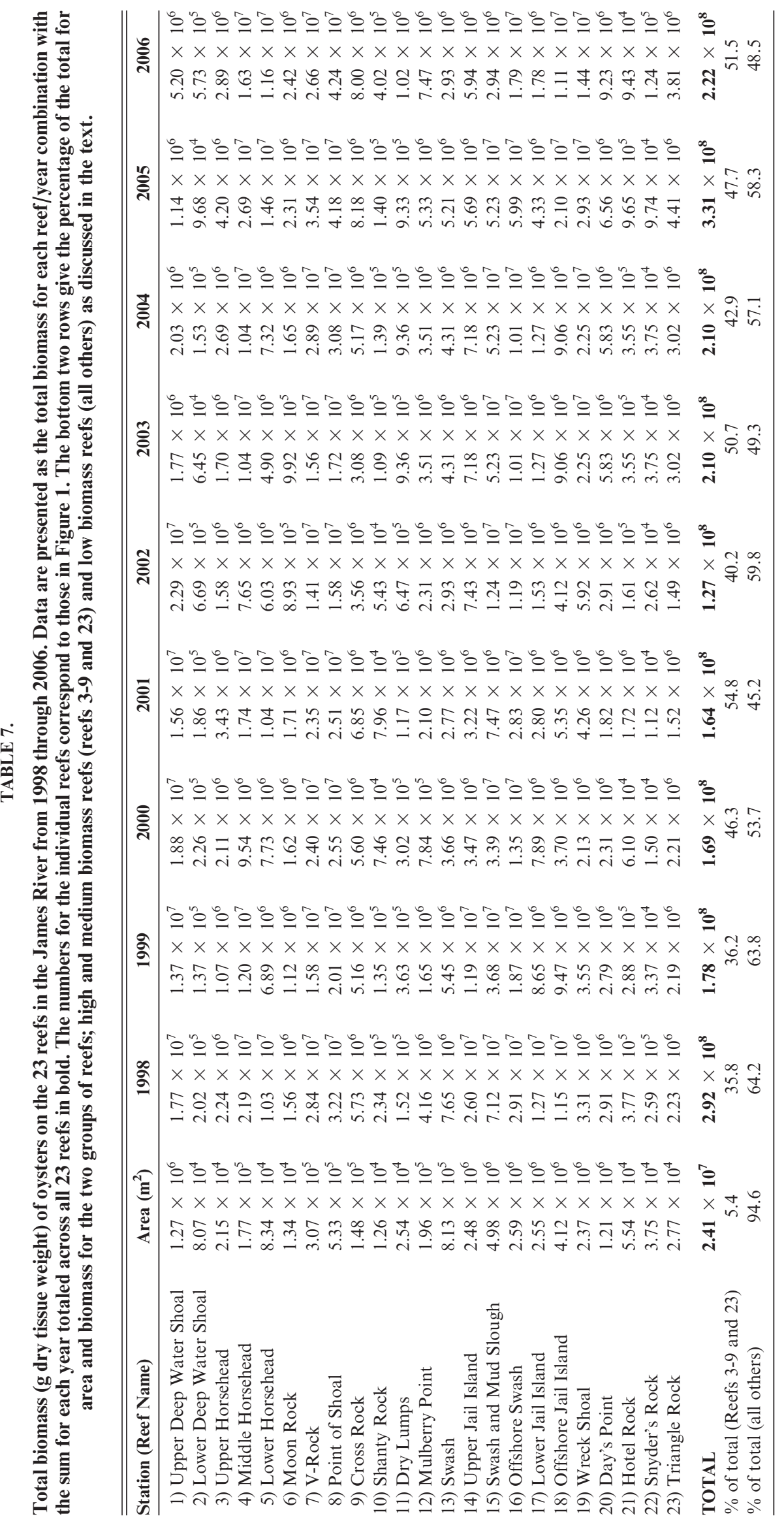




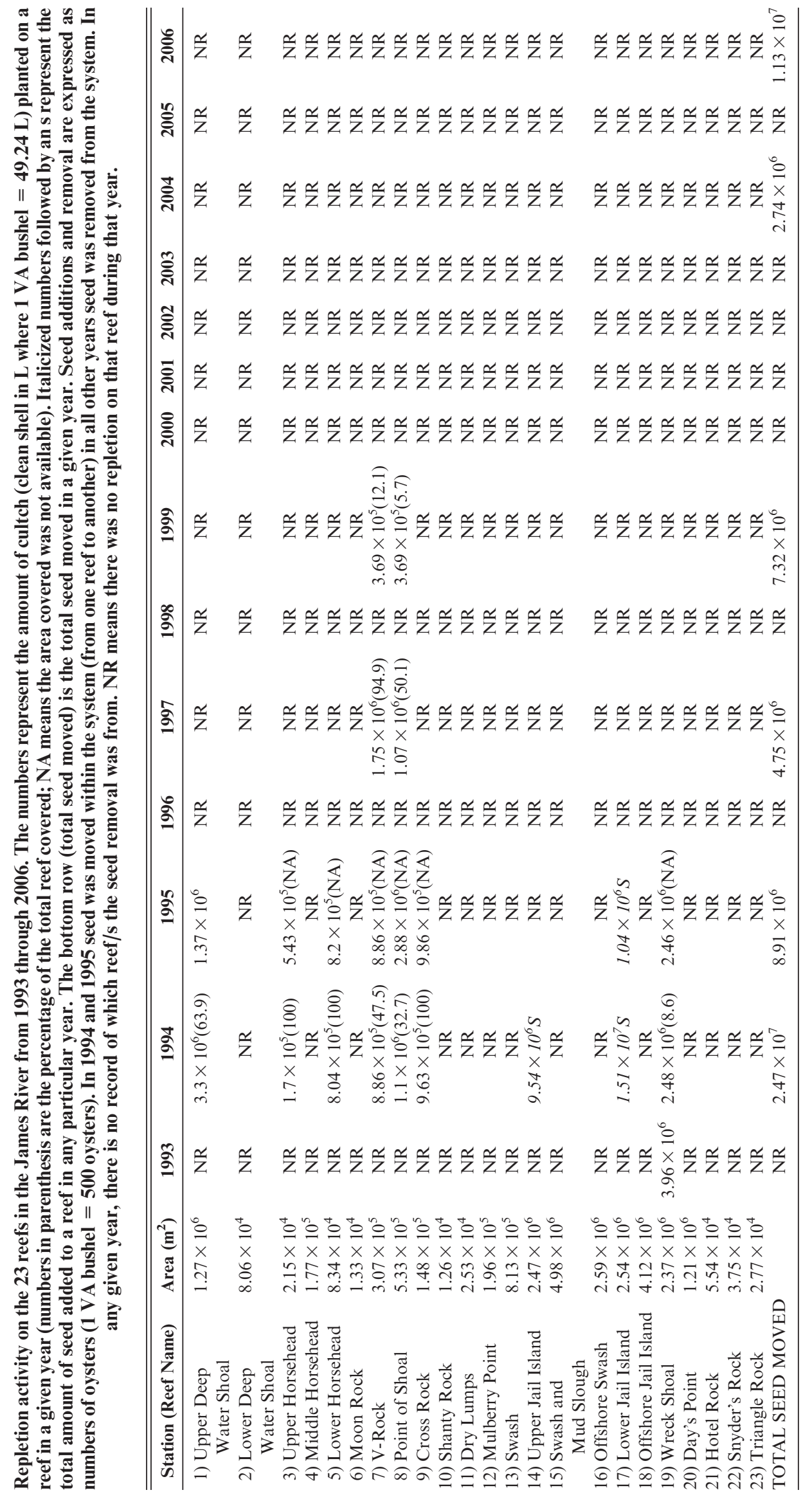




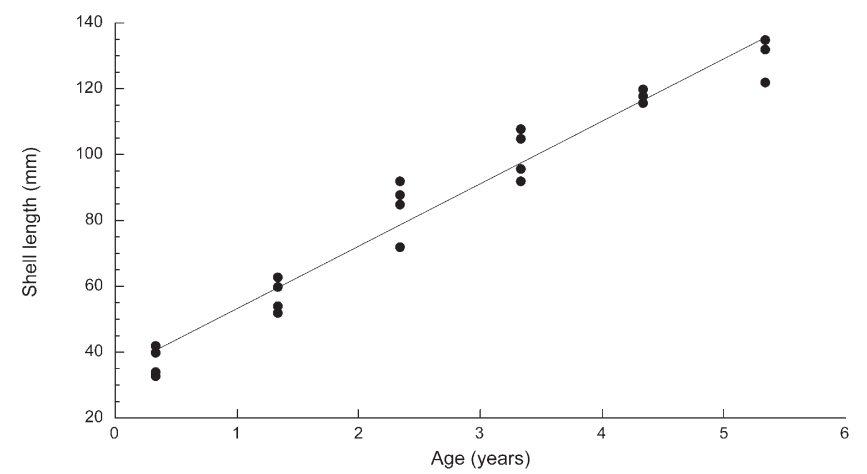

Figure 6. Estimation of oyster age-at-length based on data from all reefs with densities exceeding 100 oysters $\mathrm{m}^{-2}$. from 2004 to 2006 . The linear model is $y=30.22+21.6 x ; R^{2}=0.93$.

have been converted to total numbers of oysters from bushels for comparison with the standing stock estimates given in Table 6. In general, these relocations are a very modest proportion of the total oyster population and have limited impact on reef specific populations over the course of the study. Modest movement of oysters between public grounds and private leases also occur within the river; however, we have no data available on such movements. Table 8 also gives information on shell additions over the study period as part of management. As with oyster relocations, overall shell additions in the study area have been relatively modest throughout the course of the study.

\section{Estimation of Oyster Age at Length}

The linear fit in Figure $6(\mathrm{y}=\mathrm{mx}+\mathrm{b}$ where $\mathrm{y}=$ length $(\mathrm{mm})$, $\mathrm{m}=21.6 \pm 1.48, \mathrm{x}=$ age $(\mathrm{yr})$ and $\mathrm{b}=30.22 \pm 3.17)$ has both $\mathrm{a}$ remarkable $n$ value for the originating data set (81,369 individual oysters measured 2003 to 2006) and $R^{2}(0.93)$. Using a July 1 birth date and noting that current data is for a fall survey, then lengths on November 1 represent ages of $0.33,1.33$, and so on with annual increments, although for clarity throughout the rest of the text these ages will be referred to as 0-, 1-, 2-, 3-, and 4-y- olds, respectively. Corresponding shell lengths are $37.3 \mathrm{~mm}$ at $0 \mathrm{y}, 58.9 \mathrm{~mm}$ at $1 \mathrm{y}, 80.5 \mathrm{~mm}$ at $2 \mathrm{y}, 102.1 \mathrm{~mm}$ at $3 \mathrm{y}$, and 123.7 $\mathrm{mm}$ at $4 \mathrm{y}$ This age-at-length relationship was used to recast the length demographic as an age demographic and to estimate age specific mortality as described earlier.

The current estimates of age-at-length are considerably higher than those reported previously by Mann and Evans (1998) for oysters collected at Horsehead Reef (reef 3). Oysters in this location grow at the lower end of the salinity cline in the James River and are thus expected to have lower growth rates. In addition, Mann and Evans (2004) used an oscillating von Bertalanffy estimator which, unlike the linear estimator of Figure 6 , describes an exponential age-at-length relationship. Whereas the growth rates reported herein are generally higher than those reported in Kraeuter et al. (2007) for natural oyster populations in the Delaware Bay, they are nearly identical to growth rates calculated by Harding et al. $\left(2008, y=21.57 x+14.43, R^{2}=0.94\right.$, $n=3,315$ oysters examined) for three modern James River oyster populations at or below the mouth of the Warwick River.

\section{The Stock-recruitment Relationship}

Figure 7 presents a stock (year $\mathrm{t}$ ) versus recruit (year $\mathrm{t}+1$ ) plot for the entire river system for 1998 to 2006 data inclusive. Oysters $<47.5 \mathrm{~mm}$ SL were considered recruits, whereas those $47.5 \mathrm{~mm}$ or larger were considered adults in agreement with the age-at-length relationship in Figure 6. Whereas individual reefs provide a descriptor of habitat distribution, and cohort recruitment and survival over time, the stock recruit curve describes the river-wide stock with an assumption of no immigration, and where all larvae have the opportunity to recruit within the river system. When attempts were made to fit either Beverton-Holt or Ricker models to the raw or logarithm transformed data, these generated coefficients of determination of $<0.10$ and at least one model coefficient of either 0 or so large as to be nonsense. We can identify no defensible stock to recruit relationship in this population.

In all years but 2005 and 2006 the number of recruits exceeded the number of stock. This "replacement ratio" of $>1.0$

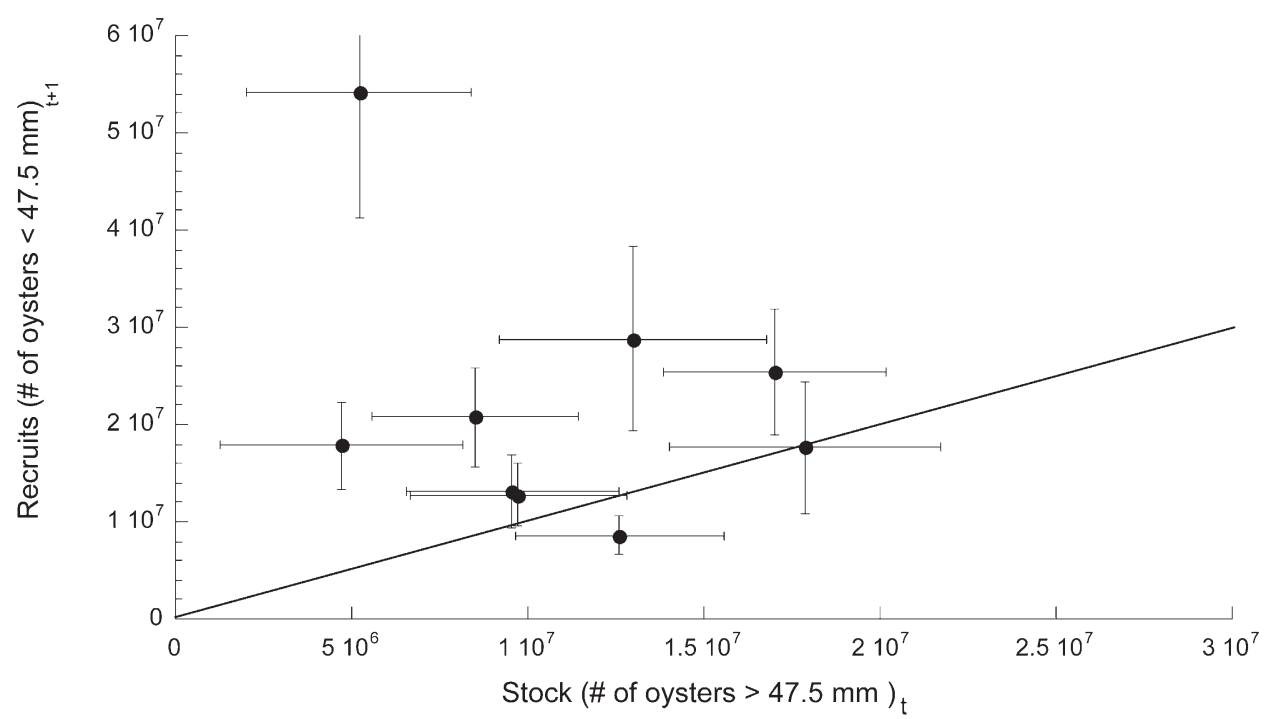

Figure 7. Standing stock (year t) versus recruits (year $\mathrm{t}+1$ ) for the entire river system for 1998 to 2006 data inclusive. Oysters $<47.5 \mathrm{~mm}$ SL were considered recruits. Oysters $47.5 \mathrm{~mm}$ shell length or larger were considered adults (see text). 


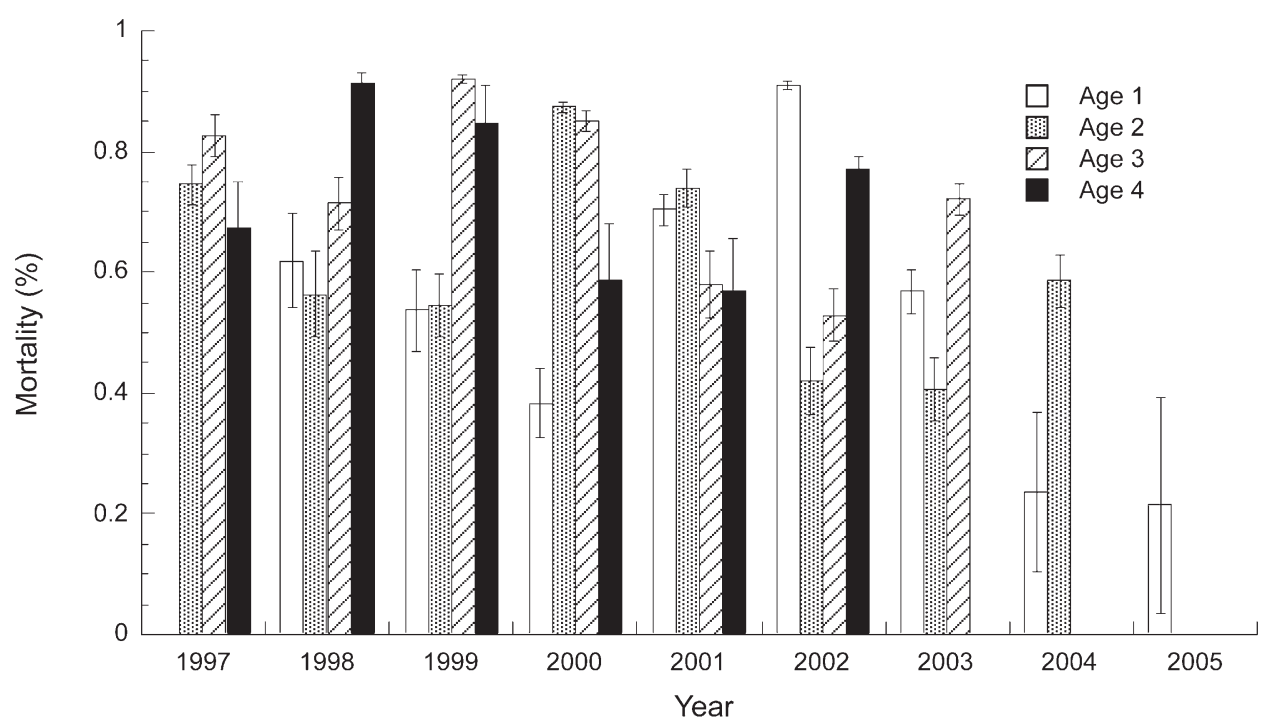

Figure 8. Age-specific oyster mortality by year class from 1997 to 2005.

would typically suggest an expanding population over time; however, the high mortalities (Fig. 8, discussed later in this section) in age classes $2-5$ result in stock size (in absolute numbers) below that of one year of recruits. The stock-recruit plot (Fig. 7) is dominated by a single recruitment event in 2002 at $5.42 \times 10^{7}$ concurrent with the second lowest standing stock, $5.22 \times 10^{6}$, in the entire study period.

\section{Mortality}

The age-at-length relationship (Fig. 6) was used to recast length demographics as age frequencies within each reef and year. As an example, Table 9 describes the time series for reef 6 , a high-density reef, for the period 1998 to 2006. Where the originating length data is in $5 \mathrm{~mm}$ bins (1998 to 2002), it has been proportionately divided among the year classes. Both the variation and intensity of recruitment rate is evident. Recruitment rates varied between 64 (2005) and $538 \mathrm{~m}^{-2}$ (2002) over the study period as recorded in the fall after the actual settlement events. Mortality rates were also high as illustrated by a comparison between the 0 and 1 y age values within a cohort. High recruitment does not necessarily translate to a higher density of 3- and 4-y-old oysters. Survival to these ages is typically very low, on the order of a few percent.
A summary of age-specific proportional mortality (Eq. 2) by year class is given in Figure 8. Mean mortality values for age 1 range from a low of $0.2-0.4$ in 2000, 2004, and 2005 to highs in excess of 0.7 in 2001 and 2002. It is important to reiterate the probability of underestimation of mortality in this age category. Age 2 mean values range from lows of 0.41 in 2002 and 2003 to highs exceeding 0.75 in 1997, 2000, and 2001. Age 3 and 4 proportional mortalities generally exceeded mean values of 0.6 with highest values approaching 0.95 for Age 4 in 1998 and Age 3 in 1999 (Fig. 8).

Comparison of Demographic Based Mortality Rates to Box Mortality

Estimates of age-specific mortality rates (Eq. 2) were compared with the mortality rates calculated from box counts (Eq. 3) based on the assignments of boxes to year classes as described earlier. Figure 9 illustrates data for all reefs with densities $>100$ oysters $\mathrm{m}^{-2}$ (all ages included) for the period 1998 to 2002. Only reefs with densities greater than 100 oysters $\mathrm{m}^{-2}$ were used in this calculation because they provided the most stable estimates over the time period of interest in that there were enough individuals to follow between years for these sites.

A bias towards underestimation of mortality rate is evident for box count derived rates at all ages, but this is especially large

TABLE 9.

An example of age demographic change over time for one high density reef (reef 6) from 1998 to 2006 after recasting length demographics using Figure 6 . The progression over time of a year class can be followed by moving diagonally across the table. The italicized values are the 1998 and 2001 y classes. The bold values are the 1999 and 2002 y classes.

\begin{tabular}{lrrrrrrrrr}
\hline \hline Age & $\mathbf{1 9 9 8}$ & $\mathbf{1 9 9 9}$ & $\mathbf{2 0 0 0}$ & $\mathbf{2 0 0 1}$ & $\mathbf{2 0 0 2}$ & $\mathbf{2 0 0 3}$ & $\mathbf{2 0 0 4}$ & $\mathbf{2 0 0 5}$ & $\mathbf{2 0 0 6}$ \\
\hline 0.33 & 79.1 & $\mathbf{1 6 6 . 8}$ & 166.9 & 160.8 & $\mathbf{5 3 8 . 6}$ & 390.9 & 170.1 & 64.0 & 64.1 \\
1.33 & 94.5 & 56.0 & $\mathbf{1 0 8 . 7}$ & 114.3 & 45.6 & $\mathbf{5 1 . 1}$ & 135.6 & 162.3 & 112.1 \\
2.33 & 57.0 & 33.4 & 37.2 & $\mathbf{4 9 . 2}$ & 14.9 & 10.1 & $\mathbf{2 8 . 1}$ & 77.6 \\
3.33 & 9.7 & 6.8 & 9.6 & 10.4 & $\mathbf{4 . 5}$ & 1.8 & 6.4 & $\mathbf{1 1 . 2}$ & 25.9 \\
4.33 & 1.5 & 0.1 & 0.6 & 0.5 & 0.6 & $\mathbf{0 . 2}$ & 0.9 & 0.9 & $\mathbf{3 . 0}$ \\
total & 241.8 & 263.1 & 322.8 & 335.2 & 604.1 & 454.0 & 341.1 & 316.0 & 292.3 \\
\hline
\end{tabular}




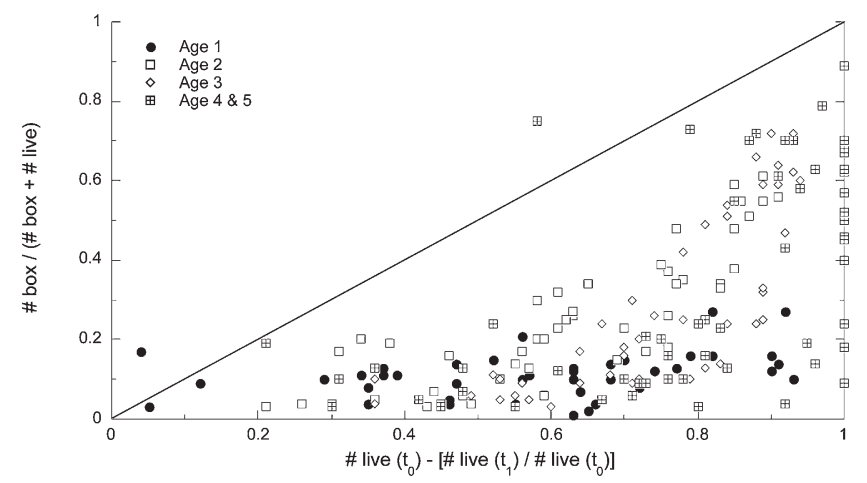

Figure 9. A comparison of age-specific mortality rate based on live oyster counts ( $x$ axis) and box counts ( $y$ axis) - see text for additional details. The diagonal line represents a 1:1 relationship where the mortality rate from one method equals the rate from the other.

for the first year of life (Fig. 9). This discrepancy decreases with increasing age but remains a source of concern for previously reported data. Only two data points in the entire record, both for 4-y-old classes, give higher mortality rates by box counts than the age-demographic method.

\section{Disease Impacts on Mortality}

The James River oyster resource is subject to periodic epizootics of both Dermo (Perkinus marinus) and MSX (Haplosporidium nelsoni). The prevalence and intensity of both are related to salinity and temperature (Andrews 1984; 1988, Ford \& Haskin 1988, Ford 1992). Low winter salinities may expulse MSX; however, Dermo can remain as very low level infections in the presence of low salinities (Burreson \& Ragone Calvo 1996). The presence of such infections, especially when winter salinities are high, can accelerate epizootics in the following spring and summer. Highest mid to late summer weighted prevalence values for Dermo in the current time series were observed in 1994, 1995, and 1999 through 2002 (Fig. 10). These years were all preceded by winters in which salinity was in excess of 8-9 ppt at reef 19 (Fig. 3). The 1995 to 1996 winter was unusually cold with January 1996 temperatures approaching $1{ }^{\circ} \mathrm{C}$ (Fig. 2). The multiyear period between 1999 and 2002 fostered severe epizootic conditions by 2002, despite short but cold winter events in 1999 to 2000 (approaching $2^{\circ} \mathrm{C}$ ) and 2000 to $2001\left(3^{\circ} \mathrm{C}\right)$ with a decrease in biomass associated with relatively high mortality (note the trends in Tables 2 and 7) despite an increase in recruitment (Tables 1 and 6). The 2002 to 2003 winter was cold with minima in the $1.0^{\circ} \mathrm{C}$ to $2.0^{\circ} \mathrm{C}$ range (Fig. 2). The following 2003 through mid 2005 period was marked by increased river flow, decreased salinity, and a warm $\left(6^{\circ} \mathrm{C}\right)$ winter in 2003 to 2004 . Winter salinities fell to $7 \mathrm{ppt}$ at reef 19 whereas summer salinity at the same site exceeded 9 ppt for 4 and 5 mo respectively in 2003 and 2004 (Fig. 3). Survival during this period improved (Tables 2 and 7) although recruitment was modest (Tables 1 and 6). Although winter salinities in 2004 to 2005 and 2005 to 2006 fell well below 9 ppt at reef 19 , summer salinities exceeded $12-13$ ppt for several months. The 2005 to 2006 winter was warm with a minimum temperature of approximately $7^{\circ} \mathrm{C}$. Weighted prevalence increased compared with 2003 to 2004 as did mortality (Tables 1 and 6, Fig. 8).

MSX weighted prevalence exceeded 1.0 in 1993, 1994, 1997 (by a very small margin) and, at the end of the 1999 to 2002 dry period, in 2002. The low salinities observed between the end of 2002 through 2004 effectively expulsed MSX for the remainder of the study period. Thus both MSX and Dermo were at high prevalence in 1995 (no biomass estimate is available for this time) and 2002 (second lowest biomass on a decreasing trend that began in 1998). Both 1995 and 2002 were, notably, years of

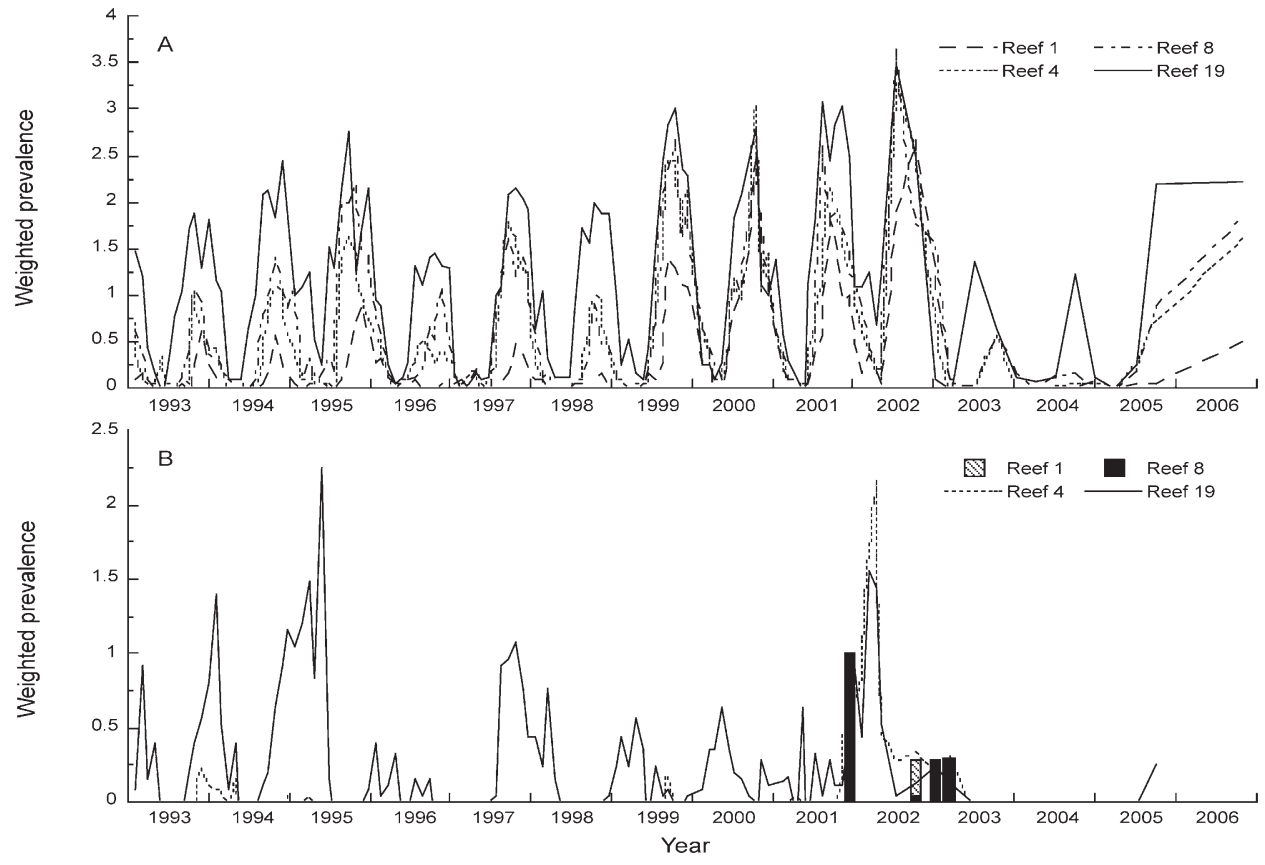

Figure 10. Weighted prevalence of Perkinsus marinus (A) and Haplosporidium nelsoni (B) at reefs 1, 4, 8, and 19 from 1993 to 2006. Haplosporidium nelsoni prevalence at reefs 1 and 8 were only collected from 2002 to 2006. Note the difference in scale between the two panels. Weighted prevalence is on a scale of 0 (no disease detected) to 5 (100\% with heavy infections). 
TABLE 10.

Estimation of 1999 y class serial contribution (L) to the shell habitat base for reef 6 . See text for additional details.

\begin{tabular}{|c|c|c|c|c|c|c|c|}
\hline \multirow[b]{2}{*}{ Age } & \multirow[b]{2}{*}{$n$} & \multirow[b]{2}{*}{ \% Mortality } & \multicolumn{2}{|c|}{$\begin{array}{c}\text { Shell Per } \\
\text { Individual } \\
\text { (L) }\end{array}$} & \multicolumn{2}{|c|}{$\begin{array}{c}\text { Shell } \\
\text { Total } \\
\text { (L) }\end{array}$} & \multirow{2}{*}{$\begin{array}{c}\text { Year Added } \\
\text { to Shell } \\
\text { Base }\end{array}$} \\
\hline & & & Low & High & Low & High & \\
\hline 0.33 & 166.8 & & & & & & \\
\hline 1.33 & 108.7 & 58.2 & 0.02 & 0.06 & 1.25 & 3.32 & 2000 \\
\hline 2.33 & 49.2 & 59.4 & 0.06 & 0.11 & 3.40 & 6.64 & 2001 \\
\hline 3.33 & 4.5 & 44.7 & 0.11 & 0.19 & 5.00 & 8.33 & 2002 \\
\hline 4.33 & 0.2 & 4.4 & 0.19 & 0.28 & 0.82 & 1.23 & 2003 \\
\hline
\end{tabular}

high recruitment resulting in the two highest standing stock levels (as indicated by oyster density) during the study period.

\section{Contribution of Mortality to the Shell Habitat Base}

Mortality contributes to the shell habitat base and the persistence of the essential habitat for oysters (Powell \& Klinck 2007, Mann \& Powell 2007). The challenge in estimating such contributions in the current data set is a lack of knowledge as to when in the year between successive surveys that an individual dies. Consider an example for an individual that dies in the transition from 2-3 y of age. The death may occur the day after the survey, dictating use of a length versus shell estimator for a 2-y-old. If the mortality occurs the day before the subsequent survey it would be more appropriate to use the length versus shell estimator for a 3-y-old. Thus Table 10 has both low and high estimates of individual and shell total (population) contribution to the shell base with mortality events on reef 6 (note this is the same high density reef outlined in Table 9). The end product is a tabulation of high and low estimates of serial contribution to the shell base by the 1999 year class in the years 2000 to 2003 associated with mortality of the cohort in those years. The shell totals in Table $10(1.25,3.40,5.00$, and 0.82 for low, 3.32, 6.64, 8.33, and 1.23 for high) are underscored values in Table 11 that assembles a larger, multiyear class data set as illustrated below.

The first and second sections of Table 11 allow estimation of serial addition of shell for each calendar year (1999 to 2006) for all of the contributing cohorts as both low and high estimates as described earlier and in Table $10.10 \mathrm{~L} \mathrm{~m}^{-2}$ is equivalent to a uniform layer $1 \mathrm{~cm}$ thick spread over $1 \mathrm{~m}^{2}$. There is a single negative value in these iterations, $1.33 \mathrm{y}$ in 2006. The value is modest compared with others in the described time frame, -1.0 and $-2.8 \mathrm{~L} \mathrm{~m}^{-2}$ respectively for low and high estimates. The third section of Table 11 is accounting and estimating: the line "observed" is the total shell $\mathrm{m}^{-2}$ recorded for the surveyed year. Thus the expected shell amounts, assuming no losses, for the subsequent year $(t+1)$ would be shell at time zero plus an amount somewhere between the low and high estimate produced by mortality - the lines labeled "observed $(\mathrm{t}-1)+$ low" and "observed $(\mathrm{t}-1)+$ high". It is obvious that these accumulations do not occur, the difference is the shell loss to degradation processes, and this is estimated in the rows labeled "loss (low)" and "loss (high)". Expressing these as either absolute $\left(\mathrm{L} \mathrm{m}^{-2}\right)$ or percentage rates illustrates the rapid turnover of the all important habitat that have, with the notable exception of the work of Powell and colleagues (Powell et al. 2006, Powell \& Klinck 2007), been marginally considered but not adequately quantified. To illustrate shell loss rates the survey data was assembled for all seven high and medium density reefs (Fig. 11A, reefs 3 through 9 and 23, see earlier text) and all remaining low-density reefs (Fig. $11 \mathrm{~B}$, reefs 1,2 , and 10

TABLE 11.

Estimation of serial addition of shell (L) from 1999 to 2006 on reef 6 from all of the contributing cohorts as both low and high estimates as described in Table 10. See text for additional details.

\begin{tabular}{|c|c|c|c|c|c|c|c|c|}
\hline Age & 1999 & 2000 & 2001 & 2002 & 2003 & 2004 & 2005 & 2006 \\
\hline 1.33 & 0.5 & $\underline{1.2}$ & 1.1 & 2.5 & 10.4 & 5.5 & 0.2 & $? 1.0$ \\
\hline 2.33 & 3.5 & $\overline{1.1}$ & $\underline{3.4}$ & 5.7 & 2.0 & 1.3 & 3.3 & 4.3 \\
\hline 3.33 & 5.6 & 2.7 & 3.0 & $\underline{5.0}$ & 1.5 & 0.4 & 1.9 & 5.9 \\
\hline 4.33 & 1.8 & 1.2 & 1.7 & $\overline{1.8}$ & $\underline{0.8}$ & 0.2 & 1.0 & 1.5 \\
\hline Low estimate total & 11.4 & 6.1 & 9.2 & 15.0 & 14.7 & 7.4 & 6.4 & 10.6 \\
\hline 1.33 & 1.3 & $\underline{3.3}$ & 3.0 & 6.6 & 27.9 & 14.6 & 0.4 & $? 2.8$ \\
\hline 2.33 & 6.8 & 2.1 & $\underline{6.6}$ & 11.1 & 4.0 & 2.6 & 6.5 & 8.3 \\
\hline 3.33 & 9.4 & 4.4 & 5.0 & 8.3 & 2.4 & 0.7 & 3.2 & 9.8 \\
\hline 4.33 & 2.7 & 1.7 & 2.6 & $\overline{2.8}$ & 1.2 & 0.3 & 1.6 & 2.3 \\
\hline High estimate total & 20.2 & 11.6 & 17.2 & 28.8 & $3 \overline{5.5}$ & 18.1 & 11.6 & 17.7 \\
\hline Observed & 24.5 & 27.6 & 30.0 & 32.9 & 28.9 & 22.9 & 26.0 & 28.0 \\
\hline Observed $(\mathrm{t}-1)+$ low & 34.1 & 30.6 & 36.8 & 45.0 & 47.6 & 36.3 & 29.3 & 36.6 \\
\hline Observed (t-1) + high & 43.1 & 36.1 & 44.8 & 58.8 & 68.4 & 47.0 & 34.5 & 43.7 \\
\hline Loss (low) & 9.6 & 3.0 & 6.8 & 12.1 & 18.7 & 13.4 & 3.3 & 8.6 \\
\hline Loss (high) & 18.6 & 8.5 & 14.8 & 25.9 & 39.5 & 24.1 & 8.5 & 15.7 \\
\hline Loss $(\%$, low $)$ & 28.2 & 9.9 & 18.5 & 26.9 & 39.3 & 36.8 & 11.3 & 23.5 \\
\hline Loss $(\%$, high $)$ & 43.1 & 23.6 & 33.0 & 44.0 & 57.7 & 51.3 & 24.7 & 35.9 \\
\hline Loss $(\%$, med $)$ & 35.7 & 16.8 & 25.8 & 35.4 & 48.5 & 44.1 & 18.0 & 29.7 \\
\hline
\end{tabular}



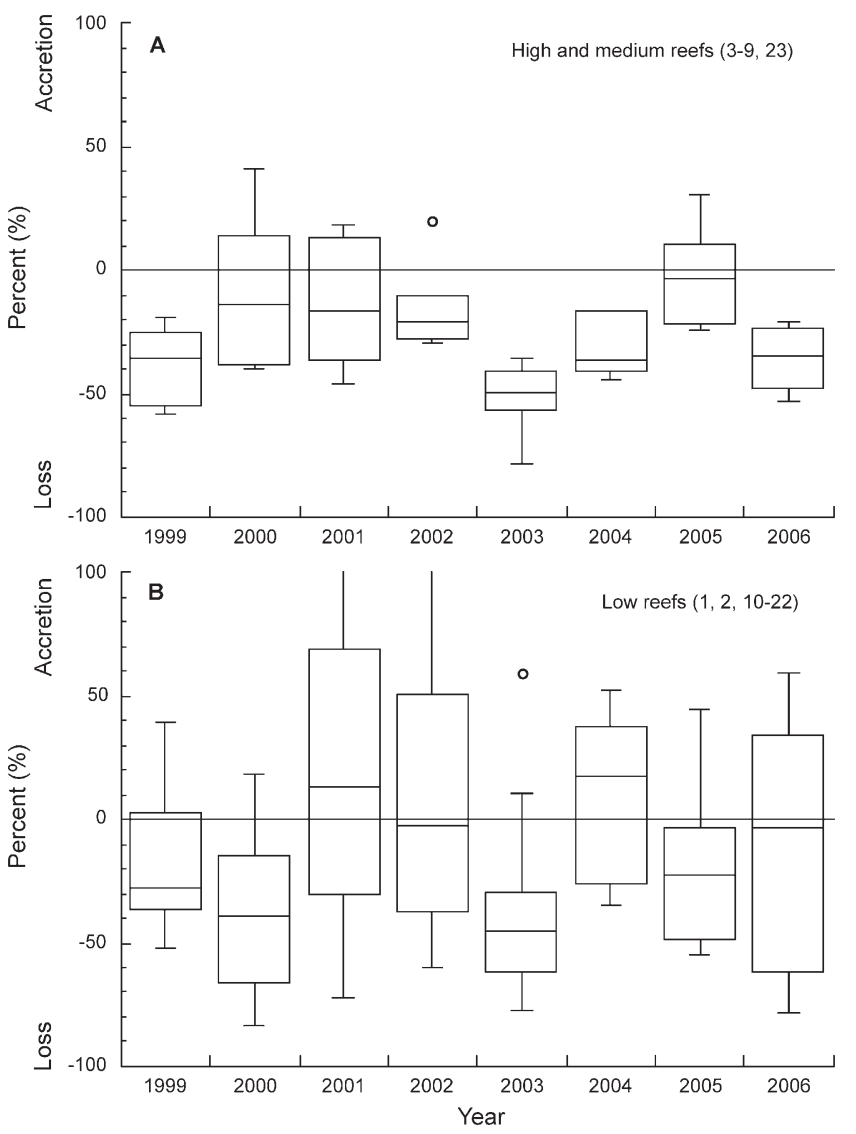

Figure 11. Shell loss rates for the 1999 to 2006 period for the high and medium density reefs (A) and low density reefs (B). Each point is represented as median 25 th and 75 th percentile, minimum and maximum value. See text for additional details.

through 22). Each value is assembled first using the means of the low and high loss rates for each reef for the years 1999 to 2006, and then expressing a single annual value as the mean, standard deviation and range for the assembled means. Values below zero represent percentage loss of shell per year and are negative. Conversely, values above zero represent shell accretion and have positive values. Figure 11 data have not been filtered. Figure 11A (high and medium reefs) contains six positive values (mean of high and low rate loss estimations) in the high and medium density reefs. Four of these are in the range 0 to minus $10 \%$ and are probably within the range of cumulative error from this sequential calculation. The remaining two values from the 56 represented in the plot, suggest shell accumulation rather than loss, one in 2000 and one in 2005 . Both are related to one atypically low observed shell value in the time sequence and may be a product of field sampling error in that the Bros and Cowell (1987) delimiter was based on live numbers and not shell in the field. The majority of the values are at or above $20 \%$ shell loss rates per year, many in the $30-50 \%$ range! Considering the loss rate function for high and medium density reefs as a halflife rate plot of the remaining proportion of the original shell base versus years suggests rate losses for the described reefs with half lives in the $\leq 3$-y-range. These are consistent with recent estimates by Powell and collaborators (Powell et al. 2006, Powell \& Klinck 2007) for Delaware Bay. The scatter in values for the low-density reefs (Fig. 11B) is considerable. Whereas most values again illustrate shell loss several values are in excess of $50 \%$ accretion estimates. These are, however, locations with standing shell estimates generally $<10 \mathrm{~L} \mathrm{~m}^{-2}$ (Table 3 ). At such low densities, error in estimation is large considering the possibility of aggregation of individual shells within the one $\mathrm{m}^{2}$ sampling unit (see earlier discussion of variance to mean ratios for shell volume values and the patchy distribution of the shell resource).

\section{Oyster Shell as Habitat}

The proportion of brown shell to total shell trends upwards with increasing total shell volume (Fig. 5), indicating a refractory, buried base component, the black shell, with a more variable, overlaying brown shell (the brown probably has a shorter half life). A notable increase in total shell volume occurs at and above brown shell proportions of 0.5 . Proportional values $\leq 0.4$ correspond to average total shell volumes of $\leq 7-$ $10 \mathrm{~L} \mathrm{~m}^{-2}$. Supplemental shell addition, described in Table 8, occurred in 1994 through 1996, and in 1998 as part of a fishery replenishment program. These were targeted additions, modest in terms of total shell present but locally increasing density.

Figure 12A presents a plot of total shell (brown, black, and live) versus live shell weight. The plot appears to have two phases separating at an average total shell volume of approximately $2 \mathrm{~kg} \mathrm{~m}^{-2}$ or $3.4 \mathrm{~L} . \mathrm{m}^{-2}$, note the correspondence with Figure 5. We suggest that the $2 \mathrm{~kg} \mathrm{~m}^{-2}$ or $3.4 \mathrm{~L} . \mathrm{m}^{-2}$ value represents buried, black shell that is unavailable for recruitment. In Figure $12 \mathrm{~B}$ data points corresponding to total shell values of $<2 \mathrm{~kg} \mathrm{~m}^{-2}$ are removed and the remaining 166 data pairs replotted with the addition of median values on each axis. Thus data are divided into 4 quartiles corresponding to low shell + low live $(n=76)$, low shell + high live $(n=6)$, high shell + high live $(n=76)$, and high shell + low live $(n=8)$. Of particular note is the distribution of the resultant data, with $92 \%$ of the points being equally distributed between the low shell + low live, and the high shell + high live quartiles. The other quartiles are poorly represented and are suggested as unstable conditions. The quartile of low shell + high live would only result from a high recruitment event on limited substrate followed by high survival. We suggest that this scenario is unlikely and atypical. The high shell + low live quartile represents a postepizootic mortality event of adults. This is a transient condition in that the shell component degrades in the absence of continued input through mortality, with the eventual transition of the location to a low shell + low live condition. The distribution of the data points in Figure 12B suggest regions of poor (low shell + low live) and good (high shell + high live) habitat with associated live oyster populations. The data within the high shell + high live quartile present an approximate 1:3 relationship between live shell and total shell, or 1:2 between live shell and non living available substrate, effectively brown shell given that the black shell has been estimated at $2 \mathrm{~kg} \mathrm{~m}^{-2}$ and removed from the calculation. Much lower proportions of live shell to total shell are expected in low quality habitat as illustrated by the points in the low shell + low live quartile in Figure 12B. To further examine this relationship, data from Figure 12B are replotted in Figure 12C as the annual value for the proportion of average live shell to total shell by weight for the period 1998-2006. Each year is represented as median 25 th and 75 th percentile, minimum and maximum 

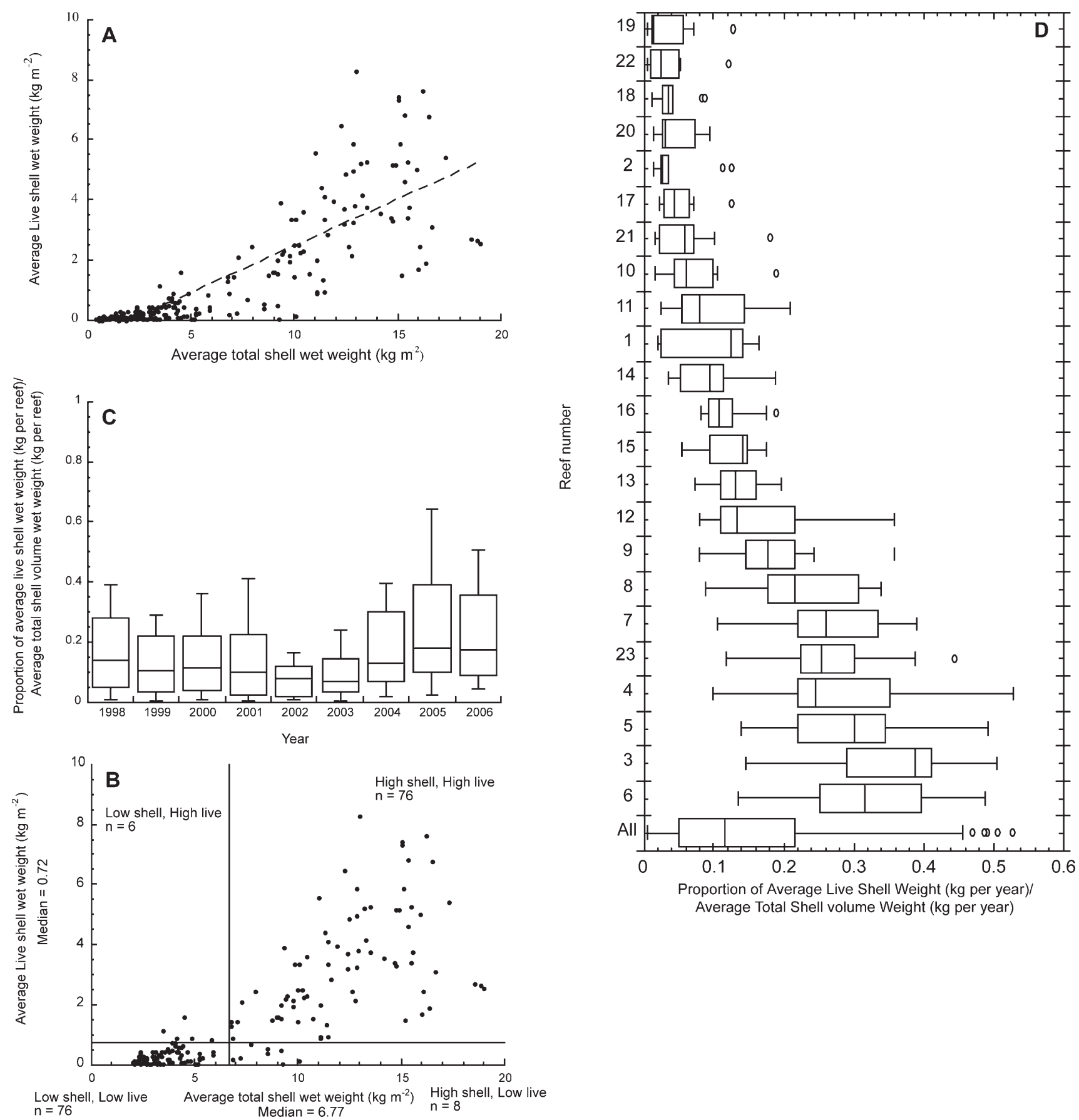

Figure 12. The relationship of live oyster shell wet weight $(\mathrm{kg})$ to total shell wet weight $(\mathrm{kg})$ on a per unit area basis, for all reefs, all years (A); all reef year combinations with average total shell wet weight $>2 \mathrm{~kg} \mathrm{~m}^{-2}(\mathrm{~B})$. The proportion of average live shell wet weight to total shell wet weight by year (C) and by reef (D) for the period 1998 to 2006. For panel B, median values are included on both axes. For panel C and D, each year (panel C) and reef (panel D) is represented as the median, 25th and 75th percentile with minimum and maximum values.

values for 23 reefs per year. All years have minimum values $<<0.05$ (essentially all shell with no live oysters). The 25 th percentile value varies between 0.05 and 0.1 . Median values vary between 0.075 and 0.175 . The maximum value of the upper quartile varied by a factor of 2.6. The 75th percentile and maximum values are notably lower in 2002 and 2003 than the remaining years at 0.25 . The year 2003 was a disease epizootic, high mortality year, and whereas recruitment was high in 2002 , the contribution of the new recruits to the live shell estimate was minimal. Higher standing stock years of 2004 through 2006 are represented by higher proportion values consistent with freshets and low disease pressure in 2004 to 2005. Elevated disease pressure returned in 2006 and the upper quartile was lower than its 2005 value. In addition the distribution of the 75th and maximum values above the median indicate a modest number of reefs with markedly better habitat and live populations than 
the surrounding majority of the reefs. This is consistent with the general patterns of the live population distribution described by Tables 1 and 2. Replotting the data of Figure 12C by reef rather than year, as shown in Figure 12D, underscores the conclusion that reefs in the system group as high quality habitat with live populations (reefs 3-9 inclusive plus 23) with consistently higher median values than the low quality habitat (reefs 2, 10-22 inclusive) with poor populations with no intermediate populations.

The portrayal of the live shell to total shell relationship as quartiles representing various habitat qualities prompts the question as to the possibility of defined habitat moving from one quartile to another over a period of time. Does habitat recover to a higher quality within the time period of the study? Does the stability or instability of a habitat within a quartile suggest that habitat can be manipulated to provide a stable, high quality status consistent with restoration and rebuilding goals? To examine these questions the quartile plot illustrated in Figure 12B was replotted as 23 individual reef plots. Eight of these are presented as Figures 13A to H, with each plot describing one reef for the 1998 to 2006 period. The time sequence of points for each reef was then examined for stability within one quartile. The results of this analysis are remarkable. Eleven reefs (low quality habitat) were confined for the entire period to the low shell + low live quartile (reefs 1, 2, 13-15 inclusive, and 17-22 inclusive). Seven reefs (reefs 3-9 inclusive) were confined to the high shell + high live quartile whereas an eighth reef (reef 23) maintained eight of nine years comfortably within the high shell + high live quartile with a single data point (2003) in the previously described unstable quartile of low shell + high live. This single 2003 data point illustrates a rapid and modest transition into and out of this quartile accompanying a high mortality, high recruit year. However, all other data points in this eight reef collective, the high quality habitat, are resilient to even epizootic associated high mortality events over the study period. The distribution of the time sequence of data points in these eight reefs within the high shell + high live quartile is remarkably consistent. The 1998 to 1999 transition is generally in a lateral or predominantly downward direction indicating lower absolute live shell values. The 1999 to 2003 transitions result in comparable live shell values in seven of the eight reefs, the notable exception being reef 23 with a single data point in the low shell + high live quartile in 2003. The high recruitment event in 2002 resulted in a uniform and sequential increase in the live shell value in both 2004 and 2005. Increasing mortality in 2006 resulted in a decrease in the live shell value in all reefs except reef 6 for that year.

Of the remaining four reefs (reefs 10-12 inclusive and 16) that did not fall within either the high or low quality habitat, one demonstrated a single, marginal transition from low shell + low live to low shell + high live in 2005; a second (reef 12) demonstrated a transition from low shell + high live to low shell + low live in 1999, with a reverse of this transition in 2004; and a third reef (reef 10) oscillated between low shell + low live and high shell + low live with a single data point (2006) in the high shell + high live quartile. In only one instance (reef 11), were there observations of a transition from the low shell + low live and high shell + low live to the high shell + high live quartile for the 2004 to 2006 period. Whereas this trio of data points is within the good habitat quartile they are low in absolute shell and live shell densities when compared with stable reefs (such as reefs 3-9 and 23) within that quartile.

A final plot was made (Fig. 14) to examine the relationship between recruitment and live shell habitat. This is a stock (year t) versus recruit (year $\mathrm{t}+1$ ) plot that includes all data on a reef specific basis. Unlike the system wide stock versus recruit curve in Figure 7 that assumes connectivity within the reef system but does not assign recruits to specific source reefs, Figure 14 describes only the relationship between recruitment and the presence of live adult ( $>1$ y old) oysters. The strong linear relationships (Table 12) described by Figure 14 underscore that arguably more than anything else, the presence of live oysters supercedes all other factors in both encouraging recruitment and perpetuating populations. Whereas the live substraterecruit relationships within years are reasonably robust, the variation observed between years is large (Fig. 14B) and may be the product of environmental, disease, or demographic events related to both. The slopes of the yearly regressions are the annual replacement ratios. With three exceptions ( $>1$ : 2002, 2003, and <1: 2006), the replacement ratios for the James River oyster population are on the order of one.

\section{DISCUSSION}

There are few literature descriptions of absolute densities of oysters on natural reefs over historical time frames. The paucity of such published data sets is a product of the use of dredges for semiquantitative sampling in fishery support mode. Such approaches are limited without extensive calibration (e.g., Powell et al. 2002) that are typically lacking (see comments in Mann et al. 2004). In the upper James River, high and medium density reefs (reefs $3-9$ and 23 inclusive) occupy only $5.4 \%$ of total area, yet they contain between 25.7 and $55.5 \%$ by number and $35.8 \%$ to $54.8 \%$ by biomass of the oyster population. Understanding the mechanisms that maintain these concentrated areas over time is important. Reefs 3-7 occur within the area of the river where both the surface and bottom currents make a major turn from upriver to downriver (Fig. 1, Ruzecki \& Hargis 1989). This is the only area in the system where there is convergence between the bottom and surface layers. Reefs 8, 9 and 23, which fall out between reefs 3-7 and the rest of the system in terms of both oyster density and biomass, occur slightly downriver of this convergence zone (Fig. 1)

Above average recruitment, as noted by increases in reef specific density (1998, 1999, 2002, 2004, and 2006; Table 1) is typically observed after winter temperatures remain at or above $8^{\circ} \mathrm{C}$. Mann and Evans (2004) describe $8^{\circ} \mathrm{C}$ as a minimum temperature for growth in the James River system. Wet winters with salinity values $<9$ ppt (Fig. 3) reinforce the recruitment and survival signal in the following summer. High salinity in the following summer also reinforces the relationship with winter temperature in that high salinities were observed in all the years with above average recruitment except 2004. In 2004 recruitment was higher in the more saline, downstream region of the river. Elevated summer salinity also facilitates disease epizootics with resultant mortality as discussed elsewhere in this text.

\section{Stock Recruit Curve and Replacement Ratios}

Despite annual replacement ratios approximating or exceeding 1 in 13 out of the 14 y examined, the impacts of very high 
Oyster Population Biology
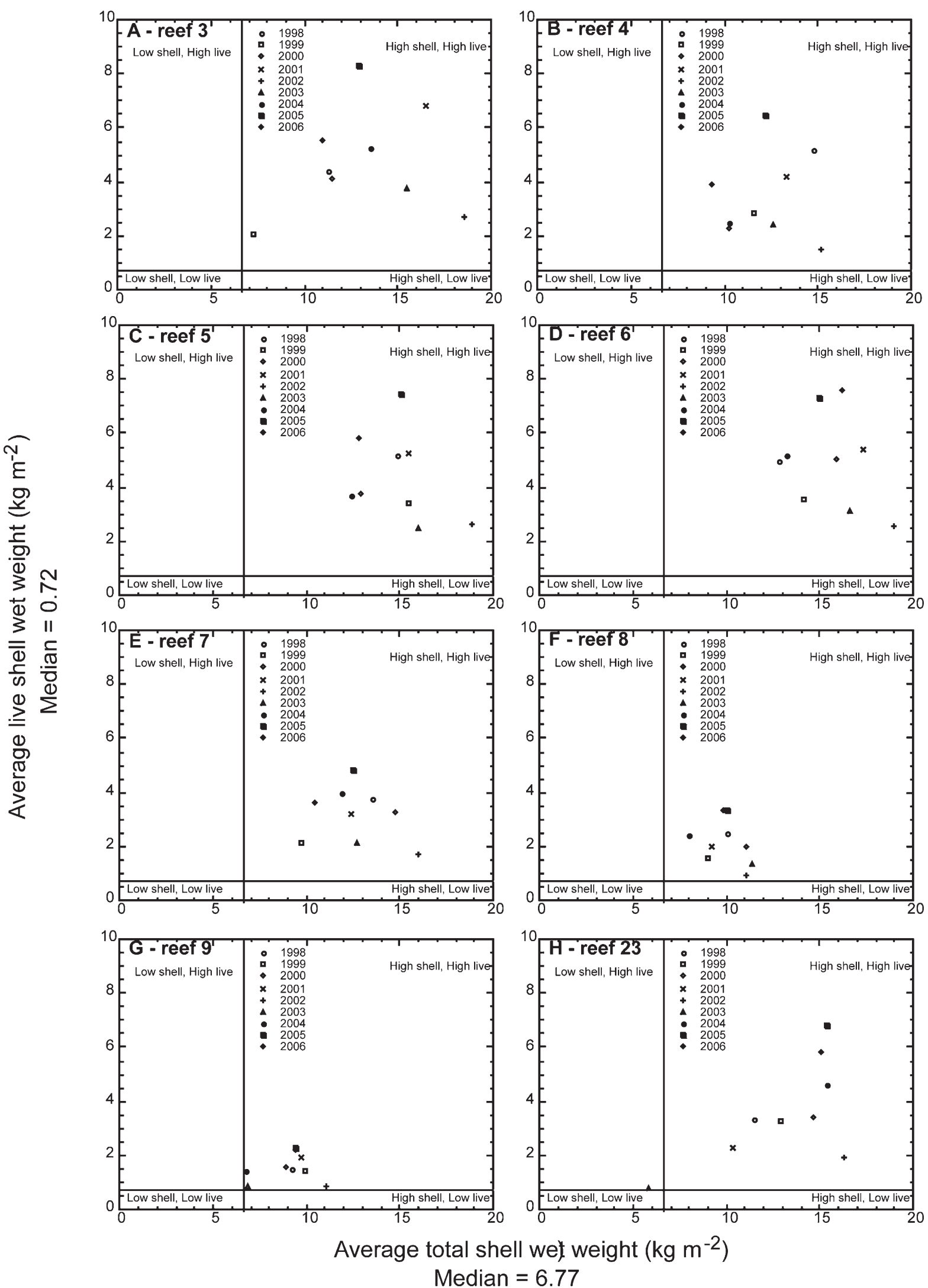

Figure 13. A to $\mathrm{H}$. Quartile plots of average live shell wet weight $\left(\mathrm{kg} \mathrm{m}^{-2}\right)$ versus average total shell wet weight $\left(\mathrm{kg} \mathrm{m}^{-2}\right)$, with medians from Figure $12 \mathrm{~B}$ plotted on both axes for eight individual reefs for the period 1998 to 2006. 

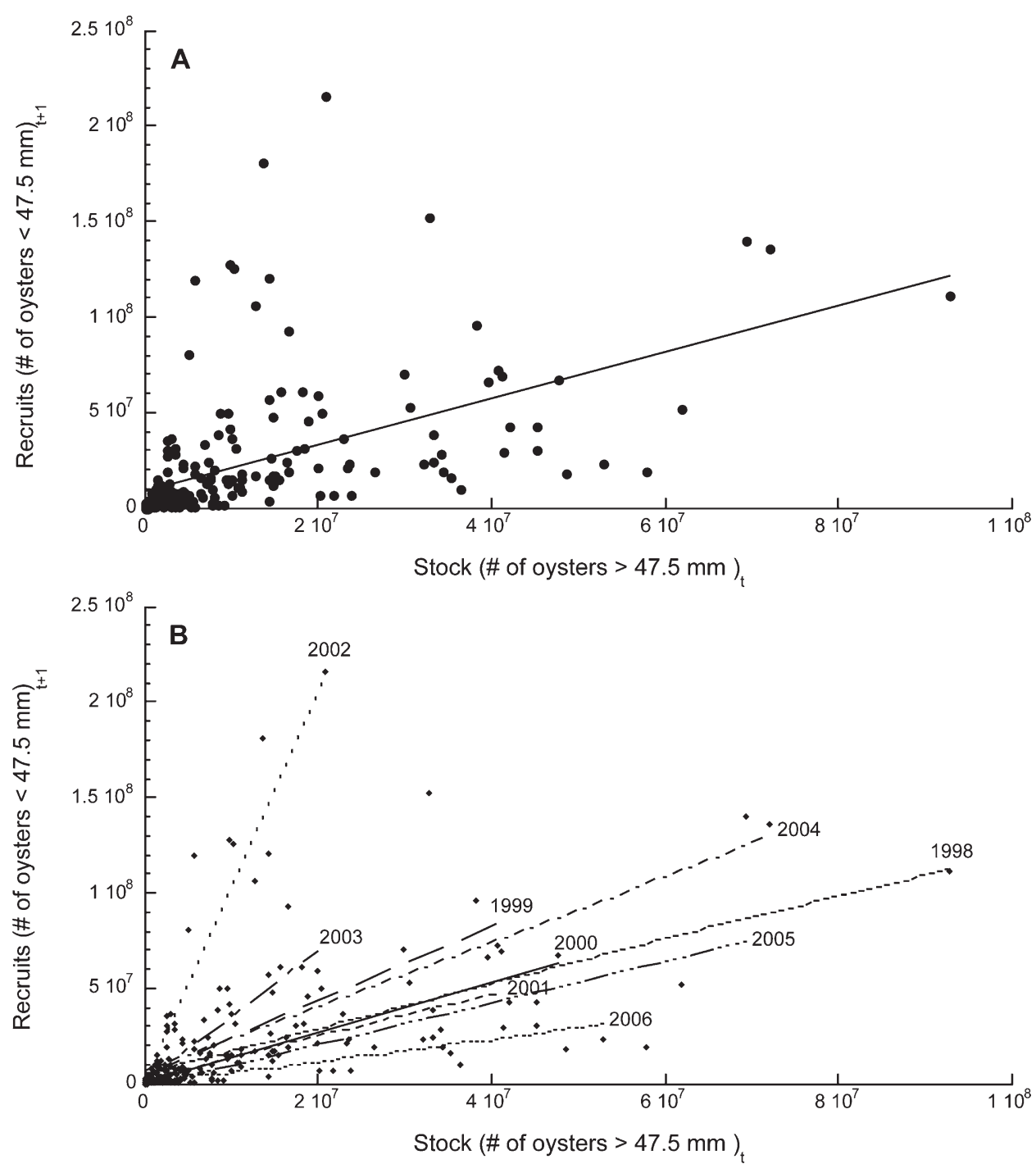

Figure 14. Standing stock (year $t$, oysters $\mathrm{m}^{-2}$ ) versus recruits $\left(\right.$ year $\mathrm{t}+1$, oysters $\mathrm{m}^{-2}$ ) on a reef specific basis, all years and reefs (A); all years and reefs with year specific lines fitted (B).

proportional mortality rates at all ages result in a failure to build the population over the course of the study. A combined consideration of replacement ratios and life expectancies prompts speculation as to the time frame to rebuild a population in the event of reduced frequency or intensity of epizootic events and associated reductions in mortality. A sustained generational replacement ratio of 1.5 in combination with a life expectancy of $6 \mathrm{y}$ results in a slow but exponential increase in total numbers. In 5 generations, that is $30 \mathrm{y}$, the possibility of the total population growth in number is by $1.5 \mathrm{x}, 2.25 \mathrm{x}, 3.4 \mathrm{x}$, $5.1 \mathrm{x}$, and $7.6 \mathrm{x}$ with each contributing generation. A life expectancy of $10 \mathrm{y}$ gives a $2.5 \mathrm{x}$ replacement with each generation and a cumulative increase in number of 15.6x. These simple calculations do not include the added value of both larger size and protandric hermaphroditism in oysters. If the number of female oysters increases in the larger length classes, egg production per individual will increase (both because the females are larger and the assumption that the proportion of females increases). With time, there would be the expectation for the stock to recruit relationship, currently more or less absent, to change assuming habitat is not limiting. The live stimulus effect illustrated in Figure 14 will also positively affect the stock to recruit relationship.

Even a modest sustained trend of recruitment and increased life expectancy begs the question of how long would be required to rebuild the stock to carrying capacity in the scenarios of reduced or removed epizootics? The question of habitat being limiting is bounded by the calculation of the very limited proportion of the substrate required by a typical recruiting year class, much less than $1 \%$, versus the limited half-life of the dead shell as a recruitment substrate. The increased longevity of individuals prior to death is paramount here in assuring that a single year class contributes to the shell base over many years, thus mitigating the impacts of irregular recruitment. Even in the presence of increased individual longevity, the occlusion of substrate and/or decay of that substrate with sequential years of poor replacement also bodes poorly for perpetuation of a population. Unfortunately, the current observations illustrate a period wherein high disease pressure typically co-occurs with higher recruitment, and then higher mortality prevails in the 
TABLE 12.

Statistics for stock recruit relationships (1998-2006) plotted in Figure 14.

\begin{tabular}{lcccccc}
\hline \hline & & & & \multicolumn{3}{c}{ n Data } \\
\multicolumn{1}{c}{ Year } & Slope & SE Slope & Y Intercept & SE Intercept & Pairs & R2 \\
\hline 1998 & 1.1524 & 0.1719 & $5.84 \times 10^{6}$ & $4.74 \times 10^{6}$ & 23 & 0.68 \\
1999 & 1.9582 & 0.3321 & $4.15 \times 10^{6}$ & $4.27 \times 10^{6}$ & 23 & 0.62 \\
2000 & 1.3136 & 0.1107 & $5.66 \times 10^{5}$ & $1.74 \times 10^{6}$ & 23 & 0.87 \\
2001 & 1.1205 & 0.1267 & $1.91 \times 10^{6}$ & $1.94 \times 10^{6}$ & 23 & 0.79 \\
2002 & 10.1852 & 0.9039 & $1.02 \times 10^{6}$ & $6.84 \times 10^{6}$ & 23 & 0.86 \\
2003 & 3.3887 & 0.2087 & $1.90 \times 10^{6}$ & $1.58 \times 10^{6}$ & 23 & 0.93 \\
2004 & 1.7038 & 0.3954 & $6.73 \times 10^{6}$ & $8.70 \times 10^{6}$ & 23 & 0.47 \\
2005 & 1.0954 & 0.2223 & $1.92 \times 10^{6}$ & $6.20 \times 10^{6}$ & 23 & 0.54 \\
2006 & 0.5544 & 0.0677 & $1.66 \times 10^{6}$ & $1.27 \times 10^{6}$ & 23 & 0.76 \\
All years & $\mathbf{1 . 2 1 4 4}$ & $\mathbf{0 . 1 3 3 8}$ & $\mathbf{8 . 9 0} \times \mathbf{1 0}^{\mathbf{6}}$ & $\mathbf{2 . 5 0} \times \mathbf{1 0}^{\mathbf{6}}$ & $\mathbf{2 0 7}$ & $\mathbf{0 . 2 9}$ \\
\hline
\end{tabular}

spawning adult stock. Only relief from the epizootics appears to have the potential to increase and maintain the spawning stock size and foster a period of population rebuilding.

\section{Disease impacts on mortality}

Observations of Dermo incidence and prevalence are in accordance with previous observations of Ford and Tripp (1996) for Delaware Bay. The relative importance of Dermo as a causative agent of mortality appears greater than that of MSX. The regular incursion of one or both diseases in high salinity years offers the opportunity for selection of tolerant individuals. The massive epizootic of MSX in Delaware Bay in 1985-1986, followed by the practical disappearance of that disease in subsequent years (HSRL 2007, Mann \& Powell 2007) suggest that selection for tolerance to MSX is possible in short time periods under fortuitous conditions. Unfortunately, the opportunity for such an event in the James River is limited by geography in that the upper river is a low salinity refuge, which rarely experiences massive epizootic related mortalities even in low river flow years. Large numbers of effectively unselected larvae can be contributed to the system wide recruit pool each year. Additionally, exploitation of selected survivors occurs in the higher salinity downstream regions. That is, the 3 plus year olds that remain after high cumulative mortality (Fig. 8) are the prime targets for commercial fishing. Consider a 0.3 proportional survival for each of three successive years for a cohort that recruits at 100 oysters $\mathrm{m}^{-2}$. The result is less than 3 oysters $\mathrm{m}^{-2}$ of surviving broodstock with these larger oysters in downstream locations representing a small percentage of the standing stock by number.

\section{Shell Loss Rates}

Shell loss continues unabated independent of any changes in recruitment and mortality. High recruitment in combination with high mortalities simply results in rapid turnover of a modest shell reservoir. Thus a situation wherein both mean values of change in oyster numbers $(\mathrm{dN} / \mathrm{dt})$ and change in shell $(\mathrm{dS} / \mathrm{dt}) \approx 0$ is maintained over extended periods, can still reflect a limited shell resource. To accrete (build) reef structures in excess of shell loss, there is a requirement for both sustained recruitment and, especially, survival to greater age before individuals die. The absence of both of these requirements in a restoration scenario leads to net shell substrate loss, eventual recruitment failure and loss of the population. Given the current disease situation in extant populations, a change to a consistent accretion situation is unlikely to occur.

The long-term $\mathrm{dN} / \mathrm{dt}$ and $\mathrm{dS} / \mathrm{dt} \approx 0$ double reference point sets the minimal limit for a capture fishery. Powell and collaborators have completed such an analysis for Delaware Bay (HSRL 2007). There is no such analysis published for any oyster fishery within the Chesapeake Bay, and this is clearly overdue. The proposed use of alternate substrates to prevent a decline in shell substrate (consistent negative $\mathrm{dS} / \mathrm{dT}$ trends) does little to help. Assuming that such installations are supposed to act as a metapopulation source and provide a $\mathrm{dS} / \mathrm{dt}$ that in theory should not change, they do nothing for sites (sinks) to which they are argued to export larvae. If the recipient sites cannot become at least shell neutral the habitat disappears in a time course commensurate with Figure 11 so the source is of no practical application.

The quartile analyses of Figures 12 and 13 suggest that stable states exist with respect to both population density and shell habitat. Simple creation of high density reef structures with addition of broodstock, the obvious desired end points of rebuilding as either sources or sinks, cannot come with expectations of being self-sustaining unless a complete population demographic is accompanied by a drastic reduction in epizootic frequency and intensity. A recent analysis by Southworth et al. (2008) suggests that the desired consistent and very high recruitment is not observed in the improved habitat locations. Indeed, an examination of data from 243 -dimensional reefs and shell plants, in the Virginia portion of the Chesapeake Bay and its sub estuaries typically exhibit a year or two of recruitment before the signal decays. Restoration is arguably untenable without a self-sustaining system in the absence of continuing manipulation. Even the most optimistic projections from this analysis suggest that multiple, sequential manipulative actions will be required to provide an end point with a self-sustaining population. We predict the associated end point cost estimate will be frightening and far from cost neutral if the intent is to provide the $\mathrm{dN} / \mathrm{dt}$ and $\mathrm{dS} / \mathrm{dt} \approx 0$ scenario for any capture fishery in the Chesapeake Bay.

\section{Live oyster: oyster shell relationship}

Recall that the dominating factor in adult density is not the stock - recruit relationship, but the survival per recruit (replacement ratio). A strong relationship is observed between available (brown shell, above the sediment- water interface) substrate and live shell. The latter is a function of recruitment and subsequent mortality. Thus a strong relationship between available substrate and recruitment is supported by all plots in Figures 12, 13, and 14 as well as population density values in Table 1 . The density data also underscore how little of the total area of the substrate is occupied by new recruits. Again, consider an example. A substrate value of $10 \mathrm{~L} \mathrm{~m}^{-2}$ equates to $5.8 \mathrm{~kg} \mathrm{~m}^{-2}$ and covers one square meter to a uniform depth of $1 \mathrm{~cm}$. Assume this to be $1 \mathrm{~m}^{2}$ of potentially available substrate. Five hundred recently recruited spat, each of approximately $1 \mathrm{~mm}^{2}$ area, would occupy only $0.02 \%$ of that substrate. The apparent surplus of brown shell surface indicates that fouling or competing organisms occupy the vast majority of the shell surface. This is in accordance with Rheinhardt and Mann (1990), and underscores the importance 
of strategic timing of shell planting in replenishment and restoration efforts. The high proportion of live shell to total shell in high quality habitat suggests that available, that is, unfouled substrate, rather than total substrate is limiting. Oyster growth edges provide a continual supply of new, clean substrate. Youngof-the-year oysters should recruit on actively growing oysters in undisturbed systems where long-lived oysters prevail and where recruitment, growth and mortality contribute to reef accretion. These dynamics are rarely recorded in extant subtidal systems, where accretion is minimal, and these relationships are inadequately appreciated in current restoration efforts.

The extensive data set on the relationship of live shell to total shell (habitat) illustrates that, despite variations in population density and biomass over the entire time course of the study, the probability of a single reef moving from one of the described quartiles to another is very small. The long-term data suggest that a low shell + low live quartile cannot be upgraded, and our cumulative knowledge of larval dispersal in the James River argues that the spatial biomass and habitat differences are not driven by larval supply differences. The causative factors of observed spatial population structure remain poorly understood, yet such understanding is central to management of the oyster resource. The implications of these observations on resource restoration and management are profound. Transition of any reef from a low shell + low live quartile to any other status is habitat rather than recruit limited. An insufficient amount of live shell is currently available from extant James River populations to increase the habitat available for recruitment in the short term.

Insufficient recruitment, demonstrated by the lack of live shell, dictates a long-term unavailability of shell to replace that lost to degradation. Transition of reefs from the low shell + high live quartile to other states requires mortality to provide habitat for further recruitment. Consistent recruitment has not recently occurred in the study area as demonstrated by the low total shell presence. Transition from the high shell + low live quartile requires consistent recruitment and survival to large size before mortality occurs. There are very few reefs in this category (eight points in Figure 12B). Even reefs in the high shell + high live quartile can be considered vulnerable. To maintain this status, a base total shell value of approximately $7 \mathrm{~kg} \mathrm{~m}^{-2}$, that is approximately $5 \mathrm{~kg} \mathrm{~m}^{-2}$ of brown shell (or $8.75 \mathrm{~L} \mathrm{~m}^{-2}$ or an equivalent uniform layer of approximately $9 \mathrm{~mm}$ ) with the remaining $2 \mathrm{~kg} \mathrm{~m}^{-2}$ of live shell, must be maintained. In the context of the above calculations, it is notable that in Virginia maintenance shell application to productive, commercially exploited reefs is approximately 1000 bushels per acre or $12 \mathrm{~L}$ $\mathrm{m}^{-2}$. Replenishment action is typically considered when total shell volume falls below $5 \mathrm{~L} \mathrm{~m}^{-2}$ or $2.9 \mathrm{~kg} \mathrm{~m}^{-2}$.

Estimates of the half-life of the shell base (sensu Powell et al. 2006) allow estimation of the size or age specific mortality rate to maintain this base. In turn this allows prediction of the sustained biomass of oysters that is required to maintain a balanced shell budget in periods of both biomass increase and in periods of high epizootic effects. Median shell loss rates for the high and medium density reefs vary over the 1999-2006 period depending on the balance between recruitment and mortality (Fig. 11) but even annual loss rates in the $20-30 \%$ range give half- lives in the 3-2 y range respectively.

The quartile based description of live oyster and shell dynamics described above may be used as a broad scale spatial descriptor of possible sources and sinks. Note that high shell + high live reefs can be located directly adjacent to low shell + low live reefs. These data suggest that shell and/or broodstock cannot be added to a habitat with the expectation of a stable high shell + high live reef as a result. It appears that the low shell + low live and the high shell + high live states have specific and extraordinarily stable features in their dynamics. Neither disease nor recruitment will overcome the threshold required to move these end point reefs toward a middle or transition state.

Consider that the reefs currently in the low shell + low live category may have at one time been very productive, possibly high shell + high live. This is particularly likely with regard to the downstream reefs (e.g., reefs 17-19, 21, 22). If this is the case, we have seen a stable point shift over time with regard to disease epizootics and over-fishing as well as other challenges. This transition from a previously stable high shell + high live state is consistent with the multiple stable point hypothesis proffered for Delaware Bay by Powell et al. (HSRL 2008, in review) wherein the production-biomass curve has two maxima with a minima in between rather than one maxima as in a classic Ricker curve. As epizootics caused the contraction of the occupied range of oyster habitat in Delaware Bay in recent years, the spatial footprint of the resource changed, as did the carrying capacity, thus resetting the upper end of the curve to a downward trend. The same thing happened in the James River however; quantitative stock assessment data predisease (circa 1950s) is not available. We suggest that the current observations are of productive pre-epizootic reefs being set to low shell + low live status in post epizootic conditions.

\section{Where do we go from here?}

Successful maintenance of self-sustaining oyster populations with concurrent positive reef accretion depends on achieving at least a 1:1 replacement ratio of recruits to broodstock over the long term. The very high mortality rates that we observe in the year 0 to year 1 transition combined with the consistently high mortality rates we see between all other year classes do little to meet this objective. Recruits are constantly exposed to high predation whereas all other ages are exposed to disease epizootics. Multiple consecutive years with replacement ratios of 5-6 recruits per broodstock are required to overcome this mortality. Existing replacement ratios of 1-2 are insufficient. Even if recruitment rates doubled, in the absence of a decline in the mortality rates the turnover rate of the substrate remains the same. Recruitment is not limiting in the James River. Mortality is the major problem. If mortality for all age classes can be reduced, the stock should gradually rebuild over the course of multiple generations. Successful rebuilding requires survival of more individuals to larger size classes (Age 6+, consider the oyster has a 10-20 y lifespan, Powell \& Cummings 1985), to contribute not only reproductive capacity (fecundity increases with size, Cox \& Mann 1992) but habitat/shell surface area as well. The prospect for this occurrence is bleak.

\section{ACKNOWLEDGMENTS}

This study was supported in part by funds from the NOAA Chesapeake Bay Stock Assessment Program (contract numbers NA66FU0487, NA07FU0539 and NA17FU2888), the NOAA Office of Sea Grant (NA56RGO141), and the Environmental Protection Agency (contract number CB983649-01-0), and throughout its entirety by funds from 
the Commonwealth of Virginia to VIMS and VMRC. Fieldwork assistance by many graduate assistants from VIMS and VMRC staff is gratefully acknowledged. We thank Dr. Eric Powell of Rutgers University for many constructive discussions. This contribution is dedicated to our VIMS colleagues whose work on oyster population biology in the James River provided the foundation on which we continue to build. They are Dexter
Haven, Emeritus Professor, and recipient of the National Shellfisheries Association Wallace Award, for his life long contributions in support of the oyster resources of Virginia; the late William J, Hargis Jr. Emeritus Professor and former Director of VIMS; Reinaldo Morales-Alamo; Kenneth Walker; Frank Walker; and the late James P. Whitcomb. This is Contribution Number 2989 from the Virginia Institute of Marine Science.

\section{LITERATURE CITED}

Andrews, J. D. 1984. Epizootiology of diseases of oysters (Crassostrea virginica), and parasites of associated organisms in eastern North America. Helgol. Meersunters. 37:149-166.

Andrews, J. D. 1988. Epizootiology of the disease caused by the oyster pathogen Perkinsus marinus and its effects on the oyster industry. Amer. Fish. Soc. Spec. Publ. 18:47-63.

Bartol, I. K. \& R. Mann. 1997. Small-scale settlement patterns of the oyster Crassostrea virginica on a constructed intertidal reef. Bull. Mar. Sci. 61:881-897.

Bartol, I. K. \& R. Mann. 1999a. Small-scale patterns of recruitment on a constructed intertidal reef: the role of spatial refugia. In: M. W. Luckenbach, R. Mann \& J. A. Wesson, editors. Oyster Reef Habitat Restoration: A Synopsis of Approaches. Virginia Institute of Marine Science, Gloucester Point, VA 23062. pp. 159-170.

Bartol, I. K. \& R. Mann. 1999b. Growth and mortality of oysters (Crassostrea virginica) on constructed intertidal reefs: effects of tidal height and substrate level. J. Exp. Mar. Biol. Ecol. 237:157184.

von Bertalanffy, L. 1938. A quantitative theory of organic growth (Inquiries on growth laws. II). Hum. Biol. 10:181-213.

Baylor, J.B. 1896. Method of defining and locating natural oyster beds, rocks and shoals. Oyster Records (pamphlets, one for each Tidewater, Virginia county, that listed the precise boundaries of the Baylor Survey). Board of Fisheries of Virginia

Benaka, L. R. 1999. Fish habitat: essential fish habitat and rehabilitation. American Fisheries Society, Bethesda, Maryland. 477 pp.

Bhattacharya, C. G. 1967. A simple method of resolution of a distribution into Gaussian components. Biometrics 23:115135.

Brooks, W. K. 1891. The oyster. Baltimore, Maryland: Johns Hopkins University Press. 230 pp.

Bros, W. E. \& B. C. Cowell. 1987. A technique for optimizing sample size (replication). J. Exp. Mar. Biol. Ecol. 114:63-71.

Burreson, E. M., M. E. Robinson \& A. Villalba. 1988. A comparison of paraffin histology and hemolymph analysis for the diagnosis of Haplosporidium nelsoni (MSX) in Crassostrea virginica (Gmelin). J. Shellfish Res. 7:19-23.

Burreson, E. M. \& L. M. Ragone Calvo. 1996. Epizootiology of Perkinsus marinus disease of oysters in the Chesapeake Bay with emphasis on data since 1985. J. Shellfish Res. 15(1):17-34.

Carnegie, R. \& E. M. Burreson. 2006. Status of the major oyster diseases in Virginia - 2005. A summary of the annual monitoring program. Virginia Institute of Marine Science Marine Resources Report. 14 pp.

Chai, A.-L., M. Homer, C.-F. Tsai \& P. Goulletquer. 1992. Evaluation of oyster sampling efficiency of patent tongs and an oyster dredge. N. Am. J. Fish. Manage. 12:825-832.

Cox, C. \& R. Mann. 1992. Temporal and spatial changes in fecundity of eastern oysters, Crassostrea virginica (Gmelin, 1791) in the James River, Virginia. J. Shellfish Res. 11:49-54.

Fegley, S. R. 2001. Demography and dynamics of hard clam populations. In: J. N. Kraeuter \& M. Castagna, editors. Biology of the Hard Clam. New York: Elsevier Science. pp. 383-422.

Ford, S. E. 1992. Avoiding the transmission of disease in commercial culture of molluscs, with special reference to Perkinsus marinus
(Dermo) and Haplosporidium nelsoni (MSX). J. Shellfish Res. 11:539-546.

Ford, S. E. \& H. H. Haskin. 1988. Management strategies for MSX (Haplosporidium nelsoni) disease in Eastern oysters. In: W.S. Fisher, editor. Disease processes in marine bivalve molluscs. American Fisheries Soc. Special Pub. 18. pp. 249-256.

Ford, S. D. \& M. R. Tripp. 1996. Diseases and defense mechanisms. In: V. S. Kennedy, R. I. E. Newell \& A. F. Eble, editors. The Eastern Oyster, Crassostrea virginica. College Park, Maryland: University of Maryland Sea Grant Press. pp. 581-660.

Hamrick, J. M. 1992. A three-dimensional environmental fluid dynamics computer code: theoretical and computational aspects. Virginia Institute of Marine Science, special report Applied Marine Science and Ocean Engineering No. 317. 64 pp.

Harding, J. M., R. Mann \& M. Southworth. 2008. Shell lengthat-age relationships in James River, Virginia oysters (Crassostrea virginica) collected four centuries apart. J. Shellfish Res. 27(In press).

Hargis, W. J., Jr. 1999. The evolution of the Chesapeake oyster reef system during the Holocene Epoch. In: M. W. Luckenbach, R. Mann \& J. A. Wesson, editors. Oyster reef habitat restoration: a synopsis of approaches. Gloucester Point, Virginia: Virginia Institute of Marine Science. pp. 5-24.

Hargis, W. J., Jr. \& D. S. Haven. 1988. The imperiled oyster industry of Virginia. A critical analysis with recommendations for restoration. Virginia Institute of Marine Science, special report Applied Marine Science of Ocean Engineering No. 290. 146 pp.

Hargis, W. J., Jr. \& D. S. Haven. 1995. The precarious state of the Chesapeake public oyster resource. In: P. Hill \& S. Nelson, editors. Proceedings of the 1994 Chesapeake Research Conference. Toward a sustainable coastal watershed: the Chesapeake experiment. Chesapeake Research Consortium Publication No. 149. pp. 559-584.

Hargis, W. J., Jr. \& D. S. Haven. 1999. Chesapeake oyster reefs, their importance, destruction and guidelines for restoring them. In: M. W. Luckenbach, R. Mann \& J. A. Wesson, editors. Oyster Reef habitat restoration: a synopsis and synthesis of approaches. Gloucester Point, Virginia: Virginia Institute of Marine Science. pp. 329-358.

Haven, D. S., J. P. Whitcomb \& P. Kendall. 1981. The present and potential productivity of the Baylor Grounds in Virginia. Virginia Institute of Marine Science, special report Applied Marine Science of Ocean Engineering No. 243. 154 pp.

Haven, D. S. \& J. P. Whitcomb. 1983. The origin and extent of oyster reefs in the James River, Virginia. J. Shellfish Res. 3:141-151.

Haven, D. S. \& L. W. Fritz. 1985. Setting of the American oyster, Crassostrea virginica in the James River, Virginia, USA: temporal and spatial distribution. Mar. Biol. 86:271-282.

HSRL. 2007. Report of the 2007 Stock Assessment Workshop (9th SAW) for the New Jersey Delaware Bay Oyster Beds. 62 pp.

HSRL. 2008. Report of the 2008 Stock Assessment Workshop (10th SAW) for the New Jersey Delaware Bay Oyster Beds. 62 pp.

Ingersoll, E. 1881. The oyster industry. In: The history and present condition of the fishery industries: Tenth Census of the United States, Department of the Interior, Washington, DC. 251 pp.

Kraeuter, J. N., S. Ford \& M. Cummings. 2007. Oyster growth analysis: a comparison of methods. J. Shellfish Res. 26:479-491. 
Luckenbach, M., R. Mann \& J. A. Wesson (eds). 1999. Oyster Reef habitat restoration: a synopsis of approaches. Virginia Institute of Marine Science, Gloucester Point. 366 pp.

Mann, R. 1988. Field studies of bivalve larvae at a frontal system in the James River, Virginia. Mar. Ecol. Prog. Ser. 50:29-44.

Mann, R. 2000. Restoring oyster reef communities in the Chesapeake Bay: A commentary. J. Shellfish Res. 19:335-340.

Mann, R. \& D. A. Evans. 1998. Estimation of oyster, Crassostrea virginica, standing stock, larval production and advective loss in relation to observed recruitment in the James River, Virginia. $J$. Shellfish Res. 17:239-254.

Mann, R. \& D. A. Evans. 2004. Site selection for oyster habitat rehabilitation in the Virginia portion of the Chesapeake Bay. $J$. Shellfish Res. 23:41-49.

Mann, R., F. Perkins, J.A. Wesson \& I.K. Bartol. 1996. Intertidal oyster reefs as a tool for estuarine rehabilitation and rejuvination of the Virginia oyster fishery. Final report submitted to Department of Environmental Quality, Virginia Coastal Resource Management Program, Richmond, Virginia.

Mann, R. \& E. N. Powell. 2007. Why oyster restoration goals in the Chesapeake Bay are not and probably cannot be achieved. $J$. Shellfish Res. 26:905-917.

Mann, R., M. Southworth, J. M. Harding \& J. E. Wesson. 2004. A comparison of dredge and patent tongs for estimation of oyster populations. J. Shellfish Res. 23:387-390.

Moore, H.F. 1910. Condition and extent of the oyster beds of James River, Virginia. Bureau of Fisheries, Doc \#729. Dept. of Commerce and Labor, Government of Printing Office, Washington, DC.

Powell, E. \& H. Cummings. 1985. Are molluscan maximum life spans determined by long-term cycles in benthic communities? Oecologica. 67:177-182.

Powell, E., K. Ashton-Alcox, J. Dobarro, M. Cummings \& S. Banta. 2002. The inherent efficiency of oyster dredges in survey mode. $J$. Shellfish Res. 21:691-695.

Powell, E. N. \& J. M. Klinck. 2007. Is oyster shell a sustainable estuarine resource? J. Shellfish Res. 26:181-194.

Powell, E. N., J. N. Kraeuter \& K. A. Ashton-Wilcox. 2006. How long does oyster shell last on an oyster reef? Estuar. Coast. Shelf Sci. 69:531-542.

Pritchard, D. W. 1952. Salinity distribution and circulation in the Chesapeake Bay estuarine system. J. Mar. Res. 11:106-123.

Pritchard, D. W. 1953. Distribution of oyster larvae in relation to hydrographic conditions. Proc. Gulf Caribb. Fish. Inst. 5:123-132.

Rheinhardt, R. D. \& R. Mann. 1990. Development of epibenthic fouling communities on a natural oyster bed in the James River, Virginia. Biofouling 2:13-25.

Ruzecki, E. P. \& W. J. Hargis, Jr. 1989. Interaction between circulation of the James River and transport of oyster larvae. In: B. Neilson, A.Y. Kuo \& J.M Brubaker, editors. Circulation patterns in estuaries. Clifton, New Jersey: Humana Press. pp. 273-278.

Scheltema, A. H. 1985. The aplacophoran family Prochaetodermatidae in the North American basin, including Chevroderma N.G. and Spathoderma N.G. (Mollusca; Chaetodermomorpha). Biol. Bull. 169:484-529.

Shen, J. \& A. Y. Kuo. 1999. Numerical investigation of an estuarine front and its associated eddy. J. Waterways, Ports. Coast. and Ocean Eng. 125:127-135.

Shen, J., J. D. Boon \& A. Y. Kuo. 1999. A modeling study of a tidal intrusion front and its impact on larval dispersion in the James River Estuary, Virginia. Estuaries 22:681-692.
Southworth, M., J. M. Harding \& R. Mann. 1999. The status of Virginia's public oyster resource 1998. Virginia Marine Resources Report No. 99-6. 37 pp.

Southworth, M., J. M. Harding \& R. Mann. 2000a. Management of oyster broodstock sanctuaries in the Great Wicomico and Piankatank Rivers, Virginia: optimal use of cultch to maximize settlement. Final report to Virginia Department of Environmental Quality, Coastal Resources Management Program. 12 pp.

Southworth, M., J. M. Harding \& R. Mann. 2000b. The status of Virginia's public oyster resource 1999. Virginia Marine Resources Report No. 2000-03. 38 pp.

Southworth, M., J. M. Harding \& R. Mann. 2001. The status of Virginia's public oyster resource 2000. Molluscan Ecology Program, Virginia Institute of Marine Science, Gloucester Point, Virginia. $56 \mathrm{pp}$

Southworth, M., J.M. Harding \& R. Mann. 2002. The status of Virginia's public oyster resource 2001. Molluscan Ecology Program, Virginia Institute of Marine Science, Gloucester Point, Virginia. $53 \mathrm{pp}$.

Southworth, M., J. M. Harding \& R. Mann. 2003. The status of Virginia's public oyster resource 2002. Molluscan Ecology Program, Virginia Institute of Marine Science, Gloucester Point, Virginia. $51 \mathrm{pp}$.

Southworth, M., J. M. Harding \& R. Mann. 2004. The status of Virginia's public oyster resource 2003. Molluscan ecology program, Virginia Institute of Marine Science, Gloucester Point, Virginia. $55 \mathrm{pp}$

Southworth, M., J. M. Harding \& R. Mann. 2005. The status of Virginia's public oyster resource 2004. Molluscan ecology program, Virginia Institute of Marine Science, Gloucester Point, Virginia. 51 pp.

Southworth, M., J. M. Harding \& R. Mann. 2006. The status of Virginia's public oyster resource 2005. Molluscan ecology program, Virginia Institute of Marine Science, Gloucester Point, Virginia. 49 pp.

Southworth, M., J. M. Harding \& R. Mann. 2007. The status of Virginia's public oyster resource 2006. Molluscan ecology program, Virginia Institute of Marine Science, Gloucester Point, Virginia. $49 \mathrm{pp}$.

Southworth, M. \& R. Mann. 1998. Oyster reef broodstock enhancement in the Great Wicomico River, Virginia. J. Shellfish Res. 17:11011114.

Southworth, M. \& R. Mann. 2004. Decadal scale changes in seasonal patterns of oyster recruitment in the Virginia sub estuaries of the Chesapeake Bay. J. Shellfish Res. 23:391-402.

Southworth, M., R. Mann, J. M. Harding \& J. A. Wesson. 2008. Multiyear recruitment patterns of oysters (Crassostrea virginica) on constructed reefs in the Chesapeake Bay. J. Shellfish Res. 27: 1053.

Volstad, J. H., J. Dew \& M. Tarnowski. 2008. Estimation of annual mortality rates for eastern oysters (Crassostrea virginica) in Chesapeake Bay based on box counts and application of those rates to project population growth of $C$. virginica and C. ariakensis. J. Shellfish Res. 27:525-533.

Wesson, J. A., R. Mann \& M. Luckenbach. 1999. Oyster restoration efforts in Virginia. In: M.W. Luckenbach, R. Mann \& J.A. Wesson, editors. Oyster reef habitat restoration: a synopsis of approaches. Gloucester Point: Virginia Institute of Marine Science. pp 117-130.

Wood, L. \& W. J. Hargis, Jr. 1971. Transport of bivalve larvae in a tidal estuary. In: D. J. Crisp, editor. Fourth Eur. Mar. Biol. Symp. Cambridge: Cambridge University Press. pp. 21-44. 